\title{
COMBINING LENS DISTORTION AND DEPLETION TO MAP THE MASS DISTRIBUTION OF A1689 ${ }^{1}$
}

\author{
KeIICHI Umetsu ${ }^{2,3}$ and Tom Broadhurst ${ }^{4}$ \\ Received 2007 December 20; accepted 2008 April 24
}

\begin{abstract}
We derive a projected 2D mass map of the well-studied galaxy cluster A1689 based on an entropy-regularized maximum likelihood combination of the lens magnification and distortion of red background galaxies registered in deep Subaru images. The method is not restricted to the weak regime but applies to the whole area outside the tangential critical curve, where nonlinearity between the surface mass density and the observables extends to a radius of a few arcminutes. The known strong-lensing information is also readily incorporated in this approach, represented as a central pixel with a mean surface density close to the critical value. We also utilize the distortion measurements to locally downweight the intrinsic clustering noise, which otherwise perturbs the depletion signal. The projected mass profile continuously steepens with radius and is well fitted by the Navarro-Frenk-White model, but with a surprisingly large concentration $c_{\mathrm{vir}}=13.4_{-3.3}^{+5.3}$, lying far from the predicted value of $c_{\mathrm{vir}} \sim 5$, corresponding to the measured virial mass, $M_{\text {vir }}=(2.1 \pm 0.2) \times 10^{15} M_{\odot}$, posing a challenge to the standard assumptions defining the $\Lambda C D M$ model. We examine the consistency of our results with estimates derived with the standard weak-lensing estimators and by comparison with the inner mass profile obtained from strong lensing. All the reconstructions tested here imply a virial mass in the range $M_{\mathrm{vir}}=(1.5-2.1) \times 10^{15} M_{\odot}$, and the combined ACS and Subaru 2D mass reconstruction yields a tight constraint on the concentration parameter, $c_{\mathrm{vir}}=12.7 \pm 1 \pm 2.8\left(c_{200} \sim 10\right)$, improving upon the statistical accuracy of our earlier 1D analysis. Importantly, our best-fitting profile properly reproduces the observed Einstein radius of $45^{\prime \prime}\left(z_{S}=1\right)$, in contrast to other weak-lensing work, reporting lower concentration profiles, which underestimate the observed Einstein radius.
\end{abstract}

Subject headings: cosmology: observations — dark matter — galaxies: clusters: individual (A1689) — gravitational lensing

Online material: color figures

\section{INTRODUCTION}

Weak gravitational lensing of background galaxies provides a unique, direct way to study the mass distribution of galaxy clusters (Bartelmann \& Schneider 2001) via the systematic shape distortion of background galaxies (Tyson et al. 1990; Kaiser \& Squires 1993; Schneider \& Seitz 1995; Umetsu et al. 1999) and also to a lesser extent by the magnification of the background (Broadhurst et al. 1995; Taylor et al. 1998). We have examined both these effects in our earlier work on A1689 (Broadhurst et al. 2005a, hereafter B05a), where we found good consistency between the magnitude of the radial depletion of background red galaxies caused by lens magnification and the weak-lensing distortion profile of the same background galaxy population, which we then combined to derive an improved mass profile.

A limitation of the magnification technique is the intrinsic clustering of the background, which for red galaxies is mainly in the form of localized groups of modest angular size in the background field. In principle, with redshift information, sharp overdensities can be isolated in redshift and downweighted, or with sufficiently large number of redshifts, the shift of the magnified luminosity function can be utilized independently of density fluctuations (Broadhurst et al. 1995; Zhang \& Pen 2005). The combination of all lensing-related effects is of course desirable,

\footnotetext{
1 Based in part on data collected at the Subaru Telescope, which is operated by the National Astronomical Society of Japan.

2 Institute of Astronomy and Astrophysics, Academia Sinica, P.O. Box 23-141, Taipei 106, Taiwan.

3 Leung Center for Cosmology and Particle Astrophysics, National Taiwan University, Taipei 106, Taiwan.

4 School of Physics and Astronomy, Tel Aviv University, Israel.
}

leading to the derivation of the highest precision feasible when constructing mass maps and density profiles. Furthermore, because lensing effects depend on distance, the cosmological redshift distance relation may be constrained via the geometric scaling of the lensing signal with redshift (e.g., Taylor et al. 2007; Medezinski et al. 2007).

Advances in the quality of imaging encourage a closer examination of the empirical effects of lensing and the development of more comprehensive techniques to extract reliable highresolution information. From space, deep multicolor images of massive clusters can be used to identify many sets of multiple images per cluster (Broadhurst et al. 2005b, hereafter B05b; Gavazzi et al. 2003; Kneib et al. 2003; Sand et al. 2004; Smith et al. 2005; Bradač et al. 2006). From the ground, the stable prime-focus wide-field cameras of Subaru and CFHT are producing data of sufficient quality to examine weak-lensing distortions over a wide range of radius. More recently, wide-field near-IR cameras, such as MOIRCS on Subaru, WIRCAM on CFHT, OMEGA2000 on Calar Alto, and WFCAM on UKIRT, may help improve the accuracy of photometric redshifts for many faint galaxies. However, it is still the case that no set of deep high-quality wide-field images exists for any massive cluster with full optical-IR coverage, despite all the progress of field surveys.

A further motivation for pursuing accurate lensing maps is the increased precision of model predictions for statistical properties of cluster-sized mass halos in the standard $\Lambda$ cold dark matter $(\Lambda C D M)$ model. Many of the free parameters of this model now rest on a firm empirical foundation with relatively tight constraints on the index and normalization of the power spectrum of density perturbation and the background cosmological model 
(e.g., Spergel et al. 2003, 2007; Tegmark et al. 2004). In this context $\mathrm{N}$-body simulations have become impressively comprehensive, in particular, the recent Millenium simulation (Springel et al. 2005) which simulates a huge volume of $500 \mathrm{Mpc}^{-1}$, and has been used to predict the mass function and evolution of nearly 100,000 group and cluster-sized CDM halos. This model is tightly defined and hence amenable to comparisons with the real universe, particularly for the case of clusters where baryons, which are usually omitted from large-scale simulation, are not expected to have a significant impact on the shape of gravitational potential of a cluster since the high temperature of the cluster gas prevents efficient cooling, and hence the majority of baryons simply trace the gravitational potential of the dominant dark matter. Accurate $N$-body simulations based on the $\Lambda \mathrm{CDM}$ scenario predict a relatively shallow, low-concentration mass profile for massive cluster halos, where the logarithmic gradient flattens continuously toward the center of mass (Navarro et al. 1997) with a flatter central slope than a purely isothermal body interior to the inner characteristic radius, $r_{s} \lesssim 100-200 \mathrm{kpc} h^{-1}$. A useful index of the degree of concentration, $c_{\mathrm{vir}}$, compares the virial radius, $r_{\mathrm{vir}}$, to $r_{s}$ of the Navarro-Frenk-White (NFW) profile, $c_{\mathrm{vir}} \equiv r_{\mathrm{vir}} / r_{s}$. This prediction for the CDM halo $c_{\mathrm{vir}}-M_{\mathrm{vir}}$ relation has been established thoroughly with high-resolution simulations (e.g., Navarro et al. 1997; Bullock et al. 2001; Wechsler et al. 2002; Neto et al. 2007) with some intrinsic variation related to the individual assembly history of a cluster (e.g., Jing \& Suto 2000; Tasitsiomi et al. 2004; Hennawi et al. 2007). In particular, the detailed $N$-body millennium simulation (Springel et al. 2005) predicts a simple relationship between the halo mass and concentration parameter for halo masses in the range of galaxy groups to massive clusters, as quantified by Neto et al. (2007), who found the expected median value for cluster-sized halos of $M_{\mathrm{vir}} \sim$ $10^{15} M_{\odot}$ to be $c_{\mathrm{vir}} \sim 5$ at $z=0\left(c_{200} \sim 4\right)$, with a spread of the order of $\Delta \log c_{\mathrm{vir}}=0.1$. (See Johnston et al. [2007] for a good summary of the state of the art in halo concentrations based on Wechsler et al. [2006] and Neto et al. [2007].)

In this paper we explore further methods designed to achieve the maximum possible lensing precision by combining all lensing information for A1689. This cluster is among the most massive clusters with the largest known Einstein radius $\left(\sim 50^{\prime \prime}\right)$, and is one of the best-studied clusters for lensing work (Tyson et al. 1990; Tyson \& Fisher 1995; Taylor et al. 1998; King et al. 2002; Bardeau et al. 2005, 2007; B05a; B05b; Oguri et al. 2005; Halkola et al. 2006; Limousin et al. 2007; Medezinski et al. 2007; Umetsu et al. 2007; Okura et al. 2008), located at a moderately low redshift of $z=0.183$. In B05a we developed a "model-independent" method $^{5}$ for reconstructing the cluster mass profile using azimuthally averaged weak-lensing shape distortion and magnification bias measurements, in the wide-field, Subaru images. This together with many multiple images identified in deep Hubble Space Telescope (HST) Advanced Camera for Surveys (ACS) imaging defined a detailed lensing-based cluster mass profile out to the cluster virial radius $\left(r \lesssim 2 h^{-1} \mathrm{Mpc}\right)$. The combined strongand weak-lensing mass profile is well fitted by an NFW profile (Navarro et al. 1997) with a high concentration of $c_{\mathrm{vir}} \sim 13.7$, which is significantly larger than theoretically expected $\left(c_{\mathrm{vir}} \sim 5\right)$ for the standard $\Lambda$ CDM model (Bullock et al. 2001; Neto et al. 2007), although the degree of concentration is still controversial (B05a; Medezinski et al. 2007; Limousin et al. 2007). Such a

\footnotetext{
5 We remind the reader that model dependence is unavoidable to some extent in scientific analysis. In this work we define the term "model independent" to refer to those methods without prior assumptions about the functional form of the lensing profiles and distributions.
}

high concentration is also seen in other massive clusters from careful lensing work, such as MS 2137-23 ( $c_{200} \simeq 12$; Gavazzi et al. 2003) and CL $0024+1654\left(c_{200} \simeq 22\right.$; Kneib et al. 2003). These results could raise serious questions regarding the basic assumptions behind the $\Lambda \mathrm{CDM}$ model. If clusters collapse earlier than predicted, then it is expected that denser and hence more concentrated halos will develop in the context of CDM (Wechsler et al. 2002). On the other hand, it has been argued that part of this discrepancy from lensing observations could be reconciled by observational effects such as triaxiality of CDM halos (Oguri et al. 2005; Hennawi et al. 2007; Sereno 2007; Corless \& King 2007), and the projection of structure along the line of sight (e.g., King \& Corless 2007), both of which boost the projected surface mass density and hence the lensing signal. Such observational biases in the lensing-based concentration parameter have been explored in detail by Hennawi et al. (2007) on the basis of $\mathrm{N}$-body simulations, indicating a positive bias of $\sim 30 \%$ in the halo concentration derived from $2 \mathrm{D}$ lensing measurements. Although A1689 is a very round shaped cluster with evidence of only modest substructure (Teague et al. 1990; Girardi et al. 1997; Andersson \& Madejski 2004; Czoske 2004; B05b), such a chance alignment of structure could be a potential source of high concentrations. Furthermore, for a reliable measurement of the cluster mass profile, systematic errors inherent in the lensing measurements, such as the uncertainty in the background redshift distribution and the dilution effect on the lensing signal due to contamination by cluster members (B05a; Medezinski et al. 2007), need to be taken into account.

The paper is organized as follows. We briefly summarize in $\S 2$ the basis of cluster weak lensing. In $\S 3$ we describe the observational data and the background sample selection for the weaklensing analysis; we then summarize our joint weak-lensing analysis of shape distortion and magnification bias data. In $\S 4$ we present a method for reconstructing the two-dimensional mass distribution of A1689 from combined weak-lensing shape distortion and magnification bias measurements. In $\S 5$ we derive mass profiles of A1689 from weak-lensing data using three different methods, and compare resulting mass profiles; we also combine our weak-lensing mass profiles with strong-lensing constraints from previous studies to test the CDM paradigm; then, we assess carefully various sources of potential systematic error in the halo concentration parameter derived from the lensing observations. Finally, summary and discussions are given in $\S 6$.

Throughout this paper, we use the $\mathrm{AB}$ magnitude system, and adopt a concordance $\Lambda$ CDM cosmology with $\left(\Omega_{m 0}=0.3, \Omega_{\Lambda 0}=\right.$ $0.7, h=0.7)$. In this cosmology $1^{\prime}$ corresponds to the physical scale $129 \mathrm{kpc} h^{-1}$ for this cluster. The reference center of our analysis is fixed at the center of the cD galaxy: R.A. $=13^{\mathrm{h}} 11^{\mathrm{m}} 29.52^{\mathrm{s}}$, decl. $=-01^{\circ} 20^{\prime} 27.59^{\prime \prime}(\mathrm{J} 2000.0)$.

\section{CLUSTER WEAK LENSING}

Weak gravitational lensing is responsible for the weak shape distortion and magnification of the images of background sources due to the gravitational field of intervening foreground clusters of galaxies and large-scale structures in the universe. The deformation of the image can be described by the $2 \times 2$ Jacobian matrix $\mathcal{A}_{\alpha \beta}(\alpha, \beta=1,2)$ of the lens mapping. The Jacobian $\mathcal{A}_{\alpha \beta}$ is real and symmetric, so that it can be decomposed as

$$
\begin{aligned}
\mathcal{A}_{\alpha \beta} & =(1-\kappa) \delta_{\alpha \beta}-\Gamma_{\alpha \beta}, \\
\Gamma_{\alpha \beta} & =\left(\begin{array}{cc}
+\gamma_{1} & \gamma_{2} \\
\gamma_{2} & -\gamma_{1}
\end{array}\right),
\end{aligned}
$$


where $\delta_{\alpha \beta}$ is Kronecker's delta, $\Gamma_{\alpha \beta}$ is the trace-free, symmetric shear matrix with $\gamma_{\alpha}$ being the components of spin-2 complex gravitational shear $\gamma \equiv \gamma_{1}+i \gamma_{2}$, describing the anisotropic shape distortion, and $\kappa$ is the lensing convergence responsible for the trace part of the Jacobian matrix, describing the isotropic area distortion. In the weak-lensing limit where $\kappa,|\gamma| \ll 1, \Gamma_{\alpha \beta}$ induces a quadrupole anisotropy of the background image, which can be observed from ellipticities of background galaxy images. The flux magnification due to gravitational lensing is given by the inverse Jacobian determinant,

$$
\mu=\frac{1}{\operatorname{det} \mathcal{A}}=\frac{1}{(1-\kappa)^{2}-|\gamma|^{2}},
$$

where we assume subcritical lensing, i.e., $\operatorname{det} \mathcal{A}(\boldsymbol{\theta})>0$.

The lensing convergence is expressed as a line-of-sight projection of the matter density contrast out to the source plane $(s)$ weighted by a certain combination $g$ of comoving angular diameter distances (e.g., Jain et al. 2000),

$$
\kappa=\frac{3 H_{0}^{2} \Omega_{m}}{2 c^{2}} \int_{0}^{\chi_{s}} d \chi g\left(\chi, \chi_{s}\right) \frac{\delta}{a} \equiv \int d \Sigma_{m} \Sigma_{\text {crit }}^{-1},
$$

where $a$ is the cosmic scale factor, $\chi$ is the comoving distance, $\Sigma_{m}$ is the surface mass density of matter, $\Sigma_{m}=\int_{0}^{\chi_{s}} d \chi a\left(\rho_{m}-\bar{\rho}\right)$, with respect to the cosmic mean density $\bar{\rho}$, and $\Sigma_{\text {crit }}$ is the critical surface mass density for gravitational lensing,

$$
\Sigma_{\mathrm{crit}}=\frac{c^{2}}{4 \pi G} \frac{D_{s}}{D_{d} D_{d s}}
$$

with $D_{s}, D_{d}$, and $D_{d s}$ being the angular diameter distances from the observer to the source, from the observer to the deflecting lens, and from the lens to the source, respectively. For a fixed background cosmology and a lens redshift $z_{d}, \Sigma_{\text {crit }}$ is a function of background source redshift $z_{s}$. For a given mass distribution $\Sigma_{m}(\boldsymbol{\theta})$, the lensing signal is proportional to the angular diameter distance ratio, $D_{d s} / D_{s}$.

In the present weak-lensing study we aim to reconstruct the dimensionless surface mass density $\kappa$ from weak-lensing distortion and magnification data. To do this, we utilize the relation between the gradients of $\kappa$ and $\gamma$ (Kaiser 1995; Crittenden et al. 2002),

$$
\triangle \kappa(\boldsymbol{\theta})=\partial^{\alpha} \partial^{\beta} \Gamma_{\alpha \beta}(\boldsymbol{\theta})=2 \hat{\mathcal{D}}^{*} \gamma(\boldsymbol{\theta}),
$$

where $\hat{\mathcal{D}}$ is the complex differential operator $\hat{\mathcal{D}}=\left(\partial_{1}^{2}-\partial_{2}^{2}\right) / 2+$ $i \partial_{1} \partial_{2}$. The Green's function for the two-dimensional Poisson equation is $\triangle^{-1}\left(\boldsymbol{\theta}, \boldsymbol{\theta}^{\prime}\right)=\ln \left|\boldsymbol{\theta}-\boldsymbol{\theta}^{\prime}\right| /(2 \pi)$, so that equation (6) can be solved to yield the following nonlocal relation between $\kappa$ and $\gamma$ (Kaiser \& Squires 1993):

$$
\kappa(\boldsymbol{\theta})=\frac{1}{\pi} \int d^{2} \theta^{\prime} D^{*}\left(\boldsymbol{\theta}-\boldsymbol{\theta}^{\prime}\right) \gamma\left(\boldsymbol{\theta}^{\prime}\right),
$$

where $D(\boldsymbol{\theta})$ is the complex kernel defined as

$$
D(\boldsymbol{\theta})=\frac{\theta_{2}^{2}-\theta_{1}^{2}-2 i \theta_{1} \theta_{2}}{|\theta|^{4}}
$$

Similarly, the spin-2 shear field can be expressed in terms of the lensing convergence as

$$
\gamma(\boldsymbol{\theta})=\frac{1}{\pi} \int d^{2} \theta^{\prime} D\left(\boldsymbol{\theta}-\boldsymbol{\theta}^{\prime}\right) \kappa\left(\boldsymbol{\theta}^{\prime}\right) .
$$

Note that adding a constant mass sheet to $\kappa$ in equation (9) does not change the shear field $\gamma(\boldsymbol{\theta})$ which is observable in the weaklensing limit, leading to the so-called mass-sheet degeneracy based solely on shape-distortion measurements (e.g., Bartelmann \& Schneider 2001; Umetsu et al. 1999). In general, the observable quantity is not the gravitational shear $\gamma$ but the reduced shear,

$$
g=\frac{\gamma}{1-\kappa}
$$

in the subcritical regime where $\operatorname{det} \mathcal{A}>0$ (or $1 / g^{*}$ in the negative parity region with $\operatorname{det} \mathcal{A}<0$ ). We see that the reduced shear $g$ is invariant under the following global transformation:

$$
\kappa(\boldsymbol{\theta}) \rightarrow \lambda \kappa(\boldsymbol{\theta})+1-\lambda, \quad \gamma(\boldsymbol{\theta}) \rightarrow \lambda \gamma(\boldsymbol{\theta}),
$$

with an arbitrary scalar constant $\lambda \neq 0$ (Schneider $\&$ Seitz 1995). This transformation is equivalent to scaling the Jacobian matrix $\mathcal{A}(\boldsymbol{\theta})$ with $\lambda, \mathcal{A}(\boldsymbol{\theta}) \rightarrow \lambda \mathcal{A}(\boldsymbol{\theta})$. This mass-sheet degeneracy can be unambiguously broken by measuring the magnification effects, because the magnification $\mu$ transforms under the invariance transformation (11) as

$$
\mu(\boldsymbol{\theta}) \rightarrow \lambda^{2} \mu(\boldsymbol{\theta})
$$

\section{DATA ANALYSIS}

In this section we present a full technical description of our weak-lensing distortion and magnification analyses on A1689 based on the Subaru images, which were analyzed in our earlier work of B05a and Medezinski et al. (2007). This work is also based on the same weak-lensing shape and magnification measurements as used in B05a. We note that Medezinski et al. (2007) used a slightly different analysis pipeline so as to include the shape measurements of bright cluster members and noisier objects (as well as better resolved red background galaxies), optimizing for the weak-lensing dilution analysis including the measurements of cluster light and cluster luminosity function where the completeness is crucial; Medezinski et al. (2007) included a blue background population in the weak-lensing shape analysis, in addition to the red background population which B05a and this work are based on. The magnification information, on the other hand, was not taken into account in Medezinski et al. (2007).

In B05a we simply assumed that the mean redshift of the red background galaxies is $z_{s}=1$. In the present work we improve the accuracy of determination of the cluster mass and concentration parameters, by taking into account the redshift distribution of red background galaxies examined by Medezinski et al. (2007) based on the multicolor photometry of Capak et al. (2004) in the HDF-N (see $\S 3.3)$.

\subsection{Subaru Data and Photometry}

For our weak-lensing analysis we used Subaru Suprime-Cam imaging data of A1689 in $V$ and SDSS $i^{\prime}$ retrieved from the Subaru archive, SMOKA (see B05a; Medezinski et al. 2007 for more details). The FWHM in the co-added mosaic image is $0.82^{\prime \prime}$ in $V$ and $0.88^{\prime \prime}$ in $i^{\prime}$ with $0.202^{\prime \prime}$ pixel $^{-1}$, covering a field of $\approx 30^{\prime} \times 25^{\prime}$.

Photometry is based on a combined $V+i^{\prime}$ image with SExtractor (Bertin \& Arnouts 1996), where the $i^{\prime}$ image is used as the source detection image. We adopt the following key configuration parameters in SExtractor: DETECT_MINAREA $=5$, DETECT_THRESH $=$ ANALYSIS_THRESH $=3$. The limiting magnitudes are obtained as $V=26.5$ and $i^{\prime}=26.0$ for a $3 \sigma$ 
detection within a $2^{\prime \prime}$ aperture. A careful background selection is critical for a weak-lensing analysis so that unlensed cluster members and foreground galaxies do not dilute the true lensing signal of the background (see B05a; Medezinski et al. 2007). We identify an E/S0 sequence of cluster galaxies in the colormagnitude $(\mathrm{CM})$ diagram, which can be defined by the linear CM relation: $\left(V-i^{\prime}\right)_{E / S}=-0.02094 i^{\prime}+1.255$ (B05a; see Fig. 1 of Medezinski et al. 2007 for the CM diagram). For the number counts to measure lensing magnification, we define a sample of galaxies that are redder than the cluster sequence by $\left(V-i^{\prime}\right)>$ 1.0 and $20<i^{\prime}<25.5$, which yields a total of $N_{\mu}=8907$ galaxies, or the mean surface number density of $\bar{n}_{\mu}=12.0 \mathrm{arcmin}^{-2}$. For the magnification bias analysis, a conservative magnitude limit of $i^{\prime}<25.5$ is adopted to avoid incompleteness.

\subsection{Weak-Lensing Distortion Analysis}

We use the IMCAT package developed by N. Kaiser ${ }^{6}$ to perform object detection, photometry and shape measurements, following the formalism outlined in Kaiser et al. (1995; hereafter KSB95). We have modified the method somewhat following the procedures described in Erben et al. (2001). We used the same analysis pipeline as in B05a, Umetsu et al. (2007), and Okabe \& Umetsu (2008).

\subsubsection{Object Detection}

Objects are first detected as local peaks in the image by using the IMCAT hierarchical peak-finding algorithm hf indpeaks which for each object yields object parameters such as a peak position, an estimate of the object size $\left(r_{g}\right)$, the significance of the peak detection $(\nu)$. The local sky level and its gradient are measured around each object from the mode of pixel values on a circular annulus defined by inner and outer radii of $16 r_{g}$ and $32 r_{g}$ (see Clowe et al. 2000). In order to avoid contamination in the background estimation by bright neighboring stars and/or foreground galaxies, all pixels within $3 r_{g}$ of another object are excluded from the mode calculation. Total fluxes and half-light radii $\left(r_{h}\right)$ are then measured on sky-subtracted images using a circular aperture of radius $3 \sqrt{2} r_{g}$ from the object center. Any pixels within $2.5 r_{g}$ of another object are excluded from the aperture. The aperture $i^{\prime}$-magnitude is then calculated from the measured total flux and a zero-point magnitude. Any objects with positional differences between the peak location and the weighted centroid greater than $d=0.4$ pixels are excluded from the catalog.

Finally, bad objects such as spikes, saturated stars, and noisy detections must be removed from the weak-lensing object catalog. We removed from our object catalog (1) extremely large objects with $r_{g}>10$ pixels, (2) objects with low detection significance, $\nu<7$, (3) objects with large raw ellipticities, $|e|>$ 0.5 , (4) noisy detections with unphysical negative fluxes, and (5) objects containing more than 10 bad pixels, nbad $>10$. This selection procedure yields an object catalog with $N=62,384$ $\left(82.6 \mathrm{arcmin}^{-2}\right)$.

\subsubsection{Weak-Lensing Distortion Measurements}

To obtain an estimate of the reduced shear, $g_{\alpha}=\gamma_{\alpha} /(1-\kappa)$ ( $\alpha=1$ and 2), we measure the image ellipticity $e_{\alpha}=\left\{Q_{11}-\right.$ $\left.Q_{22}, Q_{12}\right\} /\left(Q_{11}+Q_{22}\right)$ from the weighted quadrupole moments of the surface brightness of individual galaxies defined in the above catalog,

$$
Q_{\alpha \beta}=\int d^{2} \theta W(\theta) \theta_{\alpha} \theta_{\beta} I(\boldsymbol{\theta}), \quad(\alpha, \beta=1,2),
$$

\footnotetext{
${ }^{6}$ See http://www.ifa.hawaii.edu/ kaiser/imcat.
}

where $I(\boldsymbol{\theta})$ is the surface brightness distribution of an object and $W(\theta)$ is a Gaussian window function matched to the size of the object.

First, the PSF anisotropy needs to be corrected using the star images as references:

$$
e_{\alpha}^{\prime}=e_{\alpha}-P_{\mathrm{sm}}^{\alpha \beta} q_{\beta}^{*}
$$

where $P_{\mathrm{sm}}$ is the smear polarizability tensor being close to diagonal, and $q_{\alpha}^{*}=\left(P_{\mathrm{sm}}^{*}\right)_{\alpha \beta}^{-1} e_{*}^{\beta}$ is the stellar anisotropy kernel. We select bright, unsaturated foreground stars of $20 \lesssim i^{\prime} \lesssim 22.5$ identified in a branch of the half-light radius $\left(r_{h}\right)$ versus magnitude $\left(i^{\prime}\right)$ diagram to measure $q_{\alpha}^{*}$. In order to obtain a smooth map of $q_{\alpha}^{*}$ which is used in equation (14), we divided the coadded mosaic image of $9 \mathrm{~K} \times 7.4 \mathrm{~K}$ pixels into $5 \times 4$ blocks, each with $1.8 \mathrm{~K} \times 1.85 \mathrm{~K}$ pixels. The block length is based on the typical coherent scale of PSF anisotropy patterns. In this way the PSF anisotropy in individual blocks can be well described by fairly low order polynomials. We then fitted the $q^{*}$ in each block independently with second-order bipolynomials, $q_{*}^{\alpha}(\boldsymbol{\theta})$, in conjunction with iterative $\sigma$-clipping rejection on each component of the residual: $\delta e^{*}=e_{\alpha}^{*}-\left(P_{\mathrm{sm}}^{*}\right)^{\alpha \beta} q_{\beta}^{*}(\boldsymbol{\theta})$. The final stellar sample consists of 540 stars (i.e., $N_{*} \sim 30$ stars per block), or the mean surface number density of $\bar{n}_{*}=0.72 \mathrm{arcmin}^{-2}$. It is worth noting that the mean stellar ellipticity before correction is $\left(\bar{e}_{1}^{*}, \bar{e}_{2}^{*}\right) \simeq$ $(-0.013,-0.018)$ over the data field, while the residual $e_{\alpha}^{*}$ after correction is reduced to $\bar{e}_{1}^{* \text { res }}=(0.47 \pm 1.32) \times 10^{-4}, \bar{e}_{2}^{* \text { res }}=$ $(0.54 \pm 0.94) \times 10^{-4}$. The mean offset from the null expectation is reduced down to $\left|\bar{e}^{\text {*res }}\right|=(0.71 \pm 1.12) \times 10^{-4}$. On the other hand, the rms value of stellar ellipticities, $\sigma_{e^{*}} \equiv\left\langle\left|e^{*}\right|^{2}\right\rangle^{1 / 2}$, is reduced from $2.64 \%$ to $0.38 \%$ when applying the anisotropic PSF correction. We show in Figure 1 the quadrupole PSF anisotropy field as measured from stellar ellipticities before and after the anisotropic PSF correction. Figure 2 shows the distribution of stellar ellipticity components before and after the PSF anisotropy correction. From the rest of the object catalog, we select objects with $\bar{r}_{h *} \lesssim r_{h} \lesssim 10$ pixels as an $i^{\prime}$-selected weak-lensing galaxy sample, where $\bar{r}_{h *} \approx 2.4$ pixels is the median value of stellar half-light radii, corresponding to half the median width of circularized PSF over the data field. An apparent magnitude cutoff of $20 \lesssim i^{\prime} \lesssim 26$ is also made to remove from the weak-lensing galaxy sample bright foreground/cluster galaxies and very faint galaxies with noisy shape measurements.

Second, we need to correct image ellipticities for the isotropic smearing effect caused by atmospheric seeing and the window function used for the shape measurements. The preseeing reduced shear $g_{\alpha}$ can be estimated from

$$
g_{\alpha}=\left(P_{g}^{-1}\right)_{\alpha \beta} e_{\beta}^{\prime},
$$

with the preseeing shear polarizability tensor $P_{\alpha \beta}^{g}$. We follow the procedure described in Erben et al. (2001) to measure $P^{g}$. We adopt the scalar correction scheme, namely (Erben et al. 2001; Hoekstra et al. 1998; Hudson et al. 1998; Okabe \& Umetsu 2008),

$$
\left(P_{g}\right)_{\alpha \beta}=\frac{1}{2} \operatorname{tr}\left(P_{g}\right) \delta_{\alpha \beta} \equiv P_{g}^{s} \delta_{\alpha \beta} .
$$

In order to suppress artificial effects due to the noisy $P_{g}^{s}$ estimated for individual galaxies, we apply filtering to raw $P_{g}^{s}$ measurements. First, we discard those noisy objects which have negative raw $P_{g}^{s}$ values. Second, we compute for each object a median value of $P_{g}^{s}$ among $N$-neighbors in the size $r_{g}$ and 


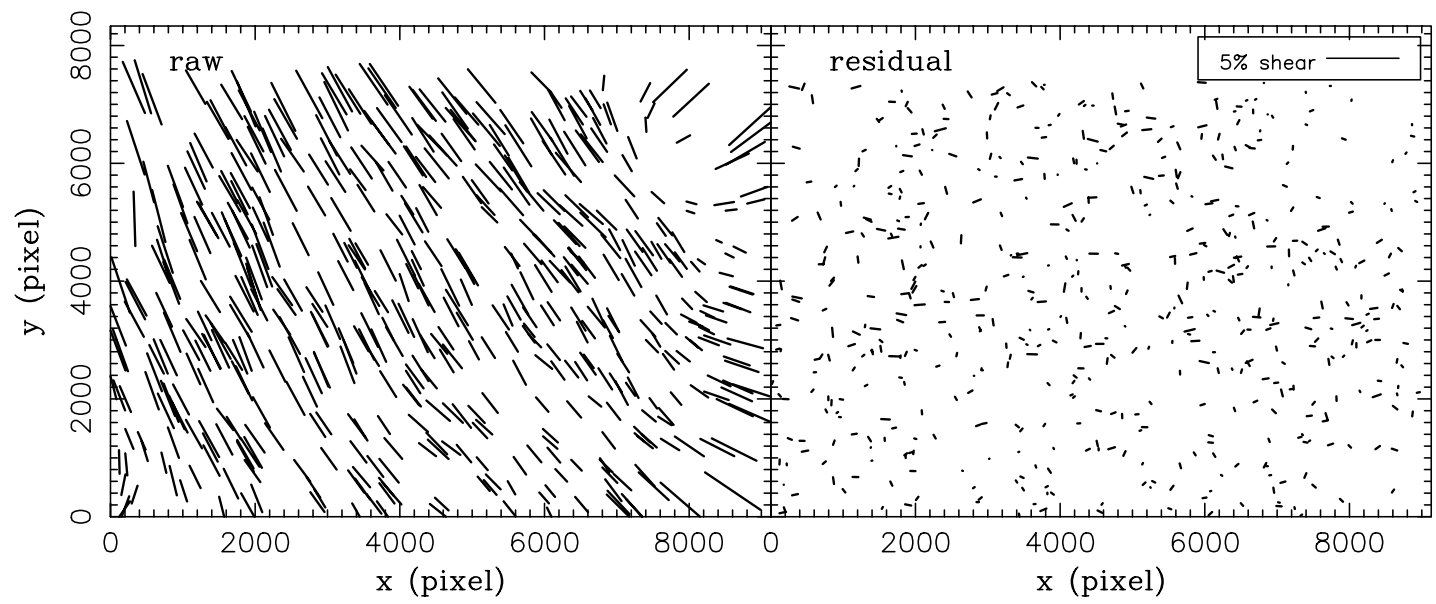

FIG. 1.-Quadrupole PSF anisotropy field as measured from stellar ellipticities before and after the PSF anisotropy correction. Left: Raw ellipticity field of stellar objects; right: residual ellipticity field after the PSF anisotropy correction. The orientation of the sticks indicates the position angle of the major axis of stellar ellipticity, whereas the length is proportional to the modulus of stellar ellipticity. A stick with a length of $5 \%$ ellipticity is indicated in the top right of the right panel.

magnitude $i^{\prime}$-plane to define object parameter space: for each object, $N$ neighbors are identified in the size $\left(r_{g}\right)$ and magnitude $\left(i^{\prime}\right)$ plane; the median value of $P_{g}^{s}$ is used as the smoothed $P_{g}^{s}$ for the object, $\left\langle P_{g}^{s}\right\rangle$, and the variance $\sigma_{g}^{2}$ of $g=g_{1}+i g_{2}$ is calculated using equation (15). The dispersion $\sigma_{g}$ is used as an rms error of the shear estimate for individual galaxies. We take $N=30$. After filtering noisy $P_{g}^{s}$ measurements, the minimum value of $\left\langle P_{g}^{s}\right\rangle$ is $\approx 0.035$. Figure 3 shows the averaged $\left\langle P_{g}^{s}\right\rangle$ as a function of object size $r_{g}$ for the $i^{\prime}$-selected weak-lensing galaxy sample. The mean of $\left\langle P_{g}^{s}\right\rangle$ over all galaxies in the sample is obtained as 0.307 , mostly weighted by galaxies with $r_{g}=2-3$ pixels. The mean variance $\bar{\sigma}_{g}^{2}$ over the galaxy sample is obtained as $\simeq 0.152$, or $\left(\overline{\sigma_{g}^{2}}\right)^{1 / 2} \approx 0.39$. Finally, we use the following estimator for the reduced shear: $g_{\alpha}=e_{\alpha}^{\prime} /\left\langle P_{g}^{s}\right\rangle$. The final $i^{\prime}$-selected galaxy sample contains 30,369 galaxies, or $\bar{n}_{g} \simeq 40.3 \mathrm{arcmin}^{-2}$.

\subsection{Red Background Selection}

As demonstrated by B05a and Medezinski et al. (2007), it is crucial in the weak-lensing analysis to make a secure selection of background galaxies in order to minimize contamination by cluster/foreground galaxies and hence to make an accurate determination of the cluster mass; otherwise, dilution of the distortion signal results from the inclusion of unlensed cluster galaxies, particularly at small radius where the cluster is relatively dense. This dilution effect is simply to reduce the strength of the lensing signal when averaged over a local ensemble of galaxies, in pro-

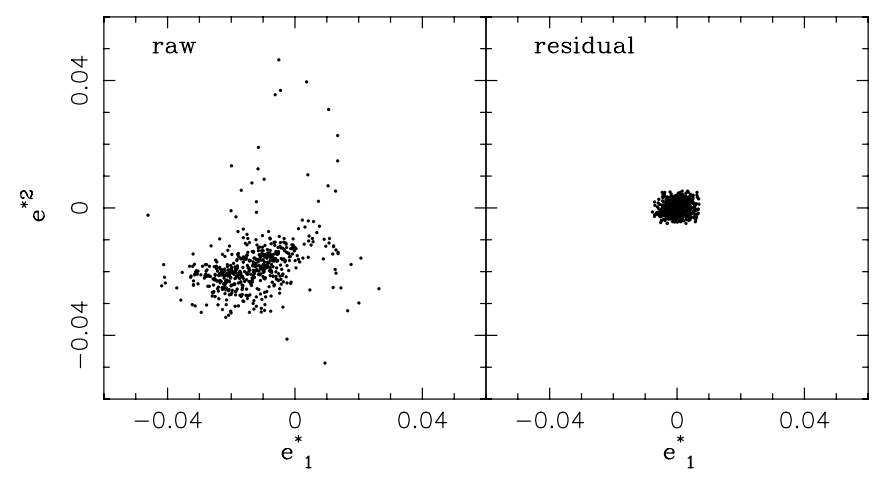

FIG. 2.- Stellar ellipticity distributions before and after the PSF anisotropy correction. Left: Raw ellipticity components $\left(e_{1}^{*}, e_{2}^{*}\right)$ of stellar objects; right: residual ellipticity components $\left(\delta e_{1}^{*}, \delta e_{2}^{*}\right)$ after the PSF anisotropy correction. portion to the fraction of unlensed cluster/foreground galaxies whose orientations are randomly distributed, thus diluting the lensing signal relative to the reference background level derived from the background population (Medezinski et al. 2007). With a pure red background sample $(B)$ as a reference, one can quantify the degree of dilution for a galaxy sample $(G)$ containing $N_{\mathrm{CL}}$ cluster galaxies and $N_{\mathrm{BG}}$ background galaxies in terms of the strengths of the averaged tangential shear signal $\left\langle g_{+}(\theta)\right\rangle$ as (Medezinski et al. 2007)

$$
\begin{aligned}
1+\delta_{d}(\theta) & \equiv \frac{N_{\mathrm{BG}}+N_{\mathrm{CL}}}{N_{\mathrm{BG}}} \\
& =\frac{\left\langle g_{+}^{(B)}(\theta)\right\rangle}{\left\langle g_{+}^{(G)}(\theta)\right\rangle} \frac{\left\langle D_{d s} / D_{s}\right\rangle_{z_{s}>z_{d}}^{(G)}}{\left\langle D_{d s} / D_{s}\right\rangle_{z_{s}>z_{d}}^{(B)}},
\end{aligned}
$$

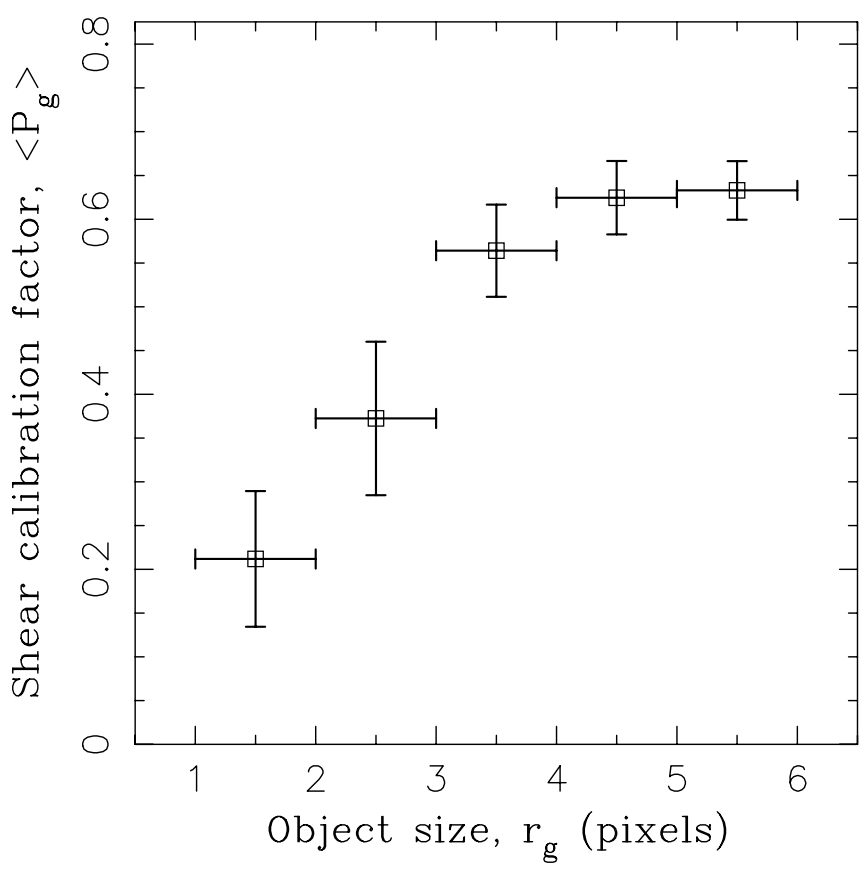

FIG. 3.- Averaged shear correction factor, $\left\langle P_{g}^{s}\right\rangle$, as a function of object size, $r_{g}$. The horizontal error bar represents the size of the bin $\left(\Delta r_{g}=1\right.$ pixel $)$, and the vertical error bar represents the rms scatter in the smoothed scalar correction factor, $\left\langle P_{g}\right\rangle$. 


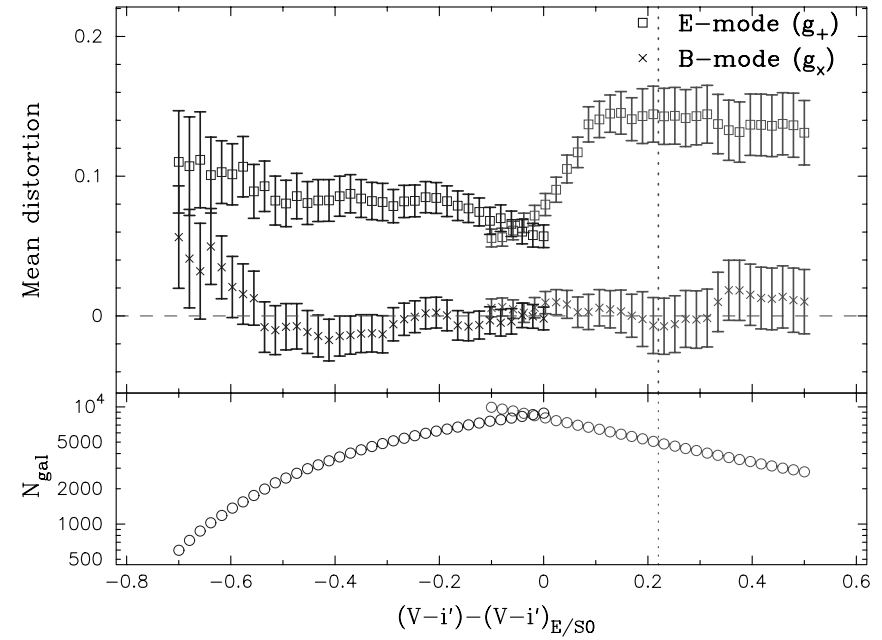

FIG. 4.-Top: Mean distortion strength averaged over a wide radial range of $1^{\prime}<\theta<18^{\prime}$, done separately for the blue and red galaxy samples. No area weighting is used here to enhance the effect of dilution in the central cluster region. Shown are the measurements of the tangential component $\left(g_{+}\right)$with open squares, and those of the $45^{\circ}$ rotated component $\left(g_{\times}\right)$. On the right (light gray symbols), the squares show that $g_{+}$drops rapidly when the bluer limit of the entire red sample is decreased below a color indicated by the vertical dashed line which lies +0.22 mag redward of the cluster sequence. This sharp decline marks the point at which the red sample encroaches on the E/S0 sequence of the cluster. For galaxies with colors bluer than the cluster sequence, cluster members are present along with background galaxies. Consequently, the mean lensing strength of the blue sample, as shown on the left (light gray symbols), is systematically lower than that of the red sample. Bottom: Respective numbers of galaxies as a function of color limit, contained in the range $1^{\prime}<\theta<18^{\prime}$ in the red (right) and the blue (left) samples. [See the electronic edition of the Journal for a color version of this figure.]

where $\left\langle D_{d s} / D_{s}\right\rangle_{z_{\mathrm{s}}>z_{d}}$ values are averaged distance ratios for respective background populations; if the two samples contain the same background population, then $\delta_{d}=\left\langle g_{+}^{(B)}\right\rangle /\left\langle g_{+}^{(G)}\right\rangle-1$. The degree of dilution thus varies depending on the radius from the cluster center, increasing toward the cluster center. Medezinski et al. (2007) found for their green galaxy sample $\left[\left(V-i^{\prime}\right)_{\mathrm{E} / \mathrm{S} 0-0.3}^{+0.1}\right.$ containing the cluster sequence galaxies in A1689 that the fraction of cluster membership, $N_{\mathrm{CL}} /\left(N_{\mathrm{BG}}+N_{\mathrm{CL}}\right)$, tends $\sim 100 \%$ within $\theta \lesssim 2^{\prime}$.

For our weak-lensing distortion analysis we define a sample of red background galaxies whose colors are redder due to large $k$-corrections than the CM relation, or red sequence, of cluster member galaxies. These red background galaxies are largely composed of early to mid-type galaxies at moderate redshifts (Medezinski et al. 2007). Cluster member galaxies are not expected to extend to these colors in any significant numbers because the intrinsically reddest class of cluster galaxies, i.e., E/S0 galaxies, are defined by the red sequence and lie blueward of the chosen sample limit, so that even large photometric errors will not carry them into our red sample. This can be demonstrated readily, as shown in Figure 4, where we plot the mean tangential shear strength $\left\langle g_{+}\right\rangle$, averaged over a wide radial range of $1^{\prime}<$ $\theta<18^{\prime}$, as a function of color limit by changing the lower color limit progressively blueward. Here we do not apply area weighting to enhance the effect of dilution in the central region. We take the lower (bluer) color limit of $+0.22 \mathrm{mag}$ where the cluster contribution is negligible, defining the red background sample by $\Delta\left(V-i^{\prime}\right) \equiv\left(V-i^{\prime}\right)-\left(V-i^{\prime}\right)_{\mathrm{E} / \mathrm{S} 0}>0.22$, as adopted by B05a. Figure 4 shows a sharp drop in the lensing signal at $\Delta\left(V-i^{\prime}\right) \lesssim$ 0.1 , when the cluster red sequence starts to contribute significantly, thereby reducing the mean lensing signal. At $\Delta(V-$ $\left.i^{\prime}\right) \gtrsim 0.1$, the mean lensing signal of the red background stays fairly constant, $\left\langle g_{+}\right\rangle \simeq 0.143$, ensuring that our weak-lensing measurements are not sensitive to this particular choice of the color limit (see $\S 5.5$ ). Similarly, on the left of Figure 4, we show the mean distortion strength for our blue galaxy sample with the magnitude limit in the interval $23<i^{\prime}<25.5$, so as to take only faint blue galaxies. The lower color limit is set to $\Delta\left(V-i^{\prime}\right)>$ -1.5 . For galaxies with colors bluer than the cluster sequence, cluster galaxies are present along with background galaxies, since the cluster population extends to bluer colors of the later type members. Consequently, the mean lensing signal of the blue sample is systematically lower than that of the red sample, unless we take the upper (redder) color limit around $\Delta\left(V-i^{\prime}\right) \sim 0.7$, where, however, the distortion measurement is quite noisy (Fig. 4). We therefore exclude blue galaxies from our weak-lensing analysis. Note that the background populations do not need to be complete in any sense but should simply be well defined and contain only background. This color-magnitude selection criteria yielded a total of $N_{g}=5728$ galaxies, or the mean surface number density of $\bar{n}_{g}=7.6$ galaxies $\operatorname{arcmin}^{-2}$. For the red background sample, we found $\overline{\sigma_{g}^{2}} \simeq 0.133$, or $\left(\overline{\sigma_{g}^{2}}\right)^{1 / 2} \approx 0.36$, which is slightly smaller than the rms dispersion for the $i^{\prime}$ selected galaxy sample.

We need to estimate the depths of our color-magnitudeselected red samples when measuring the cluster mass profile, because the lensing signal depends on the source redshifts in proportion to $D_{d s} / D_{s}$. Medezinski et al. (2007) utilized the multicolor photometry of Capak et al. (2004) based on Subaru UBVRIZ imaging covering $0.2 \mathrm{deg}^{2}$, and estimated photometric redshifts for color-magnitude-selected background galaxy samples. Using the Capak et al.'s photometric redshift distributions, Medezinski et al. (2007) estimated a mean redshift for the red background with $i^{\prime}>18$ mag to be $\bar{z}_{S} \approx 0.87$ at fainter magnitude limits of $i^{\prime}=26-27$, and also calculated weighted mean lensing depths $\left\langle D_{d s} / D_{s}\right\rangle$ for respective background populations as a function of apparent $i^{\prime}$ limiting magnitude, and found that the averaged distance ratio $\left\langle D_{d s} / D_{s}\right\rangle$ grows only slowly with increasing apparent magnitude limit: $\left\langle D_{d s} / D_{s}\right\rangle=0.693 \pm 0.02$ for the red sample with $i_{\text {cut }}^{\prime}=25.5-26.5$ (see Fig. 9 of Medezinski et al. 2007), and the corresponding redshift equivalent to this mean distance is $z_{s, D}=0.68 \pm 0.05$, where the distance-equivalent redshift $z_{s, D}$ is defined by the following equation:

$$
\left\langle\frac{D_{d s}}{D_{s}}\right\rangle_{z_{s}}=\left.\frac{D_{d s}}{D_{s}}\right|_{z_{s}=z_{s, D}} .
$$

Therefore, we can safely assume that our two different red samples for the distortion and magnification measurements (with $i_{\text {cut }}^{\prime}=26.0$ and 25.5, respectively) have nearly the same depths. We note that B05a assumed that all of the background galaxies are located at a single redshift of $z_{s}=1$, corresponding to $D_{d s} / D_{s}=0.772$, which underestimates $\Sigma_{\text {crit }}$ by $\sim 11 \%$ and hence underestimates the cluster mass accordingly. We will come back to this issue in $\S 5.5$.

\section{WEAK-LENSING MAPMAKING}

This section describes a maximum entropy method (MEM) for reconstructing the two-dimensional cluster mass distribution from combined shape distortion and magnification bias observations. As described above (see $\S 3.3$ ), only the red selected background is used for the measurement of the lens distortion and magnification, in order to minimize the effect of dilution.

\subsection{Shape Distortion Data}

For mapmaking, we pixelize the distortion data into a regular grid of $N_{\text {data }}=21 \times 17=357$ independent pixels covering a 


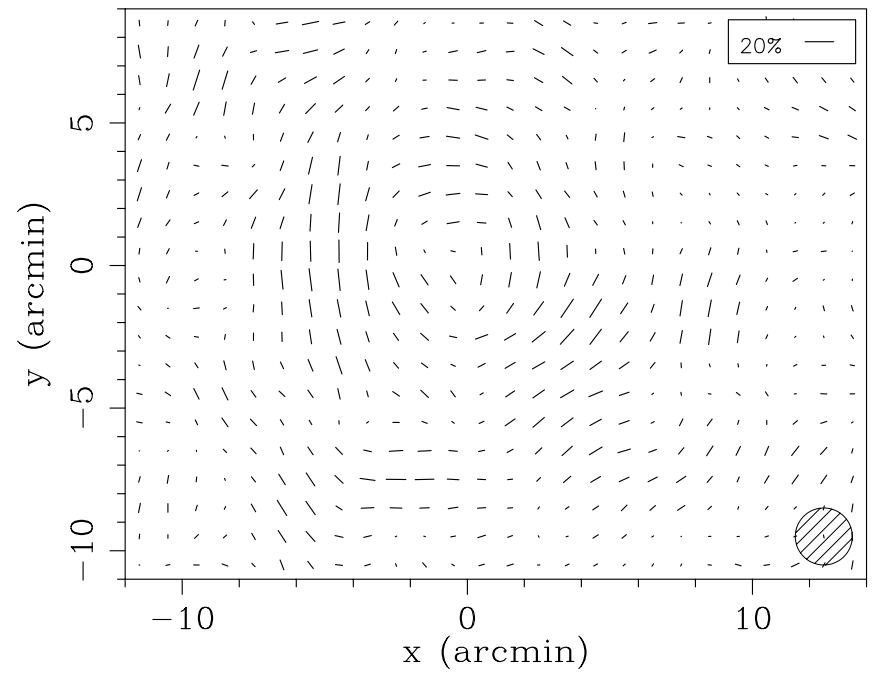

FIG. 5.-Gravitational reduced-shear field in A1689 obtained from shape distortions of the red background galaxies, smoothed with a Gaussian with $\mathrm{FWHM}=2^{\prime}$ for visualization purposes. A stick with a length of $20 \%$ ellipticity is indicated in the top right corner. The shaded circle indicates the FWHM of the Gaussian. The coordinate origin is at the optical center (see Figure 8).

field of $\approx 30^{\prime} \times 24^{\prime}$. The pixel size is set to $\Delta_{\text {pix }}=1.4^{\prime}$, and the mean galaxy counts per pixel is $\bar{n}_{g} \Delta \Omega_{\text {pix }} \sim 15$ with $\Delta \Omega_{\text {pix }}=$ $\Delta_{\text {pix }}^{2}$ being the solid angle per pixel.

We compute an estimate $\tilde{g}_{\alpha}\left(\boldsymbol{\theta}_{i}\right)$ for the reduced shear $g_{\alpha}\left(\boldsymbol{\theta}_{i}\right)$ on a regular grid of cells $\left(i=1,2, \ldots, N_{\text {pix }}\right)$ as

$$
\tilde{g}_{\alpha}\left(\boldsymbol{\theta}_{i}\right)=\sum_{k \in \operatorname{cell} i} u_{k} g_{\alpha, k} / \sum_{k \in \operatorname{cell} i} u_{k} \equiv \tilde{g}_{\alpha, i}
$$

where $g_{\alpha, k}$ is a noisy estimate of the $\alpha$ th component of the reduced shear for the $k$ th galaxy, and $u_{k}=1 / \sigma_{g, k}^{2}$ is its inversevariance weight (see $\S 3.2$ ). The error covariance matrix for the pixelized reduced shear $\tilde{g}=\tilde{g}_{1}+i \tilde{g}_{2}$ (eq. [19]) is then diagonal, and given by

$$
\left\langle\Delta \tilde{g}_{\alpha, i} \Delta \tilde{g}_{\beta, j}\right\rangle=\frac{1}{2} \sigma_{\tilde{g}, i}^{2} \delta_{\alpha \beta} \delta_{i j}
$$

where $\sigma_{\tilde{g}, j}^{2}$ is the error variance for the $j$ th pixel, defined as

$$
\sigma_{\tilde{g}}^{2}\left(\boldsymbol{\theta}_{j}\right)=\frac{1}{\sum_{k \in \operatorname{cell} j} u_{k}} .
$$

Here we have used $\left\langle\Delta g_{\alpha, k} \Delta g_{\beta, l}\right\rangle=(1 / 2) \sigma_{g, k}^{2} \delta_{\alpha \beta} \delta_{k l}$ for individual shear estimates $g_{\alpha, k}$. The per pixel rms dispersion for $\tilde{g}(\boldsymbol{\theta})$ is then reduced down to $\left(\overline{\sigma_{g}^{2}} / N\right)^{1 / 2} \sim 0.36 / \sqrt{15} \sim 0.092$.

In Figure 5 we show the reduced-shear field obtained from the red galaxy sample, where for visualization purposes the $\tilde{g}_{\alpha}(\boldsymbol{\theta})$ is resampled on to a finer grid and smoothed with a Gaussian with $\mathrm{FWHM}=2^{\prime}$. A coherent tangential shear pattern is clearly seen in Figure 5 around the cluster center.

\subsection{Magnification Bias Data}

Lensing magnification, $\mu(\boldsymbol{\theta})$, influences the observed surface density of background sources, expanding the area of sky, and enhancing the observed flux of background sources. In the subcritical regime (Broadhurst et al. 1995; Umetsu et al. 1999), the magnification $\mu(\boldsymbol{\theta})$ is given by equation (3). The count-in-cell statistic is measured from the flux-limited red galaxy sample (see $\S 3.1$ ) on the same grid as the distortion data:

$$
\tilde{N}\left(<m_{\text {cut }} ; \boldsymbol{\theta}_{i}\right)=\sum_{k \in \text { cell } i} 1 \equiv \tilde{N}_{i},
$$

with $m_{\text {cut }}$ being the magnitude cutoff corresponding to the flux limit $\left(i_{\text {cut }}^{\prime}=25.5\right)$. The normalization and slope of the unlensed number counts $N_{0}\left(<m_{\text {cut }}\right)$ for our red galaxy sample are reliably estimated as $n_{\mu, 0}=12.6 \pm 0.23 \mathrm{arcmin}^{-2}$ and $s \equiv$ $d \log N_{0}(<m) / d m=0.22 \pm 0.03$ from the outer region $\geq 10^{\prime}$ (B05a). The mean galaxy counts per pixel is thus $N_{0}=n_{\mu, 0} \Delta \Omega_{\text {pix }} \sim$ 25. The magnification bias at the $i$ th cell is then estimated as $1+\tilde{\delta}_{\mu, i}=\tilde{n}_{\mu} / n_{\mu, 0}=\tilde{N}_{i} / N_{0}$, with $\tilde{n}_{\mu, i}=\tilde{N}_{\mu, i} / \Delta \Omega_{\text {pix }}$, where the dilution effect $\delta_{d}$ is negligible for our red background sample; otherwise, $\tilde{N}_{i} / N_{0}=1+\tilde{\delta}_{\mu}+\tilde{\delta}_{d}$. The slope is less than the lensing invariant slope, $s=0.4$, and hence a net deficit of background galaxies is expected (Broadhurst et al. 1995; B05a):

$$
\delta_{\mu}(\boldsymbol{\theta}) \equiv\left\langle\tilde{\delta}_{\mu}(\boldsymbol{\theta})\right\rangle=\mu_{i}^{2.5 s-1}(\boldsymbol{\theta})-1 .
$$

In the limit of weak lensing where $\kappa,|\gamma| \ll 1, \delta_{\mu}(\boldsymbol{\theta}) \simeq$ $(5 s-2) \kappa(\boldsymbol{\theta}) \simeq-0.9 \kappa(\boldsymbol{\theta})$ with $s=0.22$.

The masking effect due to bright cluster galaxies and bright foreground objects is properly taken into account and corrected for (B05a). We conservatively account for the masking of observed sky by excluding a generous area $\pi a b$ around each masking object, where $a$ and $b$ are defined as $\nu_{\text {mask }} \equiv 3$ times the major (A_IMAGE) and minor axes (B_IMAGE) computed from SExtractor, corresponding roughly to the isophotal detection limit in our configuration (see $\S 3.1$ ). We calculate the correction factor for this masking effect as a function of radius from the cluster center, and renormalize the number density of each cell accordingly. The masking area is negligible at large radii, and increases up to $\sim 20 \%$ of the sky close to the cluster center, $\theta \lesssim 3^{\prime}$. B05a showed that the magnification bias measurements with and without the masking correction are roughly consistent with each other, and with the NFW prediction from the ACS strong-lensing observations (B05b) and the Subaru distortion profile (see Fig. 2 of B05a), even though all the measurements have different systematics. Note that if we use the masking factor $\nu_{\text {mask }}$ of 2 or 4 , instead of 3 , the results shown below remain almost unchanged (see $\S 5.5$ for more details).

Figure 6 shows the resulting magnification-bias distribution derived from the red galaxy sample based on the SExtractor photometry $(\S 3.1)$. A clear depletion of the red galaxy counts is visible in the central, high-density region of the cluster. On the other hand, it has been argued that estimates of the lensing magnification based on number counts suffer from noise arising from the intrinsic clustering of the source galaxies (e.g., Zhang \& Pen 2005). Indeed, some variance is apparent in the spatial distribution of red galaxies. In particular, a local enhancement of red background galaxies can be seen in Figure 6 around $\boldsymbol{\theta} \sim\left(0^{\prime}, 5^{\prime}\right)$, having an overdensity of $\tilde{n}_{\mu} / n_{0} \sim 1.5$. This may explain the discrepant point at $\theta \sim 5^{\prime}$ in the magnification bias profile of B05a. We will come back to this issue later in $\S 4.5$.

\subsection{Maximum Entropy Mass Reconstruction Method}

The relation between distortion and convergence is nonlocal, and the convergence $\kappa$ derived from distortion data alone suffers from a mass-sheet degeneracy (see $\S 2$ ). However, by combining the distortion and magnification measurements the convergence can be obtained unambiguously with the correct mass normalization. 


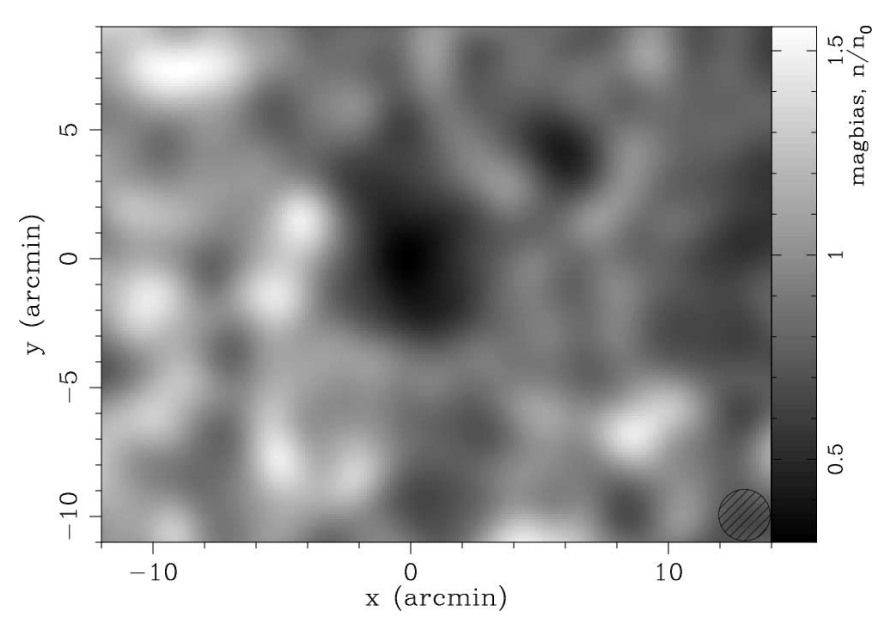

FIG. 6.-Distribution of the lensing magnification bias $n / n_{0}$ measured from a red-galaxy sample in the background of A1689, smoothed with a Gaussian with $\mathrm{FWHM}=2^{\prime}$ for visualization purposes. The shaded circle indicates the FWHM of the Gaussian.

Here we combine pixelized distortion and depletion data of red background galaxies in a maximum likelihood sense, and reconstruct the two-dimensional distribution of the lensing convergence field, $\kappa(\boldsymbol{\theta})$. Several authors have proposed maximum likelihood and maximum entropy methods for reconstructing the projected mass distribution from joint weak-lensing observations of the shape distortion and magnification bias effects (Bartelmann 1996; Seitz et al. 1998; Bridle et al. 1998; Umetsu et al. 2007). In the present study, we utilize a maximum entropy method extended to account for positive/negative distributions of the underlying field starting with the method proposed in the context of interferometric observations of the cosmic microwave background (CMB) radiation by Maisinger et al. (1997) and Hobson \& Lasenby (1998).

Bayes' theorem states that given a hypothesis $H$ and some data $D$ the posterior probability $\operatorname{Pr}(H \mid D)$ is the product of the likelihood $\operatorname{Pr}(D \mid H)$ and the prior probability $\operatorname{Pr}(H)$, with a proper normalization by the evidence $\operatorname{Pr}(D)$ (Maisinger et al. 1997):

$$
\operatorname{Pr}(H \mid D)=\frac{\operatorname{Pr}(H) \operatorname{Pr}(D \mid H)}{\operatorname{Pr}(D)}
$$

In the context of mapmaking, the hypothesis $H$ is taken as the pixelized image $\boldsymbol{p}=\left\{p_{i}\right\}\left(i=1,2, \ldots, N_{\text {pix }}\right)$. Since the evidence in Bayes' theorem is a normalization constant for a given data set $D$, we maximize $\operatorname{Pr}(\boldsymbol{p} \mid D) \propto \operatorname{Pr}(\boldsymbol{p}) \operatorname{Pr}(D \mid \boldsymbol{p})$ with respect to $\boldsymbol{p}$. We assume that the errors on the data follow a Gaussian distribution, so that the likelihood is given as $\operatorname{Pr}(D \mid \boldsymbol{p}) \propto$ $\exp \left[-\chi^{2}(\boldsymbol{p}) / 2\right]$, with $\chi^{2}$ being the standard misfit statistic. Then, for a given form of the entropy function $S(\boldsymbol{p}, \boldsymbol{m})$, the entropic prior is written as

$$
\operatorname{Pr}(\boldsymbol{p}) \propto \exp [\alpha S(\boldsymbol{p}, \boldsymbol{m})]
$$

where $\boldsymbol{m}=\left\{m_{i}\right\}\left(i=1,2, \ldots, N_{\text {pix }}\right)$ is a set of model parameters for the pixelized image, $\boldsymbol{p}$. We follow the prescription given in Maisinger et al. (1997) and Hobson \& Lasenby (1998), and define the cross-entropy function for $\boldsymbol{p}$ as

$$
S(\boldsymbol{p}, \boldsymbol{m})=\sum_{i=1}^{N_{\mathrm{pix}}}\left[\psi_{i}-2 m_{i}-p_{i} \ln \left(\frac{\psi_{i}+p_{i}}{2 m_{i}}\right)\right]
$$

where $\psi_{i} \equiv\left(p_{i}^{2}+4 m_{i}^{2}\right)^{1 / 2}$. We take $p_{i}=\kappa_{i} \equiv \kappa\left(\boldsymbol{\theta}_{i}\right)$ as the image to be reconstructed, and express a set of discretized $\kappa$-values as $\boldsymbol{p}=\left\{\kappa_{i}\right\}\left(i=1,2, \ldots, N_{\text {pix }}\right)$. In general, an entropy regularization helps to reduce the sensitivity of the least $\chi^{2}$ solutions to small-scale noise in data, by imposing smoothness constraints on the solutions. This MEM prior ensures that $\kappa(\boldsymbol{\theta}) \rightarrow 0$ in the noise-dominated regime, or low-density regions in the outskirts of the cluster. Note that unlike conventional MEM functions, this MEM prior is free from the "positive bias" in the reconstructed image (=signal + residual noise), and this bias is more significant in the low signal-to-noise ratio, or low-density regions $\left(r \rightarrow r_{\text {vir }}\right)$ where we are interested in measuring $\kappa$.

We take into account the nonlinear, but subcritical, regime of the lensing properties, $\kappa$ and $\gamma_{\alpha}$, for a MEM mass reconstruction (see Bridle et al. 1998). We use equations (7) and (9) to relate the gravitational shear and the convergence fields, $\gamma$ and $\kappa$. The log posterior probability function, $F(\boldsymbol{p})=-\ln \operatorname{Pr}(\boldsymbol{p} \mid D)$, is then expressed as a linear sum of the shear/magnification data log likelihoods (Schneider et al. 2000) and the entropy term (Maisinger et al. 1997):

$$
\begin{aligned}
F(\boldsymbol{p}) & =\frac{1}{2} \chi^{2}(\boldsymbol{p})-\alpha S(\boldsymbol{p}, \boldsymbol{m}), \\
\chi^{2}(\boldsymbol{p}) & =\chi_{g}^{2}(\boldsymbol{p})+\chi_{\mu}^{2}(\boldsymbol{p}) \\
\chi_{g}^{2} & \equiv \sum_{i=1}^{N_{\mathrm{pix}}} \sum_{\alpha=1}^{2} \frac{\left[\tilde{g}_{\alpha, i}-g_{\alpha, i}(\boldsymbol{p})\right]^{2}}{\sigma_{\tilde{g}_{\alpha}, i}^{2}}, \\
\chi_{\mu}^{2} & \equiv \sum_{i=1}^{N_{\mathrm{pix}}} \frac{\left[\tilde{N}_{i}-N_{i}(\boldsymbol{p})\right]^{2}}{\tilde{N}_{i}},
\end{aligned}
$$

where $\alpha(>0)$ is the dimensionless regularization constant, and $\sigma_{\tilde{g}_{\alpha, i}}$ is the per component rms error for the pixelized distortion measurement $(\alpha=1,2), \sigma_{\tilde{g}_{1, i}}=\sigma_{\tilde{g}_{2, i}}=\sigma_{\tilde{g}, i} / \sqrt{2} ; g_{\alpha, i}(\boldsymbol{p})$ (eq. [10]) and $N_{i}(\boldsymbol{p})=\mu_{i}(\boldsymbol{p})^{2.5 s-1} N_{0}$ are the theoretical expectations for $\tilde{g}_{\alpha, i}$ and $\tilde{N}_{i}$, respectively, where $\Delta \Omega_{\text {pix }, i}$ is the effective observed area of the $i$ th pixel excluding the masking area by the cluster members. In defining $\chi_{\mu}^{2}$, we have used the Gaussian approximation for the Poisson distribution of count-in-cell statistics $\left(N_{0} \sim 25\right)$, as done in the one-dimensional analysis by B05a. We also note that the dispersion for $\tilde{g}$ is modified as $\sigma[\tilde{g}(\boldsymbol{\theta})] \approx \sigma_{\tilde{g}}(1-$ $\left.|g(\boldsymbol{p})|^{2}\right)$ in the subcritical, nonlinear regime (Schneider et al. 2000); however, we neglect this nonlinear correction for the dispersion $\sigma_{\tilde{g}}$ to simplify various calculations, as in B05a. We found these are indeed good approximations for our combined distortion and depletion data sets, and the results presented here are little changed when the full likelihood function is used.

The maximum likelihood solution, $\hat{\boldsymbol{p}}$, is obtained by minimizing the function $F(\boldsymbol{p})$ with respect to $\boldsymbol{p}$ for given $\alpha$ and $\boldsymbol{m}$. To do this, we compute numerically the derivatives $\partial F(\boldsymbol{p}) / \partial p_{i}$ using a conjugate-gradient algorithm (Press et al. 1992). We determine by iteration the Bayesian value of $\alpha=\hat{\alpha}(\hat{\boldsymbol{p}}, \boldsymbol{m})$ by the following equation (Bridle et al. 1998):

$$
-2 \hat{\alpha} S(\hat{\boldsymbol{p}}, \boldsymbol{m})=N_{\mathrm{pix}}-\hat{\alpha} \operatorname{Tr}\left(\boldsymbol{M}^{-1}\right) \equiv N_{\mathrm{good}}
$$

where $\boldsymbol{M}$ is a $N_{\text {pix }} \times N_{\text {pix }}$ matrix defined by

$$
\boldsymbol{M}=\boldsymbol{G}^{-1 / 2} \boldsymbol{H} \boldsymbol{G}^{-1 / 2},
$$


with

$$
\begin{aligned}
& H_{i j}(\hat{\boldsymbol{p}})=\left.\frac{\partial^{2} F(\boldsymbol{p})}{\partial p_{i} \partial p_{j}}\right|_{\boldsymbol{p}=\hat{\boldsymbol{p}}}, \\
& G_{i j}(\hat{\boldsymbol{p}})=\left.\frac{\partial^{2} S(\boldsymbol{p})}{\partial p_{i} \partial p_{j}}\right|_{\boldsymbol{p}=\hat{\boldsymbol{p}}},
\end{aligned}
$$

evaluated at $\boldsymbol{p}=\hat{\boldsymbol{p}} ; N_{\mathrm{good}}$ is a measure of the effective number of parameters (Suyu et al. 2006). With the optimal $\alpha$, we thus expect that the final value of the misfit statistic $\chi^{2}$ is close to the classical number of degrees of freedom (hereafter NDF; see Suyu et al. 2006), NDF $\equiv N_{\text {data }}-N_{\text {good }}$ (classic MEM). We take $m_{i}=$ const. $\equiv m$, where $m$ can be regarded as a characteristic amplitude of the image (Maisinger et al. 1997). We find that the maximum likelihood solution $\hat{\boldsymbol{p}}$ for the Bayesian $\hat{\alpha}$ is insensitive to the choice of $m$ (see $\S 5.5$ ). In the following we set $m$ to be 0.5 . In order to be able to quantify the errors on the mass reconstruction we evaluate the Hessian matrix $\boldsymbol{H}$ of the function $F(\boldsymbol{p})$ at $\boldsymbol{p}=\hat{\boldsymbol{p}}$, from which the covariance matrix of the reconstructed $\kappa$ map is given by

$$
C_{i j} \equiv\left\langle\delta \kappa_{i} \delta \kappa_{j}\right\rangle=(H)_{i j}^{-1}(\hat{\boldsymbol{p}}) .
$$

\subsection{Strong-Lensing Constraints}

The mapmaking method described in $\S 4.3$ is only applicable to subcritical regions lying outside of the critical curves (see Bartelmann \& Schneider 2001). Therefore, our Subaru distortion and depletion data sets cannot be used to constrain the cluster mass distribution in the strong-lensing region within the tangential critical curve (see B05b). Instead, we can pause quadratic constraints on such pixels to the total log likelihood function based on strong-lensing models as

$$
F(\boldsymbol{p}) \rightarrow F(\boldsymbol{p})+\frac{\beta}{2} \sum_{i=1}^{N_{c}}\left(\kappa-\kappa_{c}\right)_{i}^{2},
$$

where $\kappa_{c . i}$ is the constraint on $\kappa_{i}$ by strong lensing, $N_{c}$ is the number of constraints, and $\beta$ is a large number without causing this external term to become singular to working precision.

In the present pixelization scheme, there is one such pixel $\left(N_{c}=1\right)$ containing the strong-lensing region for which the Einstein radius of $\theta_{\mathrm{E}} \simeq 43^{\prime \prime}$ corresponds to a mean redshift of $\bar{z}_{s}=0.87$ for our red background population (see $\S 3.3$ ). To constrain the central $\kappa$ pixel, we utilized a mass model of A1689, which is well constrained by ACS strong-lensing observations restricted to the central region $\lesssim 2^{\prime}(\mathrm{B} 05 \mathrm{~b})$. The central ACSderived mass profile of $\mathrm{B} 05 \mathrm{~b}$ is best described by an NFW model with a virial mass $M_{\mathrm{vir}}=2.6 \times 10^{15} M_{\odot} h^{-1}$ and a concentration $c_{\mathrm{vir}}=8.2$ having a scale radius of $r_{s} \equiv r_{\mathrm{vir}} / c_{\mathrm{vir}}=310 \mathrm{kpc} h^{-1}$ (B05b). With this NFW model, we find $\kappa_{c} \approx 0.781 \pm 0.1$ at the central pixel of $\boldsymbol{\theta} \sim\left(0^{\prime}, 0^{\prime}\right)$ for a reference source redshift of $z_{s}=1$, corresponding to $\kappa_{c} \approx 0.700$ for our red background sample ( $(3.3)$. Hence, assuming $z_{s}=1$ for the source redshift will overpredict the central density by a factor of $0.772 / 0.693 \simeq$ 1.113 , which will affect slightly the overall amplitude of the reconstructed $\kappa$ map (see $\S 5.5$ ).

\subsection{Downweighting Intrinsic Clustering Contributions and Noisy Measurements}

In contrast to the shearing effect, the magnification bias is a local, direct measure of the lensing convergence, free from the mass-sheet degeneracy. However, a practical difficulty of the magnification bias measurement is the intrinsic clustering contribution which locally can be larger than the lensing-induced signal in a given pixel. In order to obtain a clean measure of the lensing signal, such intrinsic clustering needs to be eliminated. Broadhurst et al. (1995) proposed an active declustering method based on the facts that the magnification bias depends strongly on the shape of the luminosity function, whereas intrinsic clustering depends weakly on the intrinsic luminosity function, and that they have different redshift dependence (see also Zhang \& Pen 2005). This method however requires the addition of redshift information for individual background galaxies. Furthermore, the shape information of luminosity functions for respective background populations must be provided in order to convert a density depletion or enhancement into the lens magnification, $\mu$.

Alternatively, one may employ prior information from, for example, the surface luminosity density of cluster member galaxies, to predict the projected mass distribution, which is then used to downweight intrinsic clustering contributions. In the present study, we have adopted an objective rejection scheme based on the gravitational shear predictions, summarized in the following steps: (1) Using shape distortion data alone we derive an entropy-regularized maximum likelihood solution $\boldsymbol{p}$ for the mass distribution. (2) Then, the shear-based $\kappa$ map is used to predict the magnification bias on the same grid of pixels. (3) Finally, we make a pixel-to-pixel comparison between the observed and predicted galaxy counts, and reject those magnification measurements $\tilde{N}_{i}$ which are in conflict with the shear-based predictions $N_{i}(\boldsymbol{p})$ :

$$
\left|\tilde{N}_{i}-N_{i}(\boldsymbol{p})\right|>\nu_{\text {clust }} \sqrt{N_{0}},
$$

where $N_{0} \sim 25$ is the unlensed, mean number counts of red galaxies, and $\nu_{\text {clust }}$ is a rejection threshold in units of $\sigma$. We set a rejection threshold at $4 \sigma$, i.e., $\nu_{\text {clust }}=4$. Changing this threshold will affect the details of the reconstruction especially in the outer regions $\left(\theta \gtrsim 7^{\prime}\right)$. However, we confirmed that changing the threshold $\nu_{\text {clust }}$ by \pm 1 leaves our results unchanged within our statistical uncertainties (see $\S 5.5$ ), and that the resulting mass map is fairly consistent with the surface luminosity and density distributions of cluster member galaxies, as described in detail below.

In addition to the above, in order to exclude unreliable measurements, we have assigned zero weight to those pixels which satisfy either of the following rejection criteria:

1. Measurement pixel with no usable galaxy.

2. Measurement pixel in the strong-lensing region (§ 4.4).

3. Magnification measurement near boundary regions with low completeness at fainter magnitudes.

The last rejection criterion is required since the slope of the unlensed number counts, $s=d \log N_{0}(<m) / d m$, could depend on the selection completeness; such an apparent variation of the slope $s$ could give rise to additional errors in the mass reconstruction.

\subsection{Mapmaking of A1689}

We have applied our MEM method to the joint measurements of the shape distortion and magnification bias effects of red background galaxies in order to reconstruct the two-dimensional mass distribution of A1689. We utilized the FFTW implementation of fast Fourier transforms (FFTs) to convert between the lensing convergence and the gravitational shear fields using equations (7) and (9). The FFT however implies a periodicity in both horizontal and vertical directions, producing aliasing effects at the borders of the computational domain. In order to avoid such 
TABLE 1

Parameters Used in the 2D MEM Reconstruction

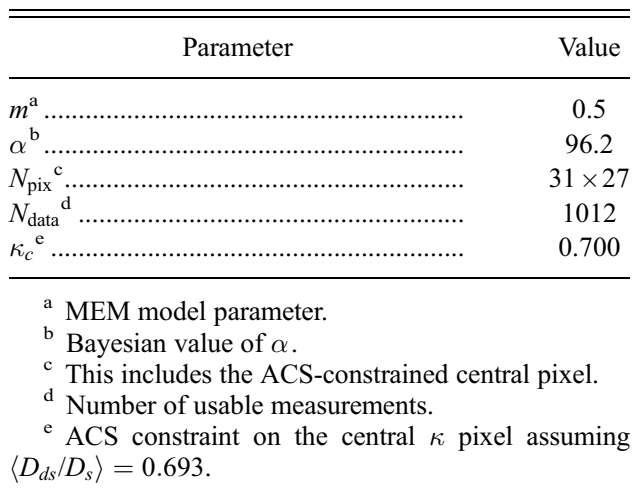

aliasing effects, we used large arrays of $31 \times 27$ pixels with $N_{\text {pix }}=31 \times 27=837$, covering a field of $43.4^{\prime} \times 37.8^{\prime}$. On the other hand, the distortion and depletion measurements are limited to the central $30^{\prime} \times 24^{\prime}$ region $(21 \times 17=357$ pixels $)$; the wide-field Subaru data thus allow us to probe the projected mass distribution on scales ranging from $\theta \sim 1^{\prime}$ up to $\sim 20^{\prime}$. To minimize spurious effects from the periodic boundary condition, pixelized maps are further zero padded by a factor of 2 in each dimension (Seljak 1998; Sato et al. 2003).

Table 1 summarizes the parameters used in our 2D MEM reconstruction. For the spin-2 distortion measurements, we have in total $N_{\text {data }, g}=2 \times 355=710$ usable (real) observations. For the magnification measurements, we have $N_{\text {data }, \mu}=302$ usable observations. The total number of constraints is thus $N_{\text {data }}=$ $N_{\text {data }, g}+N_{\text {data }, \mu}=1012$, yielding $N_{\text {data }}-N_{\text {pix }}=175$ degrees of freedom (dof). The Bayesian value of the regularization parameter $\hat{\alpha}$ that satisfies equation (31) was obtained as $\hat{\alpha}=96.6$, when $-2 \hat{\alpha} S \approx N_{\text {pix }}-\hat{\alpha} \operatorname{Tr}\left(\boldsymbol{M}^{-1}\right) \approx 213.1\left(=N_{\text {good }}\right)$. The minimum function value of $F$ was found to be $F_{\min }=F(\hat{\boldsymbol{p}})=$ $(1 / 2) \chi^{2}(\hat{\boldsymbol{p}})-\hat{\alpha} S(\hat{\boldsymbol{p}})=651.2$, and $\chi^{2}(\hat{\boldsymbol{p}})=1089.3$, corresponding to $\mathrm{NDF}=N_{\text {data }}-N_{\text {good }}=798.9$, i.e., $\chi^{2}(\hat{\boldsymbol{p}}) / \mathrm{NDF}=1.36$. Hence, the resulting $\chi^{2}$ is somewhat large, and is rather close to historic MEM, i.e., $\chi^{2}(\hat{\boldsymbol{p}}) \approx N_{\text {data. }}$. However, we note that our MEM mass reconstructions without the magnification data yield $\chi^{2}(\hat{\boldsymbol{p}}) / \mathrm{NDF} \approx 1$ (see Table 2 ) as expected for classic MEM. Therefore, slightly large values of this misfit statistic using the magnification data could be attributed to the fact that the intrinsic clustering noise in the magnification measurements is not included in the likelihood calculation, underestimating the errors for the magnification measurements.
In the left panel of Figure 7 we show the resulting $\kappa$ map on a grid of $31 \times 27$ pixels reconstructed from the combined distortion and depletion data with the ACS constraint on the mean value of $\kappa$ for the central pixel. The reconstructed spin- 2 shear field $[\gamma(\hat{\boldsymbol{p}})]$ is overlaid upon the $\kappa$ map. The right panel in Figure 7 shows the two-dimensional distribution of the rms reconstruction error for $\kappa_{i}$, estimated from the diagonal part of the pixel-pixel covariance matrix of errors: $\sigma\left(\kappa_{i}\right)=C_{i i}^{1 / 2}(\hat{p})$. Figure 8 presents the contours of the reconstructed $\kappa$ map superposed on the $V+i^{\prime}$ pseudocolor image covering a field of $30^{\prime} \times 25^{\prime}$ around the cluster. Here, for visualization purposes, the $\kappa$ map is resampled on to a finer grid and convolved with a Gaussian of FWHM $=1.4^{\prime}$, corresponding to a physical resolution of FWHM $\sim 180 \mathrm{kpc} h^{-1}$ at the cluster redshift. The projected mass distribution of the cluster is smoothly varying and symmetric, with no significant substructure at $r \gtrsim 100 \mathrm{kpc}^{-1}$. Figure 9 compares the reconstructed lensing fields and the member galaxy distributions in A1689, Gaussian smoothed to a resolution of FWHM $=2^{\prime}$. The top panels show the reconstructed $\kappa$ and the magnification bias $\delta_{\mu}=\mu^{2.5 s-1}-1$ fields in the left and right panels, respectively. The bottom left and right panels display the field-corrected $i^{\prime}$-band luminosity and number density maps, respectively, of bright red-sequence galaxies with $i^{\prime}<23 \mathrm{mag}$. For each panel the color scale is linear, and ranges from $0 \%-100 \%$ of the peak value. It is clear from Figure 9 that mass and light in A1689 are similarly distributed with a fairly round shape, and well centered on the main $\mathrm{cD}$ galaxy.

\subsection{Mass Maps from Different Data Sets and Boundary Conditions}

Any mass reconstruction technique based on the shear information involves a nonlocal process, meaning that one has to assume certain boundary conditions to convert the gravitational shear field, $\gamma(\boldsymbol{\theta})$, into the lensing convergence field, $\kappa(\boldsymbol{\theta})$ (Bartelmann $\&$ Schneider 2001; Umetsu et al. 1999). If the data field is sufficiently large so as to ensure that projected mass fluctuations over the field average out, or that the mean convergence over the field is zero, then one may simply use equation (7) to invert the observed shear field into the convergence field (Kaiser \& Squires 1993). Or, if magnification data are available, then a combination of complementary shearing and magnification information can be used to break the degeneracy between the observables and the underlying gravitational potential field (Bartelmann et al. 1996; Seitz et al. 1998). Besides, strong-lensing observations, if available, will place additional, tight constraints on the central mass distribution of the cluster where weak lensing alone cannot

TABLE 2

2D MEM Reconstructions with Different Data Sets and Boundary Conditions

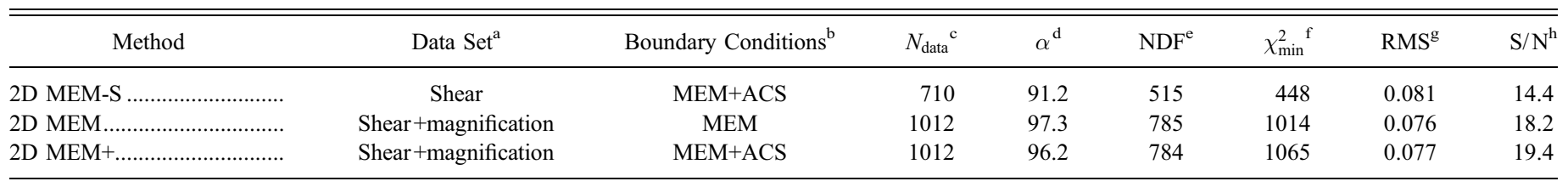

NoTEs.-The following three sets of combinations of data sets and boundary conditions are considered: (1) 2D MEM+ method using shear and magnification data with the ACS constraint on the central pixel, (2) 2D MEM method using shear and magnification data without the central ACS constraint, and (3) 2D MEM-S method using shear data with the central ACS constraint.

${ }^{a}$ Data set used for weak-lensing analysis.

${ }^{\mathrm{b}}$ With or without the ACS constraint on the central pixel in the strong-lensing region.

c Number of usable measurements.

d Bayesian value of $\alpha(m=0.5)$.

e Classical number of degrees of freedom, NDF $\equiv N_{\text {data }}-N_{\text {good }}$ (see eq. [31]).

${ }^{\mathrm{f}}$ Minimum functional value of $\chi^{2}$.

g Average rms error of $\kappa$ in the observed region $\left(30^{\prime} \times 24^{\prime}\right)$.

h Detection significance of the convergence signal defined by eq. (38). 

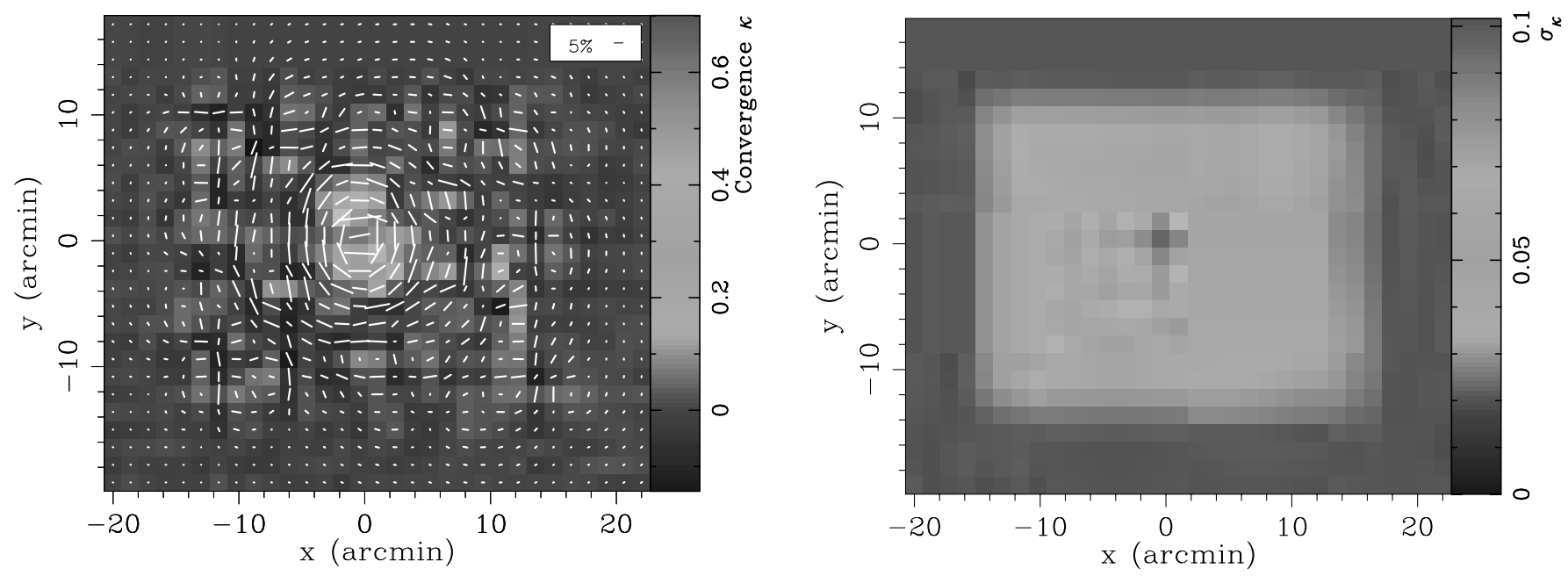

FIG. 7.-Left: Projected mass distribution $\kappa$ of A1689 on a grid of $31 \times 27$ pixels reconstructed from an entropy-regularized, maximum likelihood combination of Subaru shape distortion and number count depletion data of red background galaxies. ACS strong-lensing constraints are used to determine the convergence value at the central pixel that falls in the strong-lensing region. The pixel width is $1.4^{\prime}$. The observing region is limited to the central $30^{\prime} \times 24^{\prime}$, covering a projected area of $3.9 \times$ $3.1 \mathrm{Mpc}^{2} h^{-2}$, while the field size for the reconstruction is $43.4^{\prime} \times 37.8^{\prime}$. Overlaid on the image is the reconstructed spin-2 gravitational shear field. A stick with a length of $5 \%$ shear is indicated in the top right corner. The north is to the top, and the east is to the left. Right: Distribution of the reconstruction errors for the convergence $\kappa$ calculated using the maximum entropy method. The uncertainties are given by the square root of the diagonal part of the covariance matrix. [See the electronic edition of the Journal for a color version of this figure.]

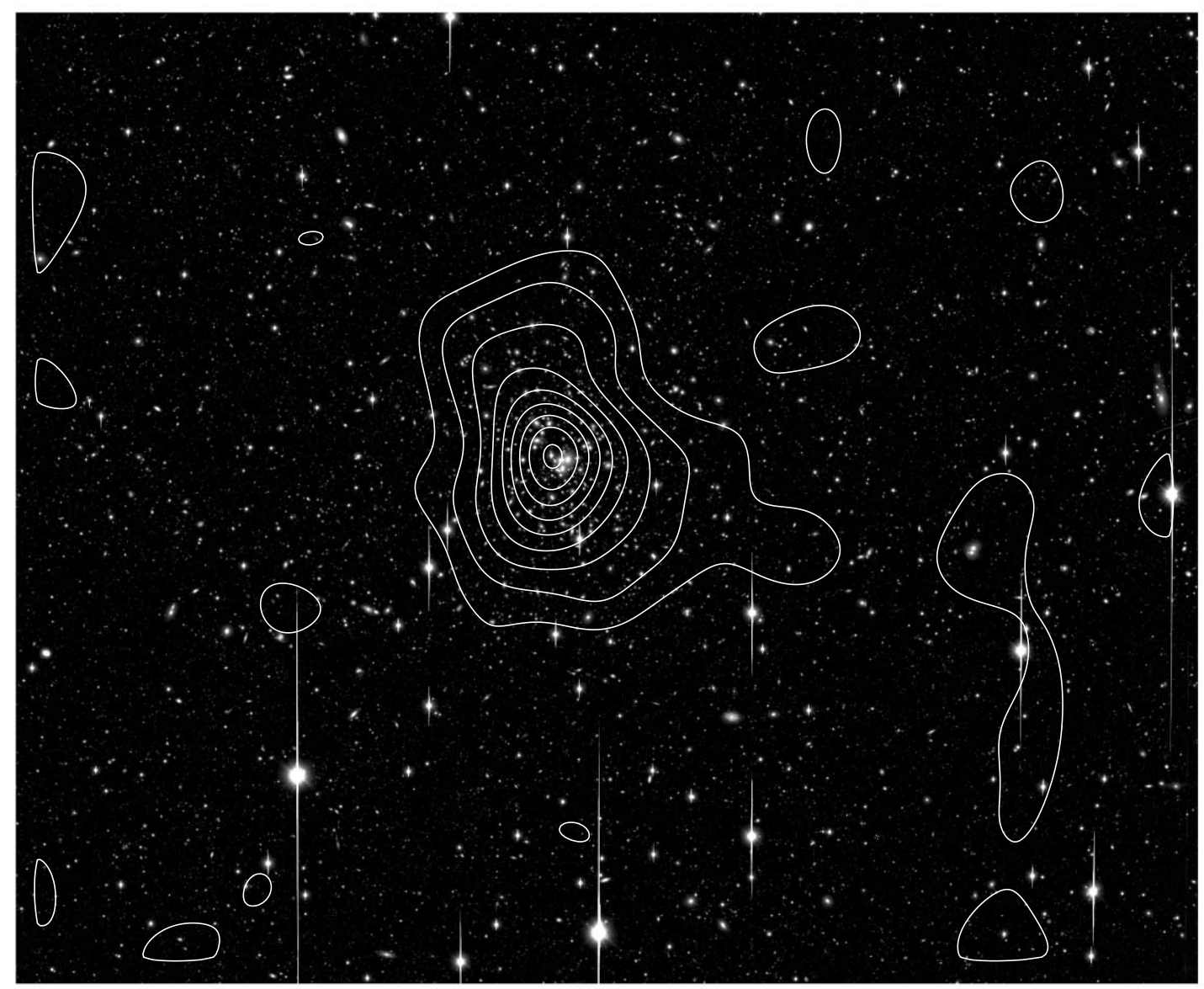

FIG. 8. - Contours of the dimensionless surface mass density $\kappa$ smoothed with a Gaussian of FWHM $=1.4^{\prime}$, superposed on the $V+i^{\prime}$ pseudocolor image of A1689. The image size is $\approx 30^{\prime} \times 25^{\prime}$, covering a projected area of $3.9 \times 3.2 \mathrm{Mpc}^{2} h^{-2}$ at $z=0.183$. The lowest contour and the contour interval are 0.05 . North is to the top and east is to the left. [See the electronic edition of the Journal for a color version of this figure.] 

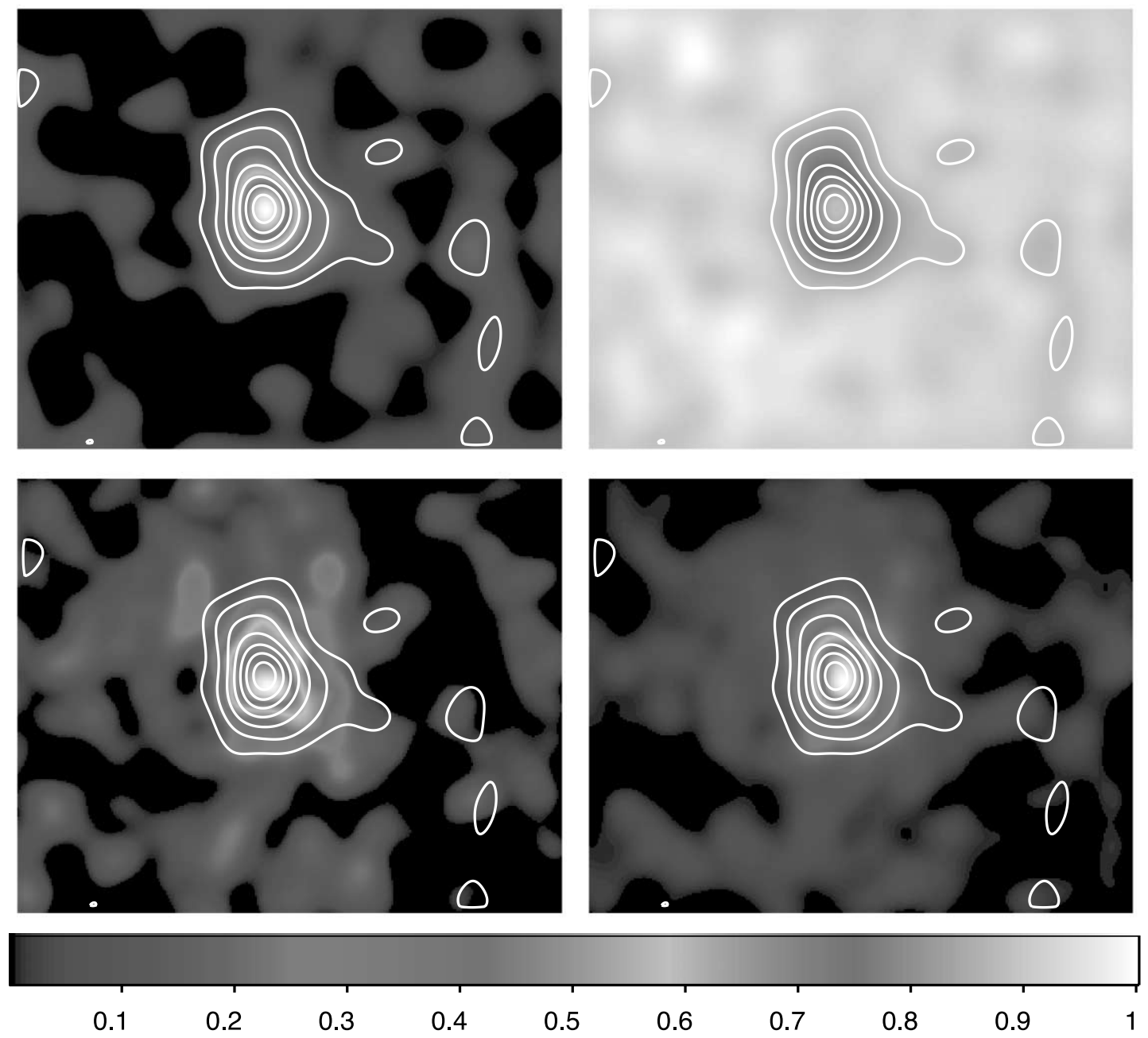

FIG. 9.-Comparison of the reconstructed lensing fields and the cluster galaxy distributions in A1689. Top: Reconstructed convergence $\kappa$ (left) and magnification bias $\mu^{2.5 s-1}$ (right) fields. Bottom: Observed $i^{\prime}$-band surface luminosity (left) and number (right) density distributions of $\left(V-i^{\prime}\right)$-selected member galaxies with $i^{\prime}<$ $23 \mathrm{ABmag}$. All images are smoothed with a Gaussian of FWHM $=2^{\prime}$. The field size is $30^{\prime} \times 24^{\prime}$. North is to the top, east to the left. The contours show the Gaussian smoothed convergence distribution shown in the top left panel. The lowest contour level and the contour interval are 0.05 . For each panel the color scale is linear, and ranges from $0 \%$ to $100 \%$ of the peak value. [See the electronic edition of the Journal for a color version of this figure.]

constrain (e.g., mass reconstruction of A370 in Umetsu et al. 1999).

Here we consider three sets of combinations of data sets and boundary conditions as summarized in Table 2: (1) $2 \mathrm{D} \mathrm{MEM+}$ method using shear and magnification data with the central ACS constraint, (2) 2D MEM method using shear and magnification data without the ACS constraint, and (3) 2D MEM-S method using shear data with the central ACS constraint. For each MEM reconstruction, we derive an entropy-regularized maximum likelihood solution for $\kappa$ with the Bayesian value of $\alpha$. The resulting Bayesian value of $\alpha$ and the minimized functional values of $F$ and $\chi^{2}$ are also listed in Table 2 .

In order to quantify the significance of the reconstruction, we define an estimator for the detection signal-to-noise ratio $(\mathrm{S} / \mathrm{N})$ by the following equation:

$$
(\mathrm{S} / \mathrm{N})^{2} \equiv \sum_{\kappa_{i}>0} \sum_{\kappa_{j}>0} \kappa_{i} \kappa_{j} C_{i j}^{-1},
$$

where the indices $i$ and $j$ run over all pixels except those with negative values of $\kappa$ and those in the strong-lensing region (see Table 2). The reconstructions based on both the distortion and depletion data yield a similar $\mathrm{S} / \mathrm{N}$ of $\sim 18-19$, whereas the reconstruction from the distortion data alone gives a slightly lower $\mathrm{S} / \mathrm{N}$ of $\sim 15$ (see Table 2 ). For comparison we quote the detection significance from the 1D Subaru analysis by B05a based on the same red background catalogs for the weak-lensing distortion and depletion analyses: B05a measured the lens distortion and depletion profiles over a radial range of $1^{\prime} \lesssim \theta \lesssim 18^{\prime}$. We find $\mathrm{S} / \mathrm{N} \simeq 14.2$ and 9.2 for the measurements of the distortion and depletion profiles, respectively. Since covariance between the distortion and magnification measurements can be neglected, the total $\mathrm{S} / \mathrm{N}$ is simply obtained as $\left(14.2^{2}+9.2^{2}\right)^{1 / 2} \sim 17$. These numbers are quite comparable to those as measured from the reconstructed $\kappa$ field and its covariance matrix, indicating that the lensing information contained in the red catalogs is properly propagated into the $\kappa$-basis. ${ }^{7}$ We find it important to use the Bayesian value for $\alpha$ in order to have an optimal smoothness for the mass reconstruction, avoiding oversmoothing. A more

7 This is not trivial since the noise level in a MEM-reconstructed map is affected by the smoothness constraint specified by $\alpha$. Besides, MEM is nonlinear, so that the resulting reconstruction errors depend on the signal as well as noise properties. 
TABLE 3

Summary of the Methods for Mass Profile Reconstructions

\begin{tabular}{|c|c|c|c|c|}
\hline Method $^{\mathrm{a}}$ & Data Set ${ }^{\mathrm{b}}$ & Boundary Conditions ${ }^{\mathrm{c}}$ & $\left(\theta_{\min }, \theta_{\max }\right)^{\mathrm{d}}$ & $N_{\text {bin }}{ }^{\mathrm{e}}$ \\
\hline 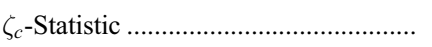 & Tangential shear & $\bar{\kappa}_{b}=4 \times 10^{-3 \mathrm{f}}$ & $\left(1^{\prime}, 16^{\prime}\right)$ & 9 \\
\hline 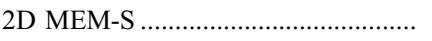 & Shear & $\mathrm{MEM}+\mathrm{ACS}$ & $\left(1^{\prime}, 18^{\prime}\right)$ & 10 \\
\hline (1) & Shear + magbias & MEM & $\left(1^{\prime}, 18^{\prime}\right)$ & 10 \\
\hline 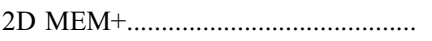 & Shear + magbias & $\mathrm{MEM}+\mathrm{ACS}$ & $\left(1^{\prime}, 18^{\prime}\right)$ & 10 \\
\hline Subaru $1 \mathrm{D}^{\mathrm{g}} \ldots$ & Tangential shear + magbias & ACS & $\left(1^{\prime}, 18^{\prime}\right)$ & 10 \\
\hline
\end{tabular}

${ }^{\text {a }}$ Weak-lensing mass reconstruction method. All methods apply to the nonlinear but subcritical regime.

${ }^{\mathrm{b}}$ Data set used for weak-lensing analysis.

${ }^{c}$ With or without the ACS constraint on the central pixel in the strong-lensing region.

${ }^{\mathrm{d}}$ Lower and upper radial limits.

e Number of radial bins in the range of $\left(\theta_{\min }, \theta_{\max }\right)$.

${ }^{\mathrm{f}}$ This employs an outer boundary condition on the mean convergence $\bar{\kappa}_{b}$ within $16^{\prime}<\theta<19^{\prime}$. The mean background level $\bar{\kappa}_{b}$ is calculated to be $\bar{\kappa}_{b}=4 \times 10^{-3}$ using the ACS + Subaru-1D best-fit NFW model by B05a.

${ }^{g}$ One-dimensional maximum likelihood analysis by B05a based on the joint measurements of weak-lensing distortion and depletion profiles.

quantitative comparison between different reconstructions will be given in $\S 5$.

\section{MASS PROFILE OF A1689}

In this section, we aim to quantify and characterize the projected mass distribution $(\kappa)$ of A1689 reconstructed from Subaru weak-lensing observations, in order to derive quantitative constraints on the three-dimensional mass distribution. Specifically, we will adopt the NFW density profile $\rho(r) \propto r^{-1}\left(1+r / r_{s}\right)^{-2}$ (Navarro et al. 1997) to describe the cluster mass distribution, characterized by the virial mass $M_{\mathrm{vir}}$ and the concentration parameter $c_{\mathrm{vir}}=r_{\mathrm{vir}} / r_{s}$, defined as the ratio of the virial radius $r_{\text {vir }}$ to the scale radius $r_{s}$. The best-fitting NFW parameters $\left(M_{\mathrm{vir}}, c_{\mathrm{vir}}\right)$ can be then compared with $\Lambda \mathrm{CDM}$ predictions based on $N$-body simulations (Bullock et al. 2001; Neto et al. 2007). As a test for the consistency and reliability, we will compare the best-fitting NFW parameters derived from different combinations of data sets, boundary conditions, and weak-lensing techniques. Here we introduce three different methods to derive a convergence profile $\kappa(\theta)$ from weak-lensing observations. A summary of the methods for mass profile reconstructions is given in Table 3.

\subsection{Method 1: 2D Convergence Map}

The first method makes a direct use of the $2 \mathrm{D} \kappa$ map reconstructed by the entropy-regularized maximum likelihood method ( $(4)$. The $\kappa$ map is directly compared with the model convergence field for an NFW spherical halo specified by two model parameters. We take the virial mass $M_{\mathrm{vir}}$ and the concentration parameter $c_{\text {vir }}$ for describing an NFW halo. We employ the radial dependence of the convergence profile for the NFW model given by Bartelmann (1996). Note that the Bartelmann's formulae for the NFW convergence and shear profiles are obtained assuming the projection integral to extend to infinity. Alternatively, a truncated NFW profile can be used to model the convergence profile (Takada \& Jain 2003). We have confirmed that the best-fitting NFW parameters obtained using the above two different models agree to within 1\% for the case of A1689 lensing (for detailed discussions, see Baltz et al. 2007; Hennawi et al. 2007). We thus simply use Bartlemann's formulae to calculate the relevant lensing fields for an NFW halo as done in $\mathrm{B} 05 \mathrm{a}$ and $\mathrm{B} 05 \mathrm{~b}$.

We constrain the two NFW parameters from $\chi^{2}$ fitting to the 2D convergence map $\kappa(\boldsymbol{\theta})$ derived from Subaru weak-lensing observations. We adopt a flat prior of $c_{\mathrm{vir}} \leq 30$ for the halo concentration because the NFW profiles with $c_{\mathrm{vir}} \gtrsim 20$ cannot be distinguished by the Subaru data alone due to lack of information on the inner density profile (B05a). The $\chi^{2}$-function for the Subaru weak-lensing observations, $\chi^{2}=\chi_{\mathrm{WL}}^{2}$, can be expressed as (Oguri et al. 2005)

$$
\chi_{\mathrm{WL}}^{2}=\sum_{i, j}\left[\hat{\kappa}\left(\boldsymbol{\theta}_{i}\right)-\kappa\left(\boldsymbol{\theta}_{i}\right)\right]\left(C^{-1}\right)_{i j}\left[\hat{\kappa}\left(\boldsymbol{\theta}_{j}\right)-\kappa\left(\boldsymbol{\theta}_{j}\right)\right],
$$

where $\hat{\kappa}\left(\boldsymbol{\theta}_{i}\right)$ is the model prediction of the NFW halo for the $i$ th bin $\left(i=1,2, \ldots, N_{\mathrm{SL}}\right)$, and $\left(C^{-1}\right)_{i j}$ is the inverse of the pixelpixel covariance matrix; $N_{\text {pix }}=31 \times 27=837$. In the model fitting we exclude the central pixel in the strong-lensing region (see $\S 4.4$ ).

We have derived sets of best-fitting NFW parameters for the three different MEM reconstructions listed in Table 2. Table 4 shows a summary of the best-fitting NFW parameters $\left(M_{\mathrm{vir}}, c_{\mathrm{vir}}\right)$ and the resulting $\chi^{2}$ value for our Subaru weak-lensing observations; the errors quote $68 \%$ confidence intervals estimated from $\Delta \chi^{2} \equiv \chi^{2}-\chi_{\min }^{2}=1$ in the $\left(c_{\mathrm{vir}}, M_{\mathrm{vir}}\right)$-plane. Combining the distortion and magnification measurements with the central ACS constraint (MEM+) yields the best-fitting NFW parameters, $M_{\text {vir }}=(1.97 \pm 0.20) \times 10^{15} M_{\odot}$ and $c_{\text {vir }}=13.4_{-3.3}^{+5.4}$, with $\chi_{\min }^{2} / \mathrm{dof}=332 / 834(421)$, where the value in parentheses refers to an effective degrees of freedom excluding upper limit bins with $\kappa<0$. As a test for the consistency, we compare best-fit NFW parameters for the three MEM reconstructions described in $\S 4.7$. Firstly, all of the three MEM reconstructions yield consistent results on the concentration parameter in the range, $c_{\mathrm{vir}}=12.6-$ 13.6, but with rather large uncertainties allowing a wide range of the concentration $\left(8 \lesssim c_{\mathrm{vir}} \lesssim 20\right)$. On the other hand, the best-fit values for $M_{\text {vir }}$ range from $1.47 \times 10^{15} M_{\odot}$ for MEM-S, through $1.62 \times 10^{15} M_{\odot}$ for MEM, to $1.97 \times 10^{15} M_{\odot}$ for MEM+, while the $1 \sigma$ error level for each is $\sigma\left(M_{\text {vir }}\right) \sim 0.2 \times 10^{15} M_{\odot}$.

The observed location of the Einstein radius can be used for a powerful, model independent test of the $\kappa$ profile (B05b). This is based on the fact that the enclosed mass interior to the Einstein radius is given by fundamental constants and with knowledge of distances involved, namely $\bar{\kappa}\left(\theta_{\mathrm{E}}\right)=1$ or $M\left(<\theta_{\mathrm{E}}\right)=$ $\pi\left(D_{d} \theta_{\mathrm{E}}\right)^{2} \Sigma_{\text {crit }}$, provided that the critical curve is nearly circular; this is the case for A1689 (B05b). Although our weak-lensing measurements do not resolve such strong-lensing phenomena, the observed Einstein radius of $\theta_{\mathrm{E}}=45^{\prime \prime}$ (for $z_{s}=1$ ) may be used to test the derived NFW models. To do this we numerically solve the equation $1=\bar{\kappa}_{\mathrm{NFW}}\left(\theta_{\mathrm{E}}\right)$ for the Einstein radius $\theta_{\mathrm{E}}$ by the Newton-Raphson method. We found $\theta_{\mathrm{E}}=36.7_{-18}^{+22}, 36.7_{-20}^{+22}$, and 
TABLE 4

Summary of the Best-Fitting NFW Parameters for Subaru Weak-Lensing Observations

\begin{tabular}{|c|c|c|c|c|c|c|c|}
\hline Method $^{\mathrm{a}}$ & Data & $\mathrm{ACS}^{\mathrm{b}}$ & $\mathrm{ER}^{\mathrm{c}}$ & $M_{\mathrm{vir}}{ }^{\mathrm{d}}$ & $c_{\text {vir }}{ }^{\mathrm{e}}$ & $\chi_{\min }^{2} / \operatorname{dof}^{\mathrm{f}}$ & $\theta_{\mathrm{E}}^{\mathrm{g}}$ \\
\hline \multirow[t]{2}{*}{ Tangential shear ............................. } & $1 \mathrm{D} g_{+}$ & $\ldots$ & $\ldots$ & $1.51_{-0.24}^{+0.27}$ & $20.0_{-5.3}^{+8.8}$ & $5.0 / 8(8)$ & 50.9 \\
\hline & $1 \mathrm{D} g_{+}$ & $\ldots$ & Yes & $1.59_{-0.22}^{+0.24}$ & $15.7_{-2.5}^{+3.4}$ & $9.1 / 9(9)$ & 44.5 \\
\hline \multirow{3}{*}{ 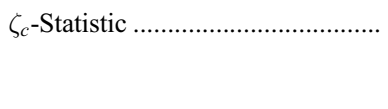 } & $1 \mathrm{D} \kappa$ & $\ldots$ & $\ldots$ & $1.48 \pm 0.27$ & $27.3_{-19.3}^{+2.7}$ & $5.2 / 7(7)$ & 59.1 \\
\hline & $1 \mathrm{D} \kappa$ & $\ldots$ & Yes & $1.51_{-0.22}^{+0.25}$ & $16.5_{-3.1}^{+4.0}$ & $11.4 / 8(8)$ & 44.8 \\
\hline & $1 \mathrm{D} \kappa$ & Yes & $\ldots$ & $1.91_{-0.20}^{+0.24}$ & $13.7_{-1.3}^{+3.1}$ & $12.3 / 19(19)$ & 45.3 \\
\hline \multirow[t]{2}{*}{ 2D MEM-S . } & $2 \mathrm{D} \kappa$ & $\ldots$ & $\ldots$ & $1.48_{-0.18}^{+0.20}$ & $14.1_{-4.8}^{+10.3}$ & $361 / 834(406)$ & 38.2 \\
\hline & $2 \mathrm{D} \kappa$ & Yes & $\ldots$ & $1.75_{-0.16}^{+0.17}$ & $14.6_{-1.1}^{+1.3}$ & $369 / 846(418)$ & 45.1 \\
\hline \multirow[t]{2}{*}{ 2D MEM } & $2 \mathrm{D} \kappa$ & $\ldots$ & $\ldots$ & $1.60_{-0.17}^{+0.21}$ & $14.9_{-5.2}^{+11.9}$ & $287 / 834(478)$ & 42.7 \\
\hline & $2 \mathrm{D} \kappa$ & Yes & $\ldots$ & $1.81_{-0.14}^{+0.11}$ & $14.3_{-1.1}^{+1.2}$ & $294 / 846(490)$ & 45.1 \\
\hline \multirow[t]{3}{*}{ 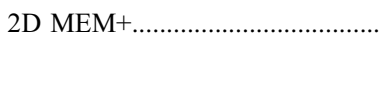 } & $2 \mathrm{D} \kappa$ & $\ldots$ & $\ldots$ & $1.76_{-0.17}^{+0.20}$ & $15.5_{-4.2}^{+7.4}$ & $323 / 836(423)$ & 51.0 \\
\hline & $2 \mathrm{D} \kappa$ & $\ldots$ & Yes & $1.93_{-0.19}^{+0.22}$ & $14.0_{-2.1}^{+2.5}$ & $387 / 837(424)$ & 46.4 \\
\hline & $2 \mathrm{D} \kappa$ & Yes & $\ldots$ & $2.10 \pm 0.17$ & $12.7_{-0.9}^{+1.0}$ & $327 / 848(435)$ & 45.3 \\
\hline \multirow[t]{2}{*}{ Subaru $1 D^{h}$. } & $1 \mathrm{D} \kappa$ & $\ldots$ & $\ldots$ & $1.69_{-0.28}^{+0.30}$ & $\leq 30$ & $5.36 / 8(6)$ & 66.9 \\
\hline & $1 \mathrm{D} \kappa$ & Yes & $\cdots$ & $1.93 \pm 0.20$ & $13.7_{-1.1}^{+1.4}$ & $13.3 / 20(18)$ & 45.4 \\
\hline
\end{tabular}

NoTE.-A flat prior of $c_{\text {vir }} \leq 30$ is adopted in the model fitting.

a Mass reconstruction method (see Table 3).

b Fitting with or without the ACS-derived inner mass profile ( $§$ 5.4.1).

c Fitting with or without the strong-lensing Einstein-radius (ER) constraint, $\theta_{\mathrm{E}}=45^{\prime \prime}$ at $z_{s}=1(\S 5.4 .2)$.

${ }^{\mathrm{d}}$ Virial mass and $1 \sigma$ error in units of $10^{15} M_{\odot}$.

e Concentration parameter, $c_{\mathrm{vir}}=r_{\mathrm{vir}} / r_{s}$, and $1 \sigma$ error.

${ }^{f}$ Values in parentheses refer to effective degrees of freedom excluding upper limit bins with $\kappa<0$.

g Einstein radius in units of arcseconds for a fiducial source at $z_{s}=1$, defined as $1=\bar{\kappa}\left(\theta_{\mathrm{E}}\right)$.

$\mathrm{h}$ Taken from B05a. A fiducial source redshift of $z_{s}=1$ is assumed in B05a.

45. $4_{-15}^{+17}$ for the MEM-S, MEM, and MEM+ reconstructions, respectively (Table 4). All of the MEM reconstructions are roughly consistent with the ACS measurement of the Einstein radius; however, the constraints on the $\theta_{\mathrm{E}}$ placed by the Subaru data alone are still rather weak, naturally due to the lack of central mass distribution.

To make a direct comparison with model predictions, we compute the convergence profile $\kappa(\theta)$ from a weighted radial projection of the $2 \mathrm{D} \kappa$ map as

$$
\kappa_{m} \equiv \kappa\left(\bar{\theta}_{m}\right)=\sum_{i \in \operatorname{bin} m} W_{i m} \kappa\left(\boldsymbol{\theta}_{i}\right) / \sum_{i \in \operatorname{bin} m} W_{i m},
$$

where $W_{\text {im }}\left(0 \leq W_{\text {im }} \leq 1\right)$ is the fraction of the area of the $i$ th pixel lying within the $m$ th annular bin. We use Monte Carlo integration to calculate these area fractions for individual pixels (Marshall et al. 2002), and $\bar{\theta}_{m}$ is the area-weighted center of the $m$ th radial bin:

$$
\theta_{m}=\sum_{i \in \operatorname{bin} m} W_{i m}\left|\boldsymbol{\theta}_{i}\right| / \sum_{i \in \operatorname{bin} m} W_{i m}
$$

Since the $\kappa_{m}$ profile is expressed as a linear combination of $\kappa\left(\boldsymbol{\theta}_{i}\right)$ values, it is straightforward to calculate the covariance matrix $C_{m n} \equiv\left\langle\delta \kappa_{m} \delta \kappa_{n}\right\rangle$ of the reconstruction errors:

$$
\begin{aligned}
C_{m n}= & \sum_{i \in \operatorname{bin} m} \sum_{m \in \operatorname{bin} n} W_{i m} W_{j n} C_{i j}\left(\sum_{i \in \operatorname{bin} m} W_{i m} \sum_{j \in \operatorname{bin} n} W_{j n}\right)^{-1} \\
& +\delta_{m n} \sum_{i \in \operatorname{bin} m} W_{i m}^{2}\left[\kappa_{m}-\kappa\left(\boldsymbol{\theta}_{i}\right)\right]^{2}\left(\sum_{i \in \operatorname{bin} m} W_{i m}\right)^{-2}
\end{aligned}
$$

where the first term represents the errors propagated from the pixel-pixel covariance matrix of the $2 \mathrm{D} \kappa$ reconstruction, and the second term is responsible for variations of the $\kappa(\boldsymbol{\theta})$-field along the azimuthal direction, i.e., departure from circular symmetry.

Here we derive for each of the $2 \mathrm{D} \kappa$ maps a discrete convergence profile (40) in $N_{\text {bin }}$ logarithmically spaced bins for $\theta=$ $\left[\theta_{\min }, \theta_{\max }\right]$; we adopt the same binning as in B05a: $N_{\text {bin }}=10$, $\theta_{\min }=1^{\prime}$, and $\theta_{\max }=18^{\prime}$, as summarized in Table 3 . In Figure 10 we compare the convergence profiles from the 2D MEM+ and MEM reconstructions, both of which are based on the lens distortion and magnification measurements, to clarify the effect of the ACS constraint on the central surface mass density. The error bars represent the $1 \sigma$ uncertainties from the diagonal part $\left(C_{m m}\right)$ of the bin-to-bin covariance matrix given by equation (42), and hence are correlated between the different bins. One can see that overall the convergence profile obtained with the central ACS constraint is slightly steeper than that without the ACS constraint, and has a slightly higher overall normalization; these features are well explained by slightly higher NFW mass $\left(M_{\mathrm{vir}}\right)$ and concentration $\left(c_{\text {vir }}\right)$ derived for the results with the central ACS constraint. Nonetheless, the two convergence profiles are overall in good agreement within the statistical uncertainties. Steep NFW profiles with $c_{\text {vir }} \sim 13$ and $M_{\text {vir }} \sim 2 \times 10^{15} M_{\odot}$ are well fitted to the reconstructed convergence profiles (solid and dashed curves). Figure 11 shows convergence profiles from the three MEM reconstructions: MEM+ (squares), MEM (triangles), and MEM-S (crosses). Note that the vertical axis is linear here rather than logarithmic. The convergence profile from the distortion data alone (MEM-S) shows a slight negative dip of $\kappa \sim-0.01$ at $\theta^{\prime} \gtrsim 6^{\prime}$ due to boundary effects.

\subsection{Method 2: 1D Maximum Likelihood Analysis}

In B05a we developed a method for reconstructing the discrete convergence profile $\kappa_{m}$ from a maximum likelihood combination of radial profiles of the lens distortion and magnification effects on red background galaxies (see Fig. 3 of B05a). With the assumption of quasi-circular symmetry in the projected mass 


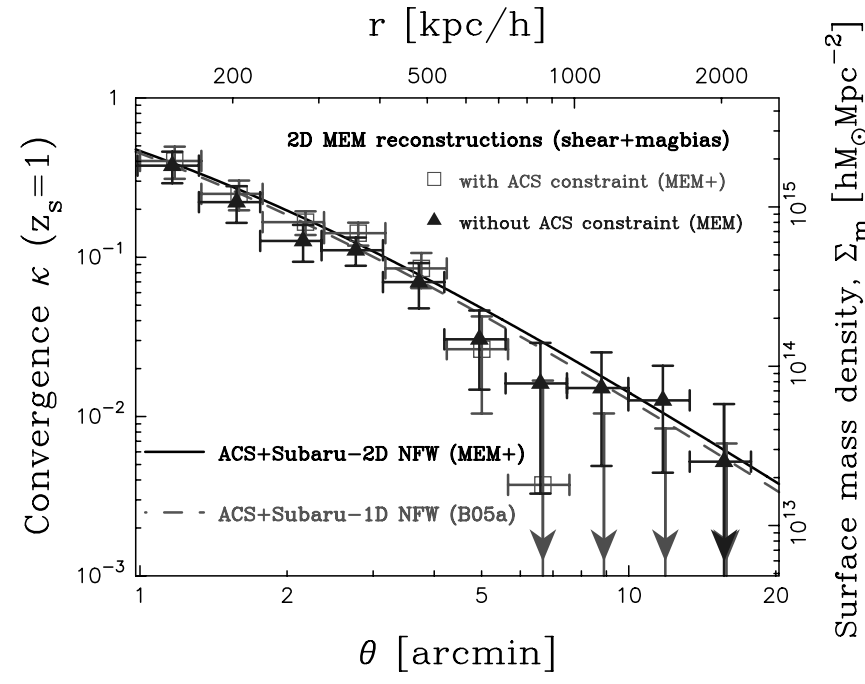

FIG. 10.- Mass profiles of A1689 obtained by a radial projection of the $2 \mathrm{D} \kappa$ map (see Fig. 7) reconstructed from an entropy-regularized maximum likelihood combination of Subaru distortion and depletion measurements. All of the profiles are scaled to a fiducial source redshift of $z_{s}=1$. The square and triangles represent the results with (2D MEM+) and without (2D MEM) ACS strong-lensing constrains on the central mass density, respectively. The error bars are highly correlated in the different bins. Without the central ACS constraint, central $\kappa$-bins at $\theta \lesssim 4^{\prime}$ are slightly underestimated $(\sim 10 \%)$, but the two profiles are overall in good agreement within the statistical uncertainties. The solid curve shows the bestfitting NFW profile for the 2D $\kappa$ map (2D MEM + ) combined with the ACS-derived inner mass profile. For comparison, an NFW model based on the ACS+Subaru $1 \mathrm{D}$ analysis (B05a) is shown as a dashed curve. [See the electronic edition of the Journal for a color version of this figure.]

distribution (or the projected potential field), one can express the lensing observables (i.e., tangential distortion and magnification bias) in terms of the binned convergence profile (see also $\S 5.3$ ). Owing to the nature of shear-based reconstruction, boundary conditions need to be specified (as discussed in $\S 4.7$ ). In B05a we set the inner boundary condition on the mass interior to $\theta_{\min }=1^{\prime}$, which is readily obtained from the well-constrained ACS mass model.

One of the advantages of such a 1D method is that one can improve on the statistical significance of each measurement pixel by azimuthal averaging, provided that the system is nearly symmetric. B05a measured from the red background sample a tangential distortion and a radial depletion profile in 10 discrete radial bins, with total significance of $\mathrm{S} / \mathrm{N} \simeq 14.2$ and $=9.2$, respectively (see $\S 4.7$ ). Thus, the per pixel $\mathrm{S} / \mathrm{N}$ of each measurement is of the order of unity, which is optimal for an inversion problem. Accordingly, the $1 \mathrm{D}$ analysis does not require regularization techniques. The best-fitting NFW parameters for the Subaru 1D analysis are listed in Table 2.

\subsection{Method 3: Aperture Densitometry}

The spin-2 shape distortion of an object due to gravitational lensing is described by the complex reduced shear, $g=g_{1}+i g_{2}$ (see eq. [10]), which is coordinate dependent. For a given reference point on the sky, one can instead form coordinate-independent quantities, the tangential distortion $g_{+}$and the $45^{\circ}$ rotated component, from linear combinations of the distortion coefficients $g_{1}$ and $g_{2}$ as

$$
\begin{aligned}
g_{+} & =-\left(g_{1} \cos 2 \phi+g_{2} \sin 2 \phi\right), \\
g_{\times} & =-\left(g_{2} \cos 2 \phi-g_{1} \sin 2 \phi\right),
\end{aligned}
$$

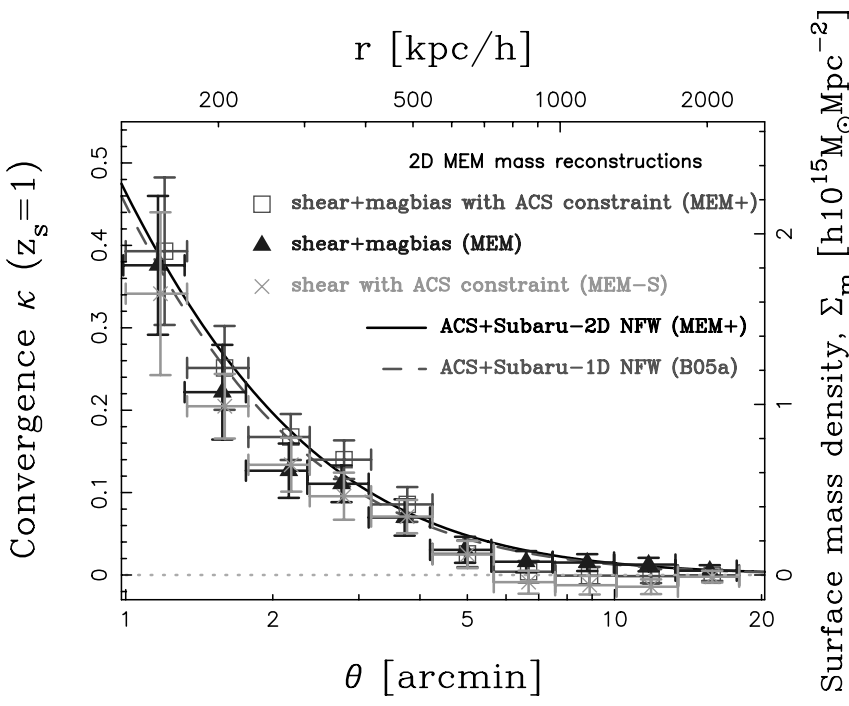

FIG. 11.-Comparison of mass profiles from MEM-reconstructed $\kappa$ maps based on different combinations of Subaru data sets and boundary conditions. All of the profiles are scaled to a fiducial source redshift of $z_{s}=1$. The square and triangles represent the results from the combined distortion and depletion measurements, with (MEM+) and without (MEM) the ACS constraint on the mean surface mass density in the central pixel, respectively. The crosses show the results from the distortion data with the central ACS constraint (MEM-S). The model curves are shown for comparison as in Fig. 10. The mass profile from the distortion data alone (crosses) shows a slight negative dip of $\kappa \sim-0.01$ at $6^{\prime} \lesssim \theta \lesssim 10$ due to spurious boundary effects. [See the electronic edition of the Journal for a color version of this figure.]

where $\phi$ is the position angle of an object with respect to the reference position, and the uncertainty in the $g_{+}$and $g_{\times}$measurement is $\sigma_{+}=\sigma_{\times}=\sigma_{g} / \sqrt{2} \equiv \sigma$ in terms of the rms error $\sigma_{g}$ for the complex shear measurement. In practice, the reference point is taken to be the cluster center, which is well determined from symmetry of the strong-lensing pattern. To improve the statistical significance of the distortion measurement, we calculate the weighted average of the $g_{+}$values and its weighted error as

$$
\begin{aligned}
\left\langle g_{+}(\theta)\right\rangle & =\frac{\sum g_{+} / \sigma^{2}}{\sum 1 / \sigma^{2}}, \\
\sigma_{+}(\theta) & =\left(\sum 1 / \sigma^{2}\right)^{-1 / 2} .
\end{aligned}
$$

For a shear-based estimation of the cluster mass profile we use a variant of the weak-lensing aperture densitometry, or the so-called $\zeta$-statistic (Fahlman et al. 1994; Clowe et al. 2000), of the form

$$
\begin{aligned}
\zeta_{c}(\theta) \equiv & 2 \int_{\theta}^{\theta_{\text {inn }}} d \ln \theta^{\prime} \gamma_{+}\left(\theta^{\prime}\right) \\
& +\frac{2}{1-\left(\theta_{\text {inn }} / \theta_{\text {out }}\right)^{2}} \int_{\theta_{\text {inn }}}^{\theta_{\text {out }}} d \ln \theta^{\prime} \gamma_{+}\left(\theta^{\prime}\right) \\
= & \bar{\kappa}(\theta)-\bar{\kappa}\left(\theta_{\text {inn }}<\vartheta<\theta_{\text {out }}\right),
\end{aligned}
$$

where $\bar{\kappa}(\theta)$ is the average convergence interior to radius $\theta$, and $\theta_{\text {inn }}$ and $\theta_{\text {out }}$ are the inner and outer radii of the annular background region in which the mean background contribution, $\bar{\kappa}_{b} \equiv \bar{\kappa}\left(\theta_{\text {inn }}<\right.$ $\vartheta<\theta_{\text {out }}$ ), is defined; the $\gamma_{+}(\theta)=\bar{\kappa}(\theta)-\kappa(\theta)$ is an azimuthal average of the tangential component of the gravitational shear at radius $\theta$ (Fahlman et al. 1994), which is observable in the 
weak-lensing limit: $\gamma_{+}(\theta) \approx\left\langle g_{+}(\theta)\right\rangle$. This cumulative mass estimator subtracts from the mean convergence $\bar{\kappa}(\theta)$ a constant $\bar{\kappa}_{b}$ for all apertures $\theta$ in the measurements, thus removing any DC component in the control region $\theta=\left[\theta_{\text {inn }}, \theta_{\text {out }}\right]$. We note that the $\bar{\kappa}_{b}$ is a nonobservable free parameter, and we use this degree of freedom to fix the outer boundary condition, and hence to derive a convergence profile.

We compute the aperture densitometry profile $\zeta_{c}\left(\theta_{m}\right)$ in $N_{\zeta}=10$ logarithmically spaced bins for $\theta=\left[\theta_{\min }, \theta_{\max }\right]$, which we set to $\theta_{\min }=1^{\prime}$ and $\theta_{\max }=16^{\prime}$; the maximum radius $\theta_{\max }$ is comparable to the angular size of the cluster virial radius, $\theta_{\mathrm{vir}}=$ $15.7^{\prime}\left(r_{\text {vir }} \sim 2 \mathrm{Mpc} h^{-1}\right)$, according to the ACS+Subaru-1D model (B05a). The inner and outer background radii are set to $\theta_{\text {inn }}=\theta_{\text {max }}=16^{\prime}$ and $\theta_{\text {out }}=19^{\prime}$, respectively. Using the ACS + Subaru-1D model by B05a, the mean background level, $\bar{\kappa}_{b}$, is calculated to be $\bar{\kappa}_{b} \sim 4.0 \times 10^{-3}$ for our red background population with $\left\langle D_{d s} / D_{s}\right\rangle=0.693$, at an effective source redshift of $z_{s, D}=0.68$ (see eq. [18]). Note that the current $1 \sigma$ upper limit is $\kappa(\theta) \lesssim 0.01$ at $\theta \gtrsim 8^{\prime}(\mathrm{B} 05 \mathrm{a})$. For a given boundary condition $\bar{\kappa}_{b}$, the average convergence $\bar{\kappa}\left(\theta_{m}\right)$ is estimated as

$$
\bar{\kappa}\left(\theta_{m}\right)=\zeta_{c}\left(\theta_{m}\right)+\bar{\kappa}_{b}
$$

Then, we define a discretized estimator for $\kappa$ as

$$
\kappa_{m} \equiv \kappa\left(\bar{\theta}_{m}\right)=\alpha_{2}^{m} \zeta\left(\theta_{m+1}\right)-\alpha_{1}^{m} \zeta\left(\theta_{m}\right)+\left(\alpha_{2}^{m}-\alpha_{1}^{m}\right) \bar{\kappa}_{b},
$$

where

$$
\begin{aligned}
& \alpha_{1}^{m}=\frac{1}{2 \Delta \ln \theta_{m}}\left(\frac{\theta_{m}}{\bar{\theta}_{m}}\right)^{2}, \\
& \alpha_{2}^{m}=\frac{1}{2 \Delta \ln \theta_{m}}\left(\frac{\theta_{m+1}}{\bar{\theta}_{m}}\right)^{2},
\end{aligned}
$$

and $\bar{\theta}_{m}$ is the weighted center of the $m$ th radial bin $(m=$ $1,2, \ldots, N_{\zeta}-1$; see Appendix A). It is worth noting that, unlike strong-lensing based boundary conditions (e.g., B05a), this method utilizes an outer boundary condition on the mean background density $\bar{\kappa}_{b}$ to derive a $\kappa$ profile (see Schneider \& Seitz 1995 for an alternative method for a direct inversion of the mass profile). The error covariance matrix $C_{m n}$ of $\kappa_{m}$ is expressed as

$$
\begin{aligned}
C_{m n} \equiv\left\langle\delta \kappa_{m} \delta \kappa_{n}\right\rangle & =\alpha_{2}^{m} \alpha_{2}^{n} C_{m+1, n+1}^{\zeta}+\alpha_{1}^{m} \alpha_{1}^{n} C_{m, n}^{\zeta} \\
& -\alpha_{1}^{m} \alpha_{2}^{n} C_{m, n+1}^{\zeta}-\alpha_{2}^{m} \alpha_{1}^{n} C_{m+1, n}^{\zeta}
\end{aligned}
$$

where $C_{m n}^{\zeta} \equiv\left\langle\delta \zeta_{m} \delta \zeta_{n}\right\rangle$ is the bin-to-bin error covariance matrix of the aperture densitometry measurements which is calculated by propagating the rms errors $\sigma_{+}\left(\theta_{m}\right)$ for the tangential shear measurement (Okabe \& Umetsu 2008).

In the nonlinear regime, however, the $\gamma_{+}(\theta)$ is not a direct observable. Therefore, nonlinear corrections need to be taken into account in the mass reconstruction process. In the subcritical regime (i.e., outside the critical curves), the $\gamma_{+}(\theta)$ can be expressed in terms of the averaged tangential reduced shear as $\left\langle g_{+}(\theta)\right\rangle \approx \gamma_{+}(\theta) /[1-\kappa(\theta)]$ assuming a quasi circular symmetry in the projected mass distribution (B05a; Umetsu et al. 2007). This nonlinear equation (46) for $\zeta_{c}(\theta)$ can be solved by an iterative procedure: since the weak-lensing limit $(\kappa,|\gamma|,|g| \ll 1)$

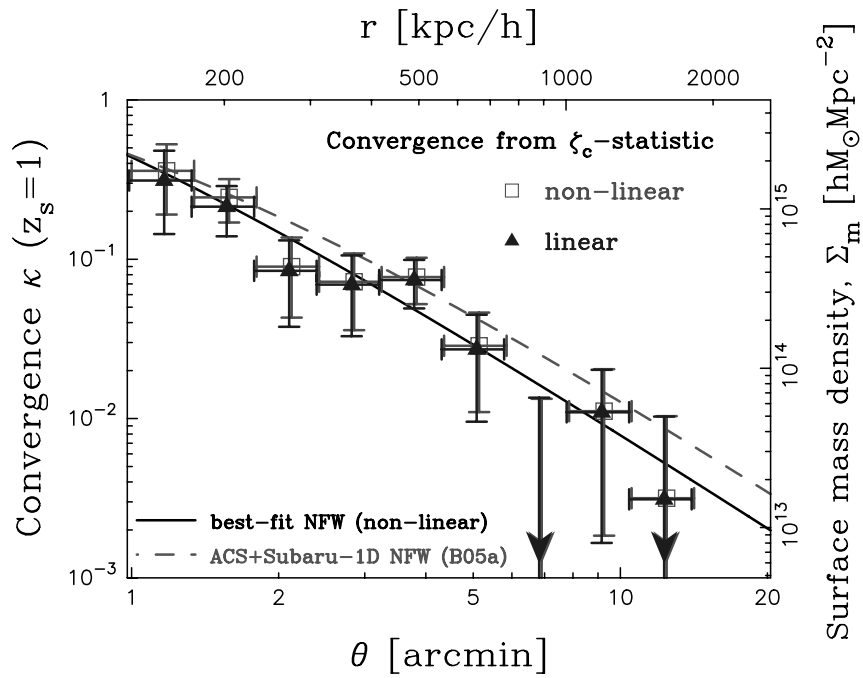

FIG. 12.- Shear-based 1D mass reconstruction utilizing the $\zeta_{c}$-statistic. As an outer boundary condition, the mean background density $\bar{\kappa}_{b}$ in the range $16^{\prime} \lesssim \theta \lesssim 19^{\prime}$ is set to $\bar{\kappa}_{b}=4 \times 10^{-3}$ according to the ACS+Subaru-1D best-fit NFW model (B05a). The squares represent the results with the nonlinear corrections. The triangles show the reconstruction with linear approximation. The mass profiles are scaled to a fiducial source redshift of $z_{s}=1$. Decorrelated error bars are shown. Downward-pointing arrows are used where the lower error bar drops below zero. Without the nonlinear corrections, central bins are underestimated by $\sim 15 \%$ at maximum. The solid curve shows an NFW profile with a high concentration, $c_{\mathrm{vir}}=30$, matching well the overall profile obtained with the nonlinear corrections. For comparison an NFW model based on the combined ACS and Subaru distortion and depletion profiles (B05a) is shown as a dashed curve. [See the electronic edition of the Journal for a color version of this figure.]

holds in the background region $\theta_{\text {inn }} \leq \theta \leq \theta_{\max }$, we have the following iterative equation for $\zeta_{c}(\theta)$ :

$$
\begin{aligned}
\zeta_{c}^{(k+1)}(\theta) \approx & 2 \int_{\theta}^{\theta_{\text {inn }}} d \ln \theta^{\prime}\left\langle g_{+}(\theta)\right\rangle\left[1-\kappa^{(k)}(\theta)\right] \\
& +\frac{2}{1-\left(\theta_{\text {inn }} / \theta_{\text {out }}\right)^{2}} \int_{\theta_{\text {inn }}}^{\theta_{\text {out }}} d \ln \theta^{\prime}\left\langle g_{+}\left(\theta^{\prime}\right)\right\rangle,
\end{aligned}
$$

where $\zeta_{c}^{(k+1)}$ represents the aperture densitometry in the $(k+1)$ th step of the iteration $\left(k=0,1,2, \ldots, N_{\text {iter }}\right)$; the $\kappa^{(k+1)}$ is calculated from $\zeta_{c}^{(k+1)}$ using equation (48). This iteration is performed by starting with $\kappa^{(0)}=0$ for all radial bins, and repeated until convergence is reached at all radial bins. For a fractional tolerance of $1 \times 10^{-5}$, this iteration procedure converges within $N_{\text {iter }} \sim 10$ iterations. We compute errors for $\zeta_{c}$ and $\kappa$ with the linear approximation.

Figure 12 shows the resulting model-independent mass profile (squares) of A1689 with decorrelated error bars, along with the results without the nonlinear corrections (triangles). Without the nonlinear corrections, central bins at $\theta \lesssim 3^{\prime}$ are underestimated by $\sim 15 \%$ at maximum. The reconstructed convergence in the first bin is $\kappa\left(1.2^{\prime}\right)=0.32 \pm 0.15$ (decorrelated $1 \sigma$ error), which is consistent at the $\sim 1 \sigma$ level with the previous Subaru 1D results (B05a): $\kappa\left(1.2^{\prime}\right)=0.44_{-0.12}^{+0.11}(1 \sigma)$ for the red background population with $\left\langle D_{d s} / D_{s}\right\rangle \approx 0.693$. We note that B05a utilized an inner boundary condition on the mean $\kappa$ interior to $\theta_{\min }=1^{\prime}$ based on the ACS mass profile.

Force fitting an NFW profile to the derived $\kappa$ profile yields $M_{\text {vir }}=(1.48 \pm 0.27) \times 10^{15} M_{\odot}$ and $c_{\text {vir }}=27.3_{-19.3}^{+2.7}$ with the adopted prior $c_{\mathrm{vir}} \leq 30$, where $\chi_{\mathrm{min}}^{2} / \mathrm{dof}=5.2 / 7(7)$. It is interesting to compare the reconstructed $\kappa$ profile with the tangential shear profile, $\left\langle g_{+}(\theta)\right\rangle$, which is a direct observable with uncorrelated measurement errors (see eq. [44]). In B05a we show the 
tangential distortion profile for the same red background sample as used in the present study (see Fig. 1 of B05a, or Fig. 16, below). The best-fitting NFW model for the shear profile is given by $M_{\mathrm{vir}}=(1.51 \pm 0.26) \times 10^{15} M_{\odot}$ and a high concentration, $c_{\mathrm{vir}}=20_{-5.3}^{+8.8}\left(\chi_{\min }^{2} / \mathrm{dof}=5.0 / 8\right)$, which is in good agreement with the results from the 1D reconstruction based on the aperture densitometry. Such a detailed agreement ensures the validity of the boundary condition for a shear-based mass reconstruction. We note that simply assuming $\bar{\kappa}_{b}=0$ yields similar results, $M_{\mathrm{vir}}=$ $(1.51 \pm 0.3) \times 10^{15} M_{\odot}\left(c_{\mathrm{vir}} \leq 30\right)$ with $\chi_{\min }^{2} / \mathrm{dof}=5.5 / 7(5)$, being consistent within the $1 \sigma$ uncertainty.

\subsection{Combining Strong- and Weak-Lensing Results}

\subsubsection{ACS Constraints}

As demonstrated in $\mathrm{B} 05 \mathrm{a}$ and the previous sections, it is crucial to have information on the central mass distribution in order to derive useful constraints on the concentration parameter, and hence to distinguish the NFW profile from others. To do this, we constrain the two NFW parameters from $\chi^{2}$-fitting to the combined Subaru weak-lensing and ACS strong-lensing observations following B05a and Oguri et al. (2005):

$$
\chi^{2}=\chi_{\mathrm{WL}}^{2}+\chi_{\mathrm{SL}}^{2}
$$

where the $\chi_{\mathrm{WL}}^{2}$ for the Subaru observations is defined by equation (39). For the ACS data we use an azimuthally averaged profile of the $\kappa$ map well constrained by the strong-lensing observations of $\mathrm{B} 05 \mathrm{~b}$; this profile is given in $N_{\mathrm{SL}}=12$ bins linearly spacing over the range $\theta=\left[0.077^{\prime}, 0.97^{\prime}\right]$ (see Fig. 22 of B05b, or Fig. 3 of B05a), and the amplitude is scaled to $\left\langle D_{d s} / D_{s}\right\rangle=0.693$ of our red background sample from $D_{d s} / D_{s}=$ 0.881 at $z_{s}=3$. It is important to note that the ACS stronglensing analysis of B05b unveiled the secondary mass clump associated with a small clump of galaxies (Teague et al. 1990; Czoske 2004). However, the mass contribution of this subclump is only a small fraction of the cluster mass component (see Figs. 21 and 22 of B05b), and it only slightly perturbs the tangential critical curve (see B05b). The ACS mass profile is corrected for the subclump, where the secondary clump region is locally masked out when taking an azimuthal average (B05b). By combining the Subaru and ACS lensing analyses, we can trace the cluster mass distribution over a large range in amplitude $\kappa \sim\left[10^{-3}, 1\right]$ and in projected radius $r=\left[10^{-2}, 2\right] \mathrm{Mpc} h^{-1}$. The $\chi^{2}$ function for the ACS observations is expressed as

$$
\chi_{\mathrm{SL}}^{2}=\sum_{i=1}^{N_{\mathrm{SL}}} \frac{\left(\kappa_{i}-\hat{\kappa}_{i}\right)^{2}}{\sigma_{i}^{2}},
$$

where $\hat{\kappa}\left(\boldsymbol{\theta}_{i}\right)$ is the model prediction of the NFW halo for the $i$ th pixel $\left(i=1,2, \ldots, N_{\text {pix }}\right)$, and $\sigma_{i}$ is the $1 \sigma$ error for $\kappa_{i}$; the bin width of the ACS-derived convergence profile is sufficiently broad to ensure that the errors between different bins are independent (B05b).

\subsubsection{Einstein-Radius Constraint}

Alternatively, as a model-independent constraint, ${ }^{8}$ we can utilize the observed location of tangential critical curves traced by giant arcs and multiply imaged background galaxies, defining an approximate Einstein radius, $\theta_{\mathrm{E}}$. This radius is determined

\footnotetext{
${ }^{8}$ We note that this is based on the assumption of the circular symmetry in the projected lens mass distribution, and that a rather tight prior (estimated from data) is applied to one of the "parameters" (i.e., $\theta_{\mathrm{E}}$ ).
}

from strong-lensing modeling of many multiple images visible around these clusters in deep HST ACS images (B05b) and corresponds to the theoretically extreme value of the ellipticity, $g_{+}\left(\theta_{\mathrm{E}}\right)=1$. This Einstein-radius constraint complements in a model-independent manner the weak-lensing shape measurements.

We constrain the NFW model parameters $\left(c_{\mathrm{vir}}, M_{\mathrm{vir}}\right)$ by combining weak-lensing profiles and the Einstein-radius constraint. We define the $\chi^{2}$-function for combined weak-lensing distortion and Einstein-radius constraints by

$$
\chi^{2}=\sum_{m} \frac{\left[\left\langle g_{+}\left(\theta_{m}\right)\right\rangle-\hat{g}_{+}\left(\theta_{m}\right)\right]^{2}}{\sigma_{+}^{2}\left(\theta_{m}\right)}+\frac{\left[1-\hat{g}_{+}\left(\theta_{\mathrm{E}}, z_{\mathrm{E}}\right)\right]^{2}}{\sigma_{+, \mathrm{E}}^{2}},
$$

where the first term is the $\chi^{2}$-function for the Subaru tangential shear measurements $(\S 5.3)$ and the second term is the $\chi^{2}$ function for the Einstein radius constraint; $\hat{g}_{+}\left(\theta_{m}\right)$ is the NFW model prediction for the reduced tangential shear at $\theta=\theta_{m}$ calculated for the red background sample (see $\S 3.3), \hat{g}_{+}\left(\theta_{\mathrm{E}}, z_{\mathrm{E}}\right)$ is the model prediction for the reduced tangential shear at $\theta=\theta_{\mathrm{E}}$, evaluated at the arc redshift, $z_{s}=z_{\mathrm{E}}$, and $\sigma_{+, \mathrm{E}}$ is the rms error for $g_{+}\left(\theta_{\mathrm{E}}, z_{\mathrm{E}}\right)$. Following B05b, we take $\theta_{\mathrm{E}}=45^{\prime \prime}$ at $z_{E}=1$, and assume conservatively a $10 \%$ error for the Einstein radius: $\sigma_{\theta_{\mathrm{E}}} / \theta_{\mathrm{E}}=0.1$. We then propagate this error to $g_{+}$assuming a singular isothermal sphere (SIS) model. At $r \lesssim r_{s}$, the density slope of NFW is shallower than that of SIS (see Fig. 1 of Wright $\&$ Brainerd 2000), so that this gives a fairly conservative estimate of $\sigma_{+, \mathrm{E}}$. For the SIS model, $\partial \ln g_{+} / \partial \ln \theta_{\mathrm{E}}=2$ at $\theta=\theta_{\mathrm{E}}$, so that $\sigma_{+, \mathrm{E}}=2 \sigma_{\theta_{\mathrm{E}}} / \theta_{\mathrm{E}}=0.2$.

Alternatively we can combine the $\kappa$ profile reconstructed from weak-lensing distortion measurements ( $(5.3)$ with the Einsteinradius constraint. For an axially symmetric lens, the Einsteinradius constraint is simply written as $\bar{\kappa}\left(\theta_{\mathrm{E}}, z_{\mathrm{E}}\right)=1$. Thus, the $\chi^{2}$-function is now expressed as

$$
\begin{aligned}
\chi^{2}= & \sum_{m, n}\left[\kappa\left(\theta_{m}\right)-\hat{\kappa}\left(\theta_{m}\right)\right]\left(C^{-1}\right)_{m n}\left[\kappa\left(\theta_{n}\right)-\hat{\kappa}_{n}\left(\theta_{n}\right)\right] \\
& +\frac{\left[1-\hat{\bar{\kappa}}\left(\theta_{\mathrm{E}}, z_{\mathrm{E}}\right)\right]^{2}}{\sigma_{\bar{\kappa}, \mathrm{E}}^{2}}
\end{aligned}
$$

where $\hat{\bar{\kappa}}\left(\theta_{\mathrm{E}}, z_{\mathrm{E}}\right)$ is the model prediction for the average convergence interior to the Einstein radius $\theta_{\mathrm{E}}$ evaluated at the arc redshift of $z_{s}=z_{\mathrm{E}}$, and $\sigma_{\bar{\kappa}, \mathrm{E}}$ is the rms error for $\bar{\kappa}\left(\theta_{\mathrm{E}}, z_{\mathrm{E}}\right)$, which we take as $\sigma_{\bar{\kappa}, \mathrm{E}}=\sigma_{\theta_{\mathrm{E}}} / \theta_{\mathrm{E}}=0.1$.

\subsubsection{Comparison}

Table 4 summarizes for each mass reconstruction the bestfitting NFW parameters $\left(M_{\mathrm{vir}}, c_{\mathrm{vir}}\right)$, the minimized $\chi^{2}$-value with respect to the degrees of freedom, and the predicted Einstein radius $\theta_{\mathrm{E}}$ for a fiducial source at $z_{s}=1$. Here for each of the Subaru reconstructions, we compare the best-fit NFW parameters with and without the ACS inner profile combined, as indicated in the third column of Table 4. When combined with the inner ACS profile, all of the Subaru reconstructions yield a virial mass in the range $M_{\mathrm{vir}} \sim(1.8-2.1) \times 10^{15} M_{\odot}$ and a high concentration in the range $c_{\mathrm{vir}} \sim 13-15$, properly reproducing the observed Einstein radius of $\sim 45^{\prime \prime}$ at $z_{s}=1$ (B05b). In particular, fitting an NFW profile to the combined ACS and Subaru-2D data based on the MEM+ mass reconstruction (see Table 2) yields $M_{\mathrm{vir}}=(2.10 \pm 0.17) \times 10^{15} M_{\odot}$ and $c_{\mathrm{vir}}=12.7_{-0.9}^{+1.0}\left(\chi_{\min }^{2} / \mathrm{dof}=\right.$ 335846; effective dof of 433 without including upper limit bins with $\kappa<0$ ), with the predicted Einstein radius of $\theta_{\mathrm{E}}=45.3_{-6.2}^{+5.9} \mathrm{arcsec}$. 


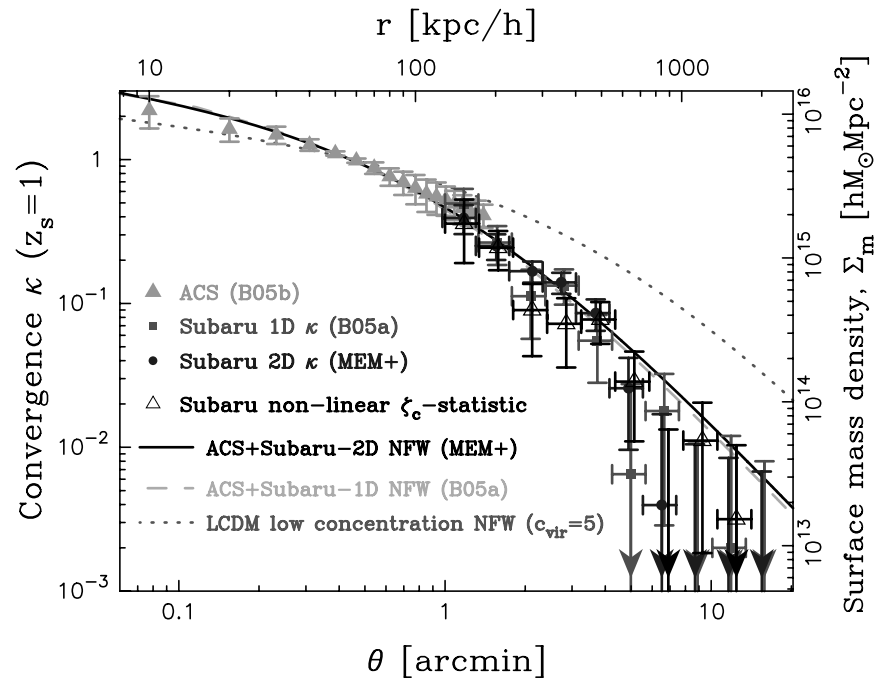

FIG. 13.-Comparison of model-independent mass profiles of A1689. All of the profiles are scaled to a fiducial source redshift of $z_{s}=1$. The filled circles represent the results based on the $2 \mathrm{D} \kappa$ map reconstructed from an entropyregularized maximum likelihood combination of Subaru distortion and depletion data, with the ACS constraint on the mean surface mass density in the central pixel $(\mathrm{MEM}+)$. The error bars are correlated. The open triangles represent the mass profile from the nonlinear $\zeta_{c}$-statistic measurements based on averaged tangential distortion data. Decorrelated error bars are shown. The filled triangles and circles show the results from the ACS strong-lensing analysis (B05b) and from the Subaru 1D weak-lensing analysis based on the combined distortion and depletion profiles (B05a). The solid curve shows the best-fitting NFW profile for the MEM-reconstructed 2D $\kappa$ map (MEM+). The 1D- and 2D-based NFW models from the respective combined ACS+Subaru data (B05a) are also shown as solid and dashed curves, respectively. For comparison an NFW profile with a low concentration, $c_{\mathrm{vir}}=5$, normalized to the observed Einstein radius $\left(\theta_{\mathrm{E}}=45^{\prime \prime}\right)$, is shown as a dotted curve. The low-concentration model (dotted curve) predicted for $\Lambda$ CDM clearly overestimates the outer profile constrained by the Subaru weak-lensing observations. The mass profiles are all in remarkable agreement over the full range of radii up to $\sim 2 \mathrm{Mpc} h^{-1}$. [See the electronic edition of the Journal for a color version of this figure.]

The 2D-based results here can be compared with the corresponding results from the Subaru 1D analysis of B05a (see Table 4) based on the combined distortion and magnification measurements. The combined ACS and Subaru-1D convergence profile is well fitted by an NFW profile with $M_{\text {vir }}=(1.9 \pm 0.2) \times$ $10^{15} M_{\odot}$ and $c_{\mathrm{vir}}=13.7_{-1.1}^{+1.4}$ (B05a), with the predicted Einstein radius of $\theta_{\mathrm{E}}=45.4_{-6.9}^{+7.6}$ arcsec, in good agreement with the present full 2D analysis within the statistical uncertainties; this agreement between the 1D and 2D analyses supports the assumption of quasi-circular symmetry in the projected mass distribution. It is interesting to compare these results with different combinations of lensing measurements having different systematics. Another combination of the ACS and Subaru 1D convergence profile, derived from the shear-based $\zeta$-statistic measurements ( $\S 5.4 .2$ ), yields fairly consistent results: $M_{\mathrm{vir}}=1.91_{-0.20}^{+0.24} \times$ $10^{15} M_{\odot}$ and $c_{\text {vir }}=13.7_{-1.3}^{+1.5}$. This consistency clearly demonstrates that our results here are insensitive to systematic errors in the lensing measurements, such as the shear calibration error.

In Figure 13 we make a direct comparison between modelindependent convergence profiles of A1689 from different weaklensing techniques, along with the ACS-derived inner profile obtained by $\mathrm{B} 05 \mathrm{~b}$ ( filled triangles). The filled circles with error bars show the results based on the $2 \mathrm{D} \kappa$ map reconstructed from an entropy-regularized maximum likelihood combination of the lens magnification and distortion of red background galaxies (MEM + , Table 2). The open triangles represent the $\kappa$ profile from the nonlinear $\zeta_{c}$-statistic measurements. The filled circles show the results from the Subaru 1D analysis by B05a based on the combined magnification and distortion profiles of the same red background population as in the present study. It is clearly seen from Figure 13 that the Subaru-derived $\kappa$ profiles are all in good agreement within the statistical uncertainties, over the full range of radii up to $\sim 2 \mathrm{Mpc} h^{-1}$. The entire mass profile traced by the combined ACS and Subaru information is well described by a single NFW profile (solid and dashed lines) with a high concentration $\left(c_{\mathrm{vir}} \sim 13-14\right)$. For comparison an NFW profile with a low concentration $c_{\mathrm{vir}}=5$ normalized to the observed Einstein radius of $45^{\prime \prime}$ (at $z_{s}=1$ ) is shown as a dotted curve. Such a lowconcentration profile as favored by the standard $\Lambda$ CDM model clearly overpredicts the outer profile constrained by the Subaru weak-lensing observations.

Figure 14 shows the $68 \%, 95 \%$, and $99.7 \%$ confidence levels $\left(\Delta \chi^{2}=2.3,6.17\right.$, and 11.8) in the $\left(c_{\mathrm{vir}}, M_{\mathrm{vir}}\right)$-plane for each of the three different 2D MEM reconstructions (from top to bottom: MEM-S, MEM, and MEM+; see Table 2) with (right) and without (left) the central ACS profile at $10 \mathrm{kpc} h^{-1} \lesssim r \lesssim 180 \mathrm{kpc} h^{-1}$ combined. As shown in the left panels, $M_{\mathrm{vir}}$ is well constrained by the Subaru data alone, while the Subaru constraint on $c_{\text {vir }}$ is rather weak, allowing a wide range of the concentration parameter, $c_{\mathrm{vir}}$. The complementary ACS observations, when combined with the Subaru observations, significantly narrow down the uncertainties on $c_{\text {vir }}$ (right), placing stringent constraints on the inner mass profile. For each subpanel, the observed constraints on the Einstein radius, $\theta_{\mathrm{E}} \simeq 45^{\prime \prime}$ at $z_{s}=1$, are shown as a dashed curve. This clearly demonstrates that the ACS-derived constraints on the $\kappa$ field ensure the correct size of the observed Einstein radius.

Table 5 lists the best-fitting NFW parameters obtained in different studies. Here we quote as the NFW parameters $\left(c_{200}, M_{200}\right)$ evaluated at a specific fractional overdensity of $\Delta_{c}=200$ with respect to the critical density $\rho_{\text {crit }}\left(z_{d}\right)$ for closure of the universe at $z_{d}=0.183$ as well as those in terms of the virial properties, $\left(c_{\mathrm{vir}}, M_{\mathrm{vir}}\right)$. We convert a given set of $\left(c_{200}, M_{200}\right)$ into $\left(c_{\mathrm{vir}}, M_{\mathrm{vir}}\right)$ assuming a spherical NFW density profile (see, e.g., Appendix A of Shimizu et al. 2003). We show in Figure 15 the same confidence levels as in Figure 14 but for the Subaru $g_{+}$profile of B05a. Also shown are best-fit sets of $\left(c_{\mathrm{vir}}, M_{\mathrm{vir}}\right)$ taken from Halkola et al. (2006) and Limousin et al. (2007) as well as from our combined ACS and MEM+ results. The best-fitting NFW parameters for the Subaru $g_{+}$profile (cross) are $M_{\mathrm{vir}}=1.51_{-0.24}^{+0.27} \times 10^{15} M_{\odot}$ and $c_{\text {vir }}=20.0_{-5.3}^{+8.8}$ (Table 4), being marginally consistent with the very high concentration of $c_{\mathrm{vir}}=27.2_{-5.7}^{+3.5}$ derived by Medezinski et al. (2007). ${ }^{9}$ The $\kappa$ field derived from the present full 2D analysis, in conjunction with the central ACS profile, favors a slightly lower, but still high, concentration of $c_{\mathrm{vir}} \sim 13$ (triangle), reproducing the observed Einstein radius of $\sim 45^{\prime \prime}$ at $z_{s}=1$. On the other hand, Limousin et al. (2007) found from their CFHT weak-lensing analysis a concentration of $c_{\text {vir }}=9.6 \pm 2.0$ and a virial mass of $M_{\mathrm{vir}}=1.5_{-0.2}^{+0.3} \times 10^{15} M_{\odot}$ (filled circle $)$, corresponding to the Einstein radius of $24^{\prime \prime} \pm 11^{\prime \prime}$ (at $z_{s}=1$ ), inconsistent with the observed Einstein radius. The results from Halkola et al. (2006) with $c_{\text {vir }}=9.6 \pm 0.4$ and $M_{\text {vir }}=2.6_{-0.1}^{+0.2} \times$ $10^{15} M_{\odot}$ (square) come closer to the observed Einstein radius, being marginally consistent within the $1 \sigma$ uncertainty $\left(\theta_{\mathrm{E}}=\right.$ $39_{-3}^{+6} \operatorname{arcsec}$ at $\left.z_{S}=1\right)$, based on almost exactly the same shear data used in B05a but with a different weighting which prefers their inner strong-lensing based profile where the ACS data

\footnotetext{
${ }^{9}$ Unlike our analysis here, Medezinski et al. (2007) used the observed constraints on the Einstein radius when fitting an NFW profile to the $g_{+}$profile measured from the combined red and blue sample of the background.
} 

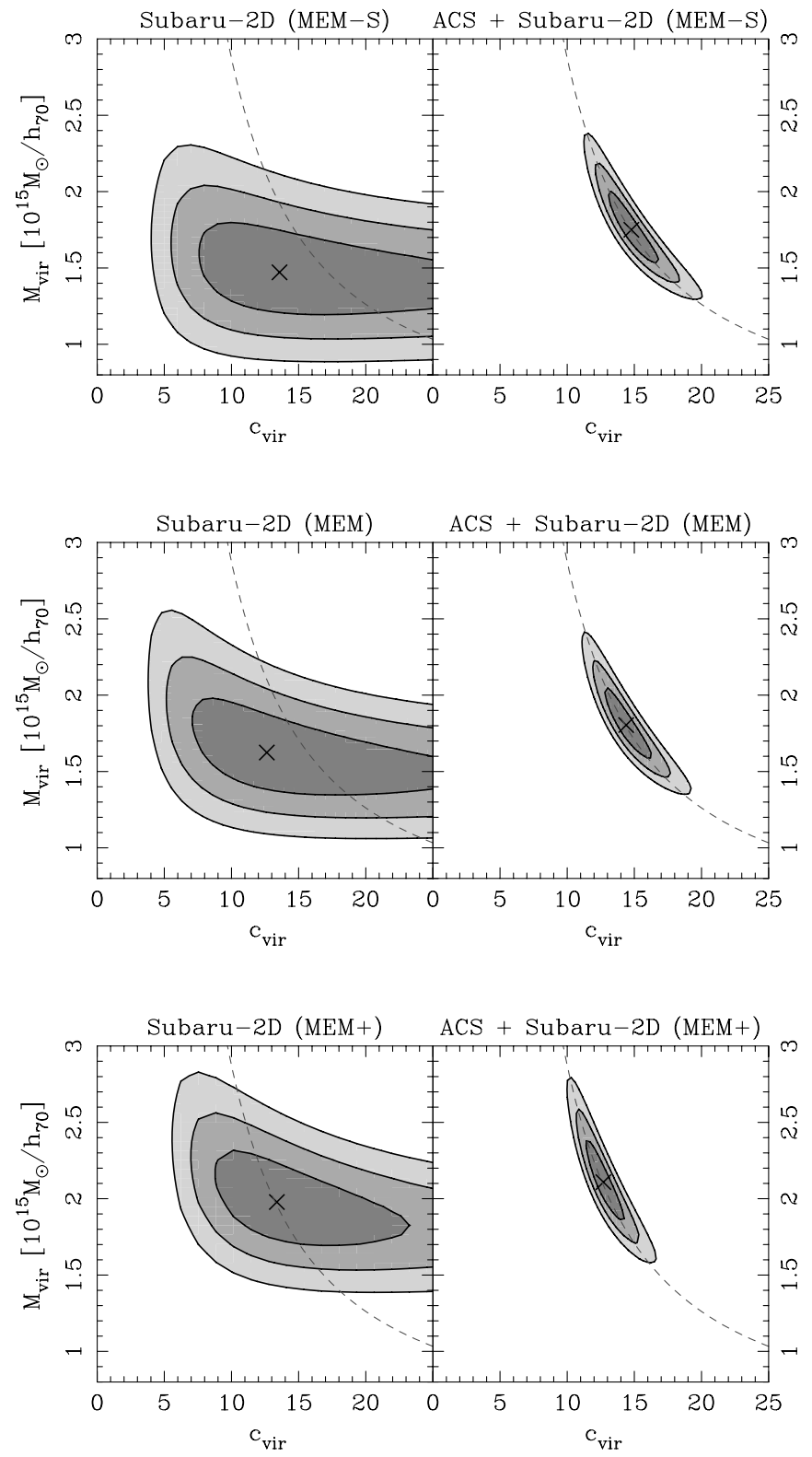

FIG. 14.- Joint constraints on the NFW model parameters, $\left(c_{\mathrm{vir}}, M_{\mathrm{vir}}\right)$, derived from gravitational lensing observations of A1689. Left: The $68 \%, 95 \%$, and $99.7 \%$ confidence levels $\left(\Delta \chi^{2}=2.3,6.17\right.$, and 11.8$)$ in the $\left(c_{\mathrm{vir}}, M_{\mathrm{vir}}\right)$-plane for the $2 \mathrm{D} \kappa$ map reconstructed from Subaru weak-lensing observations. Right: Same confidence levels as on the left, but for the joint ACS+Subaru-2D NFW fitting, incorporating the inner mass profile $\left(10 \mathrm{kpc} h^{-1} \lesssim r \lesssim 180 \mathrm{kpc} h^{-1}\right)$ constrained by ACS strong-lensing observations by B05b. The cross in each panel shows the best-fit set of the NFW model parameters. The top, middle, and bottom panels correspond to the results based on the 2D MEM+, MEM, and MEM-S reconstructions, respectively (see Table 2). The virial mass $M_{\mathrm{vir}}$ is well constrained by the Subaru data alone, while the Subaru constraint on the concentration $c_{\mathrm{vir}}$ is rather weak. The complementary ACS observations, when combined with the Subaru observations, significantly narrow down the uncertainties on $c_{\mathrm{vir}}$, placing stringent constraints on the inner mass profile. In each panel the observed constraint on the Einstein radius $\left(\theta_{\mathrm{E}} \simeq 45^{\prime \prime}\right.$ at $\left.z_{s}=1\right)$ is shown as a dashed curve. [See the electronic edition of the Journal for a color version of this figure.]

imply a shallower slope, as discussed in Medezinski et al. (2007) and Limousin et al. (2007).

It is useful to compare the results from different lensing studies in terms of the tangential distortion, $g_{+}$, that is directly observable in weak lensing. Figure 16 shows the radial profiles of $g_{+}$ and $g_{\times}$derived in B05a from the same red background sample as used in this work, where we have added the observed Einstein- radius constraint of $\theta_{\mathrm{E}}=45^{\prime \prime} \pm 5^{\prime \prime}\left(z_{s}=1\right)$, translated to the mean depth of the red background sample (§3.3), marking the point of maximum distortion, $g_{+}=1\left(\theta_{\mathrm{E}}=39^{\prime \prime} \pm 4^{\prime \prime}\right)$. The ACS+Subaru-2D NFW model (solid curve) fits reasonably well with the entire distortion profile, $r \sim[80,2000] \mathrm{kpc} h^{-1}$, although it slightly overpredicts the outer profile at $\theta \gtrsim 9^{\prime}$, meaning that the observed $g_{+}$profile is steeper at large radii, as pointed out in Medezinski et al. (2007). In contrast the best-fit NFW model of Limousin et al. (2007), shown with the dashed curve, is in agreement with the Subaru outer profile, particularly at $10^{\prime} \lesssim \theta \lesssim 18^{\prime}$, but underpredicts significantly the inner $g_{+}$profile, leading to a significant underprediction of the Einstein radius. On the other hand, the dotted curve in Figure 16 shows the NFW profile of Halkola et al. (2006), which is overall in good agreement with the observed $g_{+}$profile, and with the NFW prediction of this work, but increasingly overestimates the distortion signal with radius, and slightly underpredicts the Einstein radius. This is again consistent with that the discrepancy between the derived NFW parameters of this work and Halkola et al. (2006) is due to the relative weights in the fitting procedure assigned differently to the strong and weak-lensing measurements; that is, a relatively higher weight is given by Halkola et al. (2006) to the shallower inner profile at $r \lesssim 40 \mathrm{kpc} h^{-1}$ constrained by the radial arcs, ${ }^{10}$ and consequently, the location of the outer critical curve is slightly underpredicted. However, we argue this does not indicate a discrepancy between our strong and weak-lensing results, but is simply that the form of the NFW profile is not entirely consistent with our data ranging from 10 to $2000 \mathrm{kpc} h^{-1}$; the best-fit NFW profile is either too shallow at large radii or too steep at small radii, depending on the radial limits being examined.

\subsection{Systematic Errors of the Concentration Parameter}

In this subsection we address the issues of systematic errors on the halo concentration $c_{\text {vir }}$ inherent in our lensing measurements and analysis methods. Here we discuss the following potential sources of systematic uncertainty: (1) selection criteria for the red background sample (§3.3), (2) background redshift distribution (§ 3.3), (3) magnification analysis $(\S 4.2, \S 4.5)$, (4) inner boundary condition ( $\S 4.4)$, (5) strong-lensing model ( $(4.4$ and $\S 5.4 .2)$, (6) entropic prior (§ 4.3), and (7) shear calibration error. In Table 6 we summarize the systematic errors of the halo concentration, $c_{\mathrm{vir}}$, given in fraction of the best-fit value of $c_{\mathrm{vir}}=$ 12.7 obtained from the combined ACS and Subaru-2D (MEM+) data. Adding all potential sources of error in quadrature, the total uncertainty in our determination of the halo concentration is $c_{\mathrm{vir}}=12.7 \pm 1$ (statistical) \pm 2.8 (systematic).

\subsubsection{Background Selection Criteria}

As clearly demonstrated by B05a and Medezinski et al. (2007), a secure background selection is critical in the cluster weaklensing analysis, in order to avoid dilution of the distortion signal by contamination of unlensed cluster member galaxies. The degree of dilution, which is proportional to the fraction of cluster membership, is particularly prominent in the central region of rich clusters, as in the case of A1689 (see Medezinski et al. 2007). Practically, a reliable background sample can be defined by selecting galaxies with colors redder than the cluster E/S0 sequence (see $\S 3.3$ ). Figure 4 shows that the dilution effect becomes significant when the lower (bluer) color limit of the entire red sample is decreased below a color of $\Delta\left(V-i^{\prime}\right) \sim 0.1$, while no signature of systematic variations is seen when the lower

\footnotetext{
${ }^{10}$ The location of the observed radial critical curve is at $\theta \approx 17^{\prime \prime}$ as found in
} 
TABLE 5

Comparison between Best-Fitting NFW Parameters for A1689 from Different Observations and Methods

\begin{tabular}{|c|c|c|c|c|c|c|c|}
\hline Reference & Method $^{\text {a }}$ & $M_{\mathrm{vir}}{ }^{\mathrm{b}}$ & $c_{\mathrm{vir}}^{\mathrm{c}}$ & $M_{200}{ }^{\mathrm{d}}$ & $c_{200} \mathrm{e}$ & $\theta_{\mathrm{E}}^{\mathrm{f}}$ & Remarks \\
\hline King et al. (2002).. & WL & 1.0 & 6.1 & 0.84 & 4.8 & 11 & $\mathrm{ESO} / \mathrm{MPG}$ \\
\hline 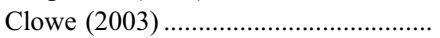 & WL & 1.3 & 9.9 & 1.1 & 7.9 & 22 & $\mathrm{ESO} / \mathrm{MPG}$ \\
\hline 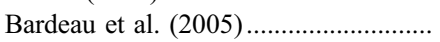 & WL & $1.72_{-0.6}^{+0.8}$ & $4.5_{-0.4}^{+0.6}$ & $1.41_{-0.47}^{+0.63}$ & $3.5_{-0.3}^{+0.5}$ & 3.5 & CFH12K \\
\hline B05b & $\mathrm{SL}$ & 3.7 & $8.2_{-1.8}^{+0.4}$ & 3.2 & $6.5_{-1.6}^{+1.3}$ & 44 & $\mathrm{ACS}$ \\
\hline В05а & $\mathrm{SL}+\mathrm{WL}$ & $1.93 \pm 0.20$ & $13.7_{-1.1}^{+1.8}$ & $1.72 \pm 0.19$ & $10.9_{-0.9}^{+1.0}$ & 45 & ACS + Subaru ( $\kappa$ profile $)$ \\
\hline 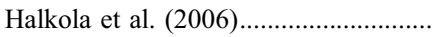 & SL & $3.55 \pm 0.4$ & $7.6 \pm 0.6$ & $3.05 \pm 0.3$ & $6.0 \pm 0.5$ & 37 & $\mathrm{ACS}$ \\
\hline Halkola et al. (2006).............................. & $\mathrm{SL}+\mathrm{WL}$ & $2.58 \pm 0.2$ & $9.6 \pm 0.6$ & $2.25 \pm 0.2$ & $7.6 \pm 0.5$ & 39 & ACS + Subaru ( $g_{+}$profile $)$ \\
\hline Bardeau et al. (2007) ............................. & WL & $2.35 \pm 0.4$ & $5.5 \pm 1.0$ & $1.97 \pm 0.3$ & $4.28 \pm 0.8$ & 12 & CFH12K \\
\hline Limousin et al. (2007) ............................ & WL & $1.51_{-0.2}^{+0.3}$ & $9.6 \pm 2.0$ & $1.32 \pm 0.2$ & $7.6 \pm 1.6$ & 24 & CFH12K \\
\hline 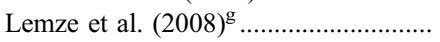 & $\mathrm{SL}+\mathrm{WL}+\mathrm{X}$ & $2.23 \pm 0.6^{\mathrm{h}}$ & $12.2_{-1}^{+0.9}$ & 1.98 & $9.7 \pm 0.8$ & 45 & ACS + Subaru + Chandra \\
\hline This work ..................... & WL & $1.97 \pm 0.20$ & $13.4_{-3.3}^{+5.4}$ & $1.76 \pm 0.20$ & $10.7_{-2.7}^{+4.5}$ & 45 & Subaru ( $\kappa$ map) \\
\hline 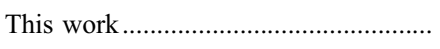 & $\mathrm{SL}+\mathrm{WL}$ & $2.10 \pm 0.17$ & $12.7_{-0.9}^{+1.0}$ & $1.86 \pm 0.16$ & $10.1_{-0.7}^{+0.8}$ & 45 & ACS + Subaru ( $\kappa$ map $)$ \\
\hline
\end{tabular}

NotE.-A similar table of best-fitting NFW parameters is found in Comerford \& Natarajan (2007; their Table 1) which also includes the results for other clusters as well as A1689.

a Analysis method.

b Virial mass $M_{\text {vir }}$ and $1 \sigma$ error in units of $10^{15} M_{\odot}$.

c Virial concentration $c_{\mathrm{vir}}=r_{\mathrm{vir}} / r_{s}$ and $1 \sigma$ error.

${ }^{\mathrm{d}} M_{200}$ and $1 \sigma$ error in units of $10^{15} M_{\odot}$.

e Specific concentration $c_{200}=r_{200} / r_{s}$ and $1 \sigma$ error.

${ }^{\mathrm{f}}$ Einstein radius in units of arcsec for a fiducial source at $z_{s}=1$, defined as $1=\bar{\kappa}\left(\theta_{\mathrm{E}}\right)$.

g Based on the Chandra X-ray data and the projected mass profile from the joint ACS and Subaru-1D analysis by B05a. Hydrostatic equilibrium assumed.

h Note that the outermost radius point of Lemze et al. (2008) is at $1.5 \mathrm{Mpc} h^{-1}$, which is smaller than the virial radius of A1689, $r_{\mathrm{vir}} \approx 2 \mathrm{Mpc} h^{-1}$. As compared to the

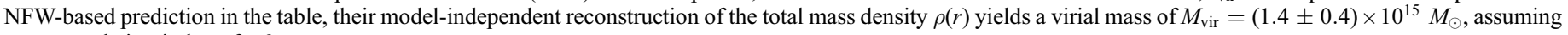
an extrapolation index of -3 .

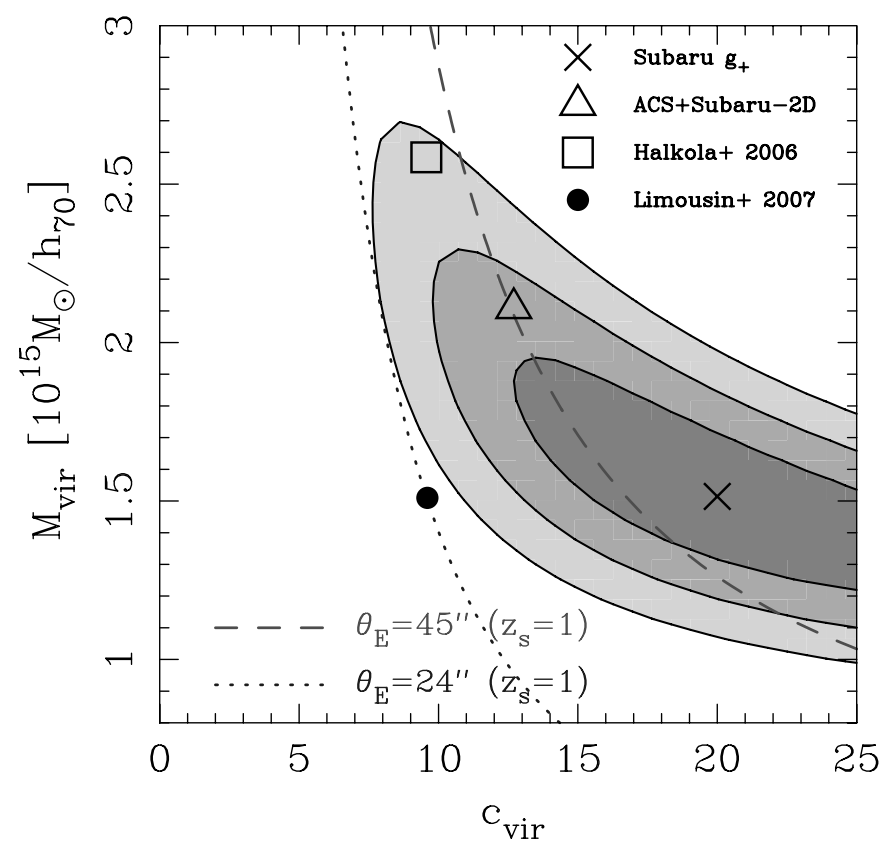

FIG. 15.- Joint constraints on the NFW model parameters, $\left(c_{\mathrm{vir}}, M_{\mathrm{vir}}\right)$ obtained from the Subaru tangential shear $\left(g_{+}\right)$profile of A1689 (see Fig. 1 of B05a). The cross shows the best-fitting set of the NFW parameters, and the contours show the $68 \%, 95 \%$, and $99.7 \%$ confidence levels $\left(\Delta \chi^{2}=2.3,6.17\right.$, and 11.8) in the $\left(c_{\mathrm{vir}}, M_{\mathrm{vir}}\right)$-plane. The observed constraint on the Einstein radius, $\theta_{\mathrm{E}} \simeq 45^{\prime \prime}$ at $z_{s}=1$, is shown as a dashed curve. The dotted curve shows the $c_{\mathrm{vir}}-M_{\mathrm{vir}}$ relation for $\theta_{\mathrm{E}}=24^{\prime \prime}$ at $z_{s}=1$. The triangle shows the best-fit set of $\left(c_{\mathrm{vir}}, M_{\mathrm{vir}}\right)$ for the combined ACS and Subaru-2D (MEM+) results. The square and circle show the best-fit sets of $\left(c_{\mathrm{vir}}, M_{\mathrm{vir}}\right)$ from the combined strong and weaklensing analysis of Halkola et al. (2006) and the weak-lensing analysis of Limousin et al. (2007), respectively. [See the electronic edition of the Journal for a color version of this figure.] color limit is increased above $\Delta\left(V-i^{\prime}\right) \sim 0.1$, ensuring that the dilution effect is almost negligible there. Based on this, we defined our red background sample by choosing a conservative color limit as $\Delta\left(V-i^{\prime}\right)>0.22$.

Here we vary the lower color limit of the red sample in the interval of $[0.1,0.5]$ where the dilution effect is negligible. At the lower color limit of $\sim 0.5$, however, the number of red galaxies is decreased by about $40 \%$ (see Fig. 4), when compared with our fiducial sample of $\Delta\left(V-i^{\prime}\right)>0.22$, leading to noisier results. We generate red samples at the lower color limit of $0.1,0.15$, $0.2, \ldots, 0.5$, and assess the scatter in the best-fit concentration parameter, $c_{\text {vir }}$. We find the error distribution is fairly random, with a small spread of $\Delta c_{\text {vir }} \sim \pm 0.5$, corresponding to a $\sim 4 \%$ fractional systematic error.

\subsubsection{Background Redshift Distribution}

Redshift information of background galaxies plays a crucial role in the determination of cluster mass profiles. In order to convert the observed lensing signal into physical mass units, one needs to evaluate the mean distance ratio $\left\langle D_{d s} / D_{s}\right\rangle$ over the redshift distribution of background galaxies $(\S 3.3)$. An overestimate of the source redshift will systematically lead to an underestimate of the cluster mass, and vice versa. In this way, the uncertainty in the background redshift distribution will lead to systematic errors of the cluster mass determination. The level of uncertainty depends on the lensing geometry and is less significant for low- $z$ clusters (say, $z_{d} \lesssim 0.2$ ). For purely weak-lensingbased data, this effect is less important for the determination of the halo concentration, because the identification of the inner characteristic radius $\left(r_{s}\right)$ is basically independent of the background redshift. However, when the weak-lensing measurements are combined with inner strong-lensing information, this depth information becomes crucial for the determination of halo concentration as well, because it determines the relative normalization between the inner and outer profiles. That is, an overestimate of the background redshift will cause an underestimate of the surface 


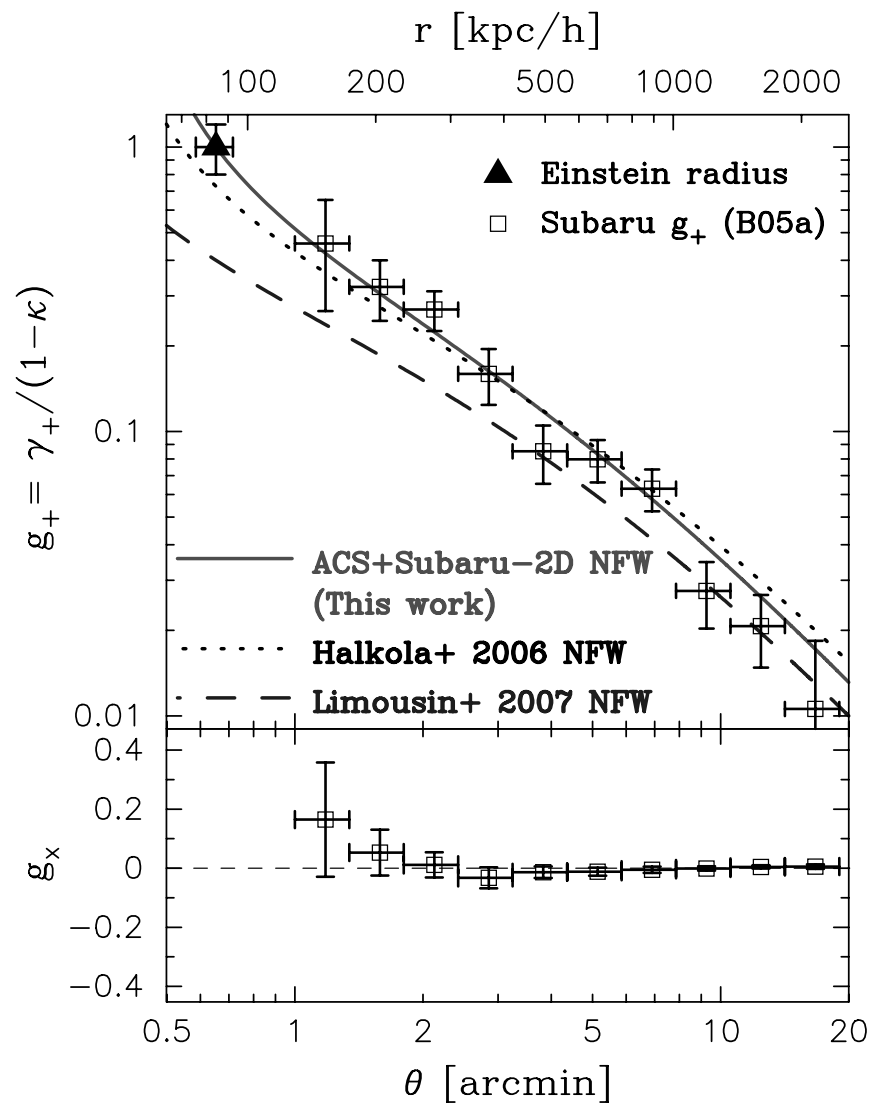

FIG. 16.-Top: Tangential distortion profile $g_{+}(\theta)$ (square) from the Subaru weak-lensing analysis of the red background sample (B05a). The solid curve shows the best-fit NFW profile derived from the joint strong and weak-lensing analysis of ACS and Subaru observations (this work), incorporating full distortion and magnification information. The Einstein radius constraint (triangle) of $\theta_{\mathrm{E}}=45^{\prime \prime}\left(z_{s}=1\right)$, determined from multiply lensed images in ACS observations (B05b), is translated to the corresponding depth of the Subaru red background sample using the ACS+Subaru-2D NFW model (solid curve), and added to the distortion profile $\left(g_{+}=1\right)$, marking the point of maximum distortion. The ACS + Subaru-2D NFW model (solid curve) fits well with the combined ACS and Subaru distortion information over the full range of data, $r=[80,2000] \mathrm{kpc} h^{-1}$, but somewhat overpredicts the outer distortion profile at $\theta \gtrsim 9^{\prime}\left(r \gtrsim 1.2 \mathrm{Mpc} h^{-1}\right)$. Also shown with the dashed curve is the best-fit NFW profile from the CFHT weaklensing analysis of Limousin et al. (2007), which, in contrast, is in good agreement with the Subaru outer profile at $\theta \gtrsim 4^{\prime}$, but significantly underpredicts the inner distortion profile and hence the Einstein radius. The dotted curve shows an NFW profile of Halkola et al. (2006) for a simultaneous fit to their ACS inner mass profile and the Subaru distortion profile of B05a shown here, but with a different weighting that prefers the inner strong-lensing based profile where the data imply a shallower slope (see Fig. 13). Bottom: Radial profile of the $45^{\circ}$ rotated component $g_{\times}(\theta)$ for the same Subaru red background sample (B05a). The $\times-$ component of the red galaxy sample is consistent with a null signal at all radii, indicating the reliability the Subaru distortion analysis. [See the electronic edition of the Journal for a color version of this figure.]

mass density, which will increase the difference between the inner profile derived from strong lensing and the outer profile from weak lensing, leading to an overprediction of the halo concentration.

Here we turn to assess the level of systematic error arising from the uncertainty in the background redshift distribution. Based on the multicolor photometry of Capak et al. (2004) in the HDF-N, we obtain a mean distance ratio of $\left\langle D_{d s} / D_{s}\right\rangle=0.693 \pm 0.02$, or a distance-equivalent redshift of $z_{s, D}=0.68 \pm 0.05$, for our color-magnitude-selected red background sample ( $\S 3.3$; see also Medezinski et al. 2007). A good agreement has been also found using the COSMOS deep multicolor photometric catalog of Capak et al. (2007), yielding a similar depth of $\left\langle D_{d s} / D_{s}\right\rangle \approx$ 0.703 (Broadhurst et al. 2008), suggesting that the field-to-field variance (cosmic variance) is not significant, and as small as the statistical uncertainty obtained here. We find this level of depth uncertainty will lead to only a $\sim 1 \%$ fractional systematic error of $c_{\text {vir }}$. Furthermore, we assign a conservative uncertainty in the (distance-equivalent) source redshift, $z_{s, D}=[0.7,1.0]$, and find a fractional systematic error of about $10 \%$ in the concentration parameter. It is interesting to note that B05a obtained the best-fit concentration parameter of $c_{\text {vir }}=13.7_{-1.1}^{+1.4}$ assuming a source redshift of $z_{s}\left(=z_{s, D}\right)=1$. This concentration is slightly higher $(\sim+8 \%)$ than the best-fit value of $c_{\text {vir }}=12.7 \pm 1$ found in this work, but this level of discrepancy can be easily reconciled by the systematic error in the assumed depth of B05a: applying this bias correction to the results of B05a yields $c_{\text {vir }} \approx 12.3$, which agrees quite well with the present results of $c_{\text {vir }}=12.7 \pm 1$.

Lensing magnification influences the observed surface density of background galaxies (Broadhurst et al. 1995), by expanding the observed solid angle of the background (area distortion), and decreasing the effective flux limit of the survey (flux amplification). Thus, the latter effect of magnification bias may change the redshift distribution of background galaxies depending on the distance from the cluster center, which could be a potential source of the systematic errors in the determination of the halo concentration. Our red background sample, however, is highly depleted (see Fig. 6), meaning that the area distortion effect is dominating over the flux amplification of fainter, distant background galaxies. Indeed, the unlensed count slope measured at our magnitude limit $i^{\prime}=25.5$ is $s=d \log N_{0}(<m) / d m \approx 0.22$, being fairly flat as compared to the blue sample with $s \approx 0.4$, close to the lensing invariant slope (see eq. [23]). Consequently, relatively few fainter objects are magnified into the sample even in the central cluster region, so that the magnification effect on the source redshift distribution is fairly negligible for the red background defined at fainter magnitude limits. For our sample, magnification at $\theta \sim 2^{\prime}$ is $\mu \simeq 1+2 \kappa \approx 1.2$, or about 0.2 mag of increased depth, corresponding to the fractional increase of only $\sim 5 \%$ in the number of red background galaxies. Furthermore, given the weak dependence of the redshift distribution of faint galaxies on apparent magnitude (e.g., Medezinski et al. 2007), we do not need to take this effect into serious consideration for our red background sample.

Still, it is instructive to consider the potential systematic bias caused by the magnification effect on the background redshift distribution. The net effect of depth correction is to reduce the central surface mass density of the lens, since we attribute the high lensing signal to the geometric information of the background. For purely weak-lensing-based data, this will lead to a lower concentration. However, when the strong-lensing information in the inner region is taken into account as well, this depth correction will further increase the difference between the inner and outer profiles derived from strong and weak lensing, respectively, thereby enhancing the concentration. However, the amount of correction is negligible in practice when the weak-lensing analysis is based on the red background galaxies as discussed above.

\subsubsection{Magnification Analysis}

Here we address the systematic uncertainties arising from a particular treatment and various cuts in the weak-lensing magnification analysis.

Mask area correction.-The masking effect due to cluster member galaxies and bright foreground objects acts to reduce the apparent number of background galaxies, and this reduction 
TABLE 6

Sources of Systematic Error and Their Effects on the Determination of the Halo Concentration Parameter $c_{\text {vir }}$

\begin{tabular}{lcc}
\hline \multicolumn{1}{c}{ Source of Error } & Uncertainty Range & $\begin{array}{c}\text { Fractional Error in } c_{\mathrm{vir}} \\
(\%)\end{array}$ \\
\hline Lower color limit for the red sample $\ldots \ldots \ldots \ldots \ldots \ldots \ldots \ldots \ldots$ \\
Source redshift
\end{tabular}

Note.-The systematic errors are presented in fraction of $c_{\text {vir }}=12.7$ derived from a joint fit to the ACS-based inner $\kappa$ profile of B05b and the Subaru-based $\kappa$ map reconstructed with the MEM+ method.

${ }^{\text {a }}$ Effective source redshift $z_{s, D}$ equivalent to the mean distance ratio $\left\langle D_{d s} / D_{s}\right\rangle$ defined by eq. (18).

b ${ }_{s}=d \log N(<m) / d m$.

c Einstein-radius constraint $\left(\theta_{\mathrm{E}}=45^{\prime \prime}\right.$ for $\left.z_{s}=1\right)$ used instead of the ACS inner mass profile of B05b.

increases toward the cluster center, leading to an overestimate of the central depletion signal without the masking correction. In the present study the mask area of bright objects is evaluated as the area inside the ellipse of $\nu_{\text {mask }}$ times the major (A_IMAGE) and minor (B_IMAGE) axes computed from SExtractor (see Cobb et al. 2006 for a similar discussion), where the multiplier is chosen as $\nu_{\text {mask }}=3$ so that the ellipse is visually consistent with the isophotal detection limit in our SExtractor configuration $(\S 3.1)$. Here we adopt a conservative uncertainty of $\nu_{\text {mask }}=3 \pm 1$ on the masking factor, and find the corresponding systematic uncertainty of $\pm 7 \%$ in the halo concentration. The lower limit on $c_{\mathrm{vir}}$ is obtained for $\nu_{\text {mask }}=2$, when the mask area correction is underestimated and hence the depletion signal is overestimated.

Clustering rejection.- Similarly, we adopt a conservative uncertainty of $\nu_{\text {clust }}=4 \pm 1$ in the rejection threshold $\nu_{\text {clust }}$, which has been introduced to downweight locally the intrinsic clustering noise which otherwise perturbs the depletion signal ( $§ 4.5$ ). This yields a fractional uncertainty of only $\pm 1.5 \%$ in $c_{\text {vir }}$. We note that the intrinsic clustering of background galaxies is a local effect, and hence it does not affect significantly the radial profile fitting.

Unlensed count slope.-The conversion from the observed counts of the red background sample into the magnification bias $\delta_{\mu}$ (eq. [23]) depends on the slope parameter $s=d \log N_{0}(<m) / d m$ of the unlensed number counts ( $\S 4.2)$, which was estimated as $s=0.22 \pm 0.03$ from the outer region $\gtrsim 10^{\prime}$ where the magnification effect is negligibly small $\left(\kappa, \delta_{\mu} \lesssim 0.01\right)$. We find that this level of uncertainty in $s$ will cause an uncertainty in $c_{\text {vir }}$ of about $3.5 \%$.

\subsubsection{Inner Boundary Condition}

Any mass reconstruction technique based on the gravitational shear field involves a nonlocal process (see eq. [6]), and hence it is crucial to have a proper boundary condition for an accurate determination of the cluster mass profile. In particular, our MEM+ method is based on the ACS strong-lensing information, which is incorporated as an inner boundary condition on the central pixel, $\kappa_{c}(\S 4.4)$. B05a showed that the combined ACS and Subaru mass profile can be well fitted by a high-concentration NFW profile $\left(c_{\text {vir }} \sim 14\right)$ over the full range of ACS and Subaru data, $r=\left[10^{-2}, 2\right] \mathrm{Mpc} h^{-1}$. However, it is also found in B05a that this high-concentration model somewhat overestimates the inner slope at $r \lesssim 40 \mathrm{kpc} h^{-1}$ (see Fig. 13). This model yields a central surface mass density of $\kappa_{c}\left(z_{s}=1\right) \approx 0.810$, which is slightly higher than, but still consistent with, the prediction based on
$\mathrm{B} 05 \mathrm{~b}$ adopted in the present work, $\kappa_{c}\left(z_{s}=1\right)=0.781 \pm 0.1$, within the $1 \sigma$ statistical uncertainty. The mass profile of A1689 has also been examined by Limousin et al. (2007) using independent weak-lensing shape measurements from CFHT12K data. Their best-fit NFW model, however, underpredicts the observed Einstein radius $(\S 5.4 .3)$ and accordingly yields a much lower central value of $\kappa_{c}\left(z_{s}=1\right) \approx 0.527$, which we take as the lower limit on $\kappa_{c}\left(z_{s}=1\right)$. Allowing for a conservative uncertainty of $\kappa_{c}\left(z_{s}=1\right)=0.78_{-0.25}^{+0.1}$, corresponding to $\kappa_{c}\left(z_{s, D}=0.68\right)=$ $0.70_{-0.22}^{+0.09}$ for the effective depth of our red sample, we find a $\pm 10 \%$ fractional systematic uncertainty in the halo concentration, $c_{\text {vir. }}$.

\subsubsection{Inner Strong-Lensing Information}

The inner strong-lensing information provides strong constraints on the halo concentration parameter as demonstrated in Figure 14. In the present work, the ACS-derived inner mass profile of B05b is specifically used to determine the NFW halo parameters of A1689 in the strongly lensed region, $r=[10,130] \mathrm{kpc} h^{-1}$. It is practically difficult to assess potential systematic errors introduced in strong-lensing modeling because of the complex, nonlinear error propagation (B05b). Instead, here we simply estimate the level of uncertainty by a comparison with the result obtained with the model-independent constraint on the Einstein radius $\theta_{\mathrm{E}}$ (see $\S 5.4 .2$ ) based on multiply lensed images identified in the ACS observations of B05b. Both the strong-lensing analysis of B05b and Limousin et al. (2007) yield a consistent value for the projected mass interior to $45^{\prime \prime}$ of $M_{2 \mathrm{D}}\left(45^{\prime \prime}\right) \approx 2 \times 10^{14} M_{\odot}$, or equivalently, $\bar{\kappa}\left(45^{\prime \prime}\right)=1$ for a source redshift of $z_{s}=1$, i.e., $\theta_{\mathrm{E}}=45^{\prime \prime}$ at $z_{s}=1$. In contrast to the fit to the inner mass profile, this Einstein-radius information provides an integrated constraint on the inner mass profile interior to $45^{\prime \prime}$, or $r \approx 100 \mathrm{kpc} h^{-1}$ in projected radius. We find that a joint fit of the Subaru $\kappa$ map and the Einstein-radius constraint yields a slightly higher concentration of $c_{\text {vir }}=14.0_{-2.1}^{+2.5}$ (see Table 4), corresponding to a fractional increase of about $10 \%$. This tendency is also found for the results with the shear-based $1 \mathrm{D}$ mass reconstruction from the $\zeta_{c^{-}}$ statistic measurements (see Table 4).

\subsubsection{Entropic Prior}

A particular choice of the regularization could be a potential source of the systematic errors in the cluster mass reconstruction. In our mass reconstructions the model parameter $m$ of the entropy prior is fixed, but the Bayesian value of the regularization parameter $\alpha$ that satisfies equation (31) is obtained for a given 
value of $m$. When we vary the value of $m$ over a relevant range of the cluster lensing signal, $m=[0.1,0.9](m=0.5 \pm 0.4)$, we find the error distribution in the resulting value of $c_{\mathrm{vir}}$ is almost random with a small spread of $\pm 10 \%$.

Furthermore, a particular choice of the entropy function may lead to some systematic bias in the determination of cluster mass profiles. To check this possibility, here we simply compare the present results from the MEM+ reconstruction with earlier 1D maximum likelihood results of B05a, both of which are based on the same distortion and magnification data, and adopt the ACSbased inner boundary conditions. After the correction for the systematic bias ( $\S 5.5 .1)$ the best-fit concentration of B05a is $c_{\mathrm{vir}} \approx 12.3$, which is in good agreement with $c_{\mathrm{vir}}=12.7 \pm 1$ obtained with the entropy regularization. Thus, it is likely that the level of systematic uncertainty due to the particular choice of the entropy regularization is negligibly small as compared to other sources of the systematic errors.

Our use of the entropy prior in the low $\mathrm{S} / \mathrm{N}$ regime might potentially induce some slight bias in the regularized maximum likelihood solution, and/or some slight non-Gaussianity in the error distribution, underestimating the actual error bars for the mass reconstruction and the NFW halo parameters. However, our results show good consistency between the entropy-regularized reconstructions and other standard reconstructions within the statistical uncertainties. Thus, it seems this bias is not significant for this work.

\subsubsection{Shear Calibration Error}

A shear calibration error is one of the systematic errors that could bias the weak-lensing shape measurements (Heymans et al. 2006; Massey et al. 2007) and potentially have some influence on recovered mass profiles. To assess this possibility we have measured the strength of the weak-lensing signal as a function of magnitude limit for our background galaxy sample. We found no particular tendency toward a loss of the weak-lensing signal with apparent magnitude for red background galaxies, which span over a wide range of sizes. This is comforting and consistent with the expectations of the model-independent KSB95+ based technique for which the recovered signal should match reality within the noise. We note that at the very weak-lensing limit, our distortion measurements are quite consistent with an independent estimate of the weak-lensing signal by Limousin et al. (2007).

However, we have found that for blue background galaxies there is a significant loss of the signal at faint magnitudes, and this raises the worrying question of unresolvable $\mathrm{H}$ II regions which we know do become prevalent at faint blue magnitudes, acting effectively as point sources and hence reducing the weaklensing signal. Such objects are not included in our analysis, so as not to bias our lensing measurements. The STEP project, aimed at assessing signal which may be lost in ground based data, described in Heymans et al. (2006) and Massey et al. (2007), does not allow for unresolvable sources within galaxies, as it is inherently assumed that galaxies are continuously resolvable, so that stretched HST ACS images of faint galaxies are taken to be perfectly empirical representations of reality for the purpose of calibrating galaxies dominated by bright $\mathrm{H}$ II regions.

Furthermore, we have found a good consistency between the purely shear-based results (e.g., $\zeta$-statistic based $1 \mathrm{D}$ reconstruction) and the results based on the combined distortion and magnification data (e.g., MEM+ results, B05a results), implying that any shear calibration error is not noticeable at the level of our analysis, otherwise we would see inconsistency with our magnification analysis.

\section{SUMMARY AND DISCUSSION}

In this paper, we have derived a projected 2D mass map of the well-studied lensing cluster A1689 $(z=0.183)$ based on an entropy-regularized maximum likelihood combination of the lens magnification and distortion of red background galaxies registered in deep Subaru images. The combination of distortion and magnification data breaks the mass-sheet degeneracy inherent in all reconstruction methods based on distortion information alone. The method is not restricted to the weak-lensing regime but applies to the whole area outside the tangential critical curve, where nonlinearity between the surface mass-density and the observables extends to a radius of a few arcminutes. The strong-lensing information from ACS observations was also readily incorporated in this maximum likelihood approach $(\S 4.4)$. We also utilized the distortion measurements to locally downweight the intrinsic clustering noise in the magnification measurements, which otherwise perturbs the depletion signal (§ 4.5). The resulting 2D map showed that the projected surface density of A1689 is smoothly varying and symmetric, similar to the distribution of cluster members. The 2D mass map is well fitted by the Navarro-Frenk-White model, with a continuously steepening profile, but the concentration parameter much higher than expected for its virial mass $\left(\sim 2 \times 10^{15} M_{\odot}\right)$, according to the clear predictions of standard $\Lambda$ CDM (Bullock et al. 2001; Neto et al. 2007). For consistency we have compared the best-fitting NFW parameters obtained from different combinations of data sets, boundary conditions, and weak-lensing techniques ( $(5.4 .3)$. We find that all of the reconstructions tested here are consistent with a virial mass in the range, $M_{\mathrm{vir}}=(1.5-2.1) \times 10^{15} M_{\odot}$, and the combined ACS and Subaru-2D mass reconstruction yields a tight constraint on the concentration parameter, $c_{\mathrm{vir}}=12.7_{-0.9}^{+1.0}$ $\left(c_{200} \sim 10\right)$, improving on the statistical accuracy of our earlier $1 \mathrm{D}$ analysis (B05a). Very good agreement is found between the present full 2D reconstruction and the earlier 1D reconstruction (B05a), both of which are based on the same distortion and magnification measurements, supporting the assumption of quasicircular symmetry in the projected mass distribution. We have also explored potential sources of systematic error on the concentration parameter, such as the uncertainties in background redshift distribution, selection criteria for the red background sample, and strong-lensing modeling. Taking into account all of the systematic errors as well as the statistical uncertainty, our constraint on the concentration is $c_{\text {vir }}=12.7 \pm 1$ (stat.) \pm 2.8 (systematic).

For clusters well fitted by an NFW profile, the derived virial mass of a cluster and the concentration parameter can be used to find the Einstein radius $\theta_{\mathrm{E}}$, through the simple relationship $1=$ $\bar{\kappa}_{\mathrm{NFW}}\left(\theta_{\mathrm{E}}\right)$ (see Appendix B). For A1689 with $M_{\mathrm{vir}}=(2.1 \pm$ $0.2) \times 10^{15} M_{\odot}$ and $c_{\text {vir }}=12.7_{-0.9}^{+1.0}$ (only statistical errors quoted), this yields an Einstein radius of $45^{\prime \prime} \pm 6^{\prime \prime}$ at $z_{s}=1$, or $53^{\prime \prime} \pm 7^{\prime \prime}$ at $z_{s}=3$, in very good agreement with the mean estimated radius of $\sim 50^{\prime \prime}$, based on the locations of the multiple images (Table 2 of B05b). The strong-lensing mass model of Limousin et al. (2007), based on the multiple images identified by B05b, properly reproduces the observed Einstein radius of $45^{\prime \prime}$ at $z_{s}=1$, consistent with the strong-lensing mass model of B05b (see $\S$ 5.5.5). In contrast, an Einstein radius of $24^{\prime \prime} \pm 11^{\prime \prime}$ at $z_{s}=1$ is implied by the NFW fit to this cluster by Limousin et al. (2007), to independent weak-lensing data from CFHT ( $c_{\text {vir }} \sim 9.6, M_{\text {vir }} \sim$ $1.5 \times 10^{15} M_{\odot}$; see Table 5, Figs. 15 and 16). This discrepancy may be attributed to a degree of contamination of the sample of galaxies used to define the lensed background, which, as pointed out in B05a, can drag down the weak-lensing signal if accidentally included in the background sample, and preferentially 
so at small radius (see Fig. 16) where the ratio of cluster members compared with background is much higher, resulting in a shallow $g_{+}$profile and hence a lower concentration fit.

Taking into account the systematic errors ( $\S 5.5)$, combined ACS and Subaru constraints allow a shallower (but still steeper than theoretically expected) mass profile with $c_{\text {vir }}=9-10$, similar to the values found in Halkola et al. (2006) and Limousin et al. (2007). This, however, does not simply mean that the discrepancy between different lensing studies is fully solved: when the NFW model is normalized to reproduce the observed Einstein radius $\left(45^{\prime \prime}\right.$ at $\left.z_{s}=1\right)$, then this concentration would indicate a large virial mass of $M_{\mathrm{vir}}=(3-3.3) \times 10^{15} M_{\odot}$ (see Figs. 14 and 15), which however is considerably higher than the mass estimates derived from the X-ray observations $\left(M_{\mathrm{vir}} \approx 10^{15} M_{\odot}\right.$ in Andersson $\&$ Madejski $2004 ; M_{\text {vir }} \approx 1.4 \times 10^{15} M_{\odot}$ in Lemze et al. 2008). The discrepancy between the present results and the results by Halkola et al. (2006) can be explained by the relative weights in the least $\chi^{2}$-fitting, assigned differently to the ACSand Subaru-based measurements, indicating slight deviation of the observed profile from the NFW predictions (see discussion in $\S$ 5.4.3; also see discussion in Medezinski et al. 2007). This is seen at the innermost radii $r \lesssim 40 \mathrm{kpc} h^{-1}$ (Fig. 13), where the ACS data indicate a shallower profile. Consequently, when one prefers the innermost region to fit the data, this could lead to a lower concentration $\left(c_{\mathrm{vir}}=9-10\right)$ as favored by the shallower slope in the innermost region, and to a lower value for the Einstein radius when the data at outer critical radius are less weighted. Nonetheless, our best-fitting NFW model provides a good approximation to our data over the radii we have considered, $r=\left[10^{-2}, 2\right] \mathrm{Mpc} h^{-1}$.

B05a demonstrate that dilution of the lensing signal is certainly the cause of the very low concentration fit $\left(c_{\mathrm{vir}} \sim 4.5\right.$, Table 5) obtained by Bardeau et al. (2005), due to the inclusion of relatively blue cluster members in the definition of the background sample of the same CFHT weak-lensing data as Limousin et al. (2007), and for which the equivalent Einstein radius is only $\sim 4^{\prime \prime}$ (at $z_{s}=1$, see Table 5). On the other hand, the dilution of the lensing signal caused by cluster members can be used to derive the proportion of galaxies statistically belonging to the cluster by comparing the undiluted red background distortion signal with the radial distortion profile of color-magnitude space occupied by the cluster members, but including inevitable background galaxies falling in the same space (Medezinski et al. 2007). This technique allows the light profile of the cluster to be determined in a way which is independent of the number density fluctuations in the background population, which otherwise limit the calculation of the cluster light profiles and luminosity functions from counts of member galaxies. The resulting light profile can be compared with the mass profile to examine the radial behavior of $M / L$ (Medezinski et al. 2007).

A recent joint X-ray and lensing analysis of A1689 by Lemze et al. (2008) also produces very similar concentration, $c_{\mathrm{vir}}=$ $12.2_{-1}^{+0.9}$, and virial mass, $M_{\text {vir }} \sim(1.4 \pm 0.4) \times 10^{15} M_{\odot}$, to that derived here in our analysis (Table 5). This is derived from a model-independent approach to the X-ray emission profile and the projected lensing mass profile, assuming hydrostatic equilibrium, utilizing the mass profile derived in B05a. Interestingly, the observed temperature profile falls short of the predicted temperature profile derived from the joint fit, and this may imply that the gas distribution is clumpy on small scales, in the form of a higher density cold gas phase (Lemze et al. 2008). Moreover, this anomaly seems to be consistent with conclusions regarding gas substructure in the recent detailed hydrodynamical simulations of cluster gas by Kawahara et al. (2007), implying that other similar detailed lensing-X-ray studies should also show a similar temperature discrepancy.

Great progress continues to be made in the detailed predictions of $\Lambda C D M$, particularly on cluster scales where gas cooling is not a worry. Recently the whole Millennium survey (Springel et al. 2005) has been converted into the observer's frame following the full geodesics through the volume to simulate the effect of structure on the light received by an observer (Hilbert et al. 2007). This work has shown that although in general clusters form in overdense regions, the material associated with a given cluster in the form of extended groups and filaments outside the virial radius of the cluster is of relatively low mass contrast compared to the projected mass due to the cluster itself, and therefore lensingbased projected masses of clusters are not overestimated by more that a few percent (Hilbert et al. 2007).

This simulation has also been used to better define the relationship between the concentration parameter and the virial mass of halos, over the full range of mass from galaxies up to the most massive cluster-sized halos, in the context of standard $\Lambda \mathrm{CDM}$ (Neto et al. 2007). A clear prediction has emerged that most massive halos generated in these simulations have the lower concentration $\left(c_{\mathrm{vir}} \sim 5\right)$, and the cause of this is in part attributed to the generally later collapse of the more massive halos reflecting the lower mean density of the universe. For example, at $z_{\mathrm{vir}}=$ 0.183 , for the standard choice of cosmological parameters the criterion for virialization is $\bar{\rho}\left(<r_{\text {vir }}\right) \sim 115 \rho_{\text {crit }}\left(z_{\text {vir }}\right) \sim 277 \bar{\rho}\left(z_{\text {vir }}\right)$. One possibility to achieve earlier formation of massive clusters is to allow deviation from Gaussianity of the primordial density fluctuations in the early universe (e.g., Grossi et al. 2007; Sadeh et al. 2007).

A degree of triaxiality is inevitable for collisionless gravitationally collapsed structures. Discussion of the likely effect of triaxiality on the measurements of lensing properties has been examined analytically (Oguri et al. 2005; Sereno 2007; Corless \& King 2007) and in numerical investigations (Jing \& Suto 2002; Hennawi et al. 2007). A bias in favor of prolate structure pointed to the observer is unavoidable at some level, as this orientation boosts the projected surface mass density and hence the lensing signal. This effect has been evaluated in the context of the CDM model and serves as a guide to the likely degree of bias which may affect lensing work. Hennawi et al. (2007) conducted a detailed study of the properties of lensing cluster population identified in $\Lambda$ CDM cosmological $N$-body simulations. The level of bias in terms of the concentration parameter derived from 2D lensing measurements was explicitly estimated and found to amount to an $\sim 34 \%$ increase, which results from a combination of two effects, namely, the orientation bias $(\sim 19 \%)$ due to halo triaxiality and the selection effect toward higher $3 \mathrm{D}$ concentrations $(\sim 18 \%)$. The level of correction for the orientation bias is also derived from semianalytical representation of simulated CDM triaxial halos by Oguri et al. (2005). The anomalously high concentrations of $c_{\text {vir }} \gtrsim 13$, such as found for A1689, CL 0024+ 1654 (Kneib et al. 2003), and MS 2137-23 (Gavazzi et al. 2003), appear inconsistent with the distribution of concentrations found in detailed simulations of Hennawi et al. (2007), which predict that only $<2 \%$ of lensing clusters should have such high concentrations. It is also unlikely that the baryonic component in these massive clusters increases the concentrations over the $\Lambda$ CDM prediction for dark matter halos (see discussion in Hennawi et al. 2007, B05b, and Broadhurst \& Barkana 2008).

A chance projection of foreground/background structure along the line of sight can potentially influences projected lensing observations, boosting the surface mass density locally and hence the concentration. The ACS strong-lensing analysis of B05b 
revealed the secondary mass clump in the central region of A1689 associated with a small clump of galaxies. The existence of this subclump has been suggested by earlier observations, such as the spectroscopic study of Teague et al. (1990) and Czoske (2004), and the X-ray study of Andersson \& Madejski (2004). No obvious substructure is visible in a large spectroscopic sample of 525 cluster members identified in Czoske (2004), in contrast to earlier work of Teague et al. (1990) based on a smaller sample of 176 identified cluster members. Recently, this secondary mass clump has also been directly detected by the weak-lensing flexion analyses by Leonard et al. (2007) and Okura et al. (2008) based on the ACS and Subaru data, respectively. However, the detailed ACS strong-lensing analyses based on multiply imaged background galaxies showed that the mass contribution of the secondary mass clump is only a small fraction of the main cluster component (Figs. 21 and 22 of B05b), implying a lower $M / L$ for the subclump than for the main cluster, which is tightly constrained by the geometrical positions of sets of multiply lensed images, or the location of critical curves.

In the near future, the question of the effect of triaxiality on lensing-based cluster mass profiles may be examined empirically. For example, the total X-ray luminosity or the total lensing based mass of a cluster should not depend on the orientation with respect to the line of sight, whereas the concentration parameter and the Einstein radius will be affected, and hence expanded lensing studies could in principle reveal whether relaxed clusters of fixed mass or fixed X-ray luminosity tend to have consistent lensing based concentrations, or instead a broader distribution may be uncovered, with a mean concentration smaller than derived for A1689, indicating triaxiality produces a significant bias. Current indications based on several massive clusters favor the NFW profile, but with consistent concentrations (Broadhurst et al. 2008), similar in value to A1689, underscoring the tension between detailed lensing-based mass profiles and the predictions of standard $\Lambda \mathrm{CDM}$.

We thank the anonymous referee for a careful reading of the manuscript and for providing invaluable comments. We are grateful to Masahiro Takada for valuable discussions and comments. We thank J.-H. Proty Wu, Elinor Medezinski, Guo-Chin Liu, Ue-Li Pen, Masamune Oguri, and Sandor Molnar for fruitful discussions. We thank Nick Kaiser for making the IMCAT package publicly available. K. U. thanks Ludovic Van Waerbeke for kindly providing his code for a maximum likelihood mass reconstruction. Part of this work is based on data collected at the Subaru Telescope, which is operated by the National Astronomical Society of Japan. This work in part supported by the National Science Council of Taiwan under the grant NSC952112-M-001-074-MY2.

\section{APPENDIX A}

\section{DISCRETIZED ESTIMATOR FOR THE LENSING CONVERGENCE}

In this Appendix, we aim to derive an expression for the discrete convergence profile using the weak-lensing aperture densitometry $\zeta_{c}(\theta)$ given by equation (46). In the continuous limit, the averaged convergence $\bar{\kappa}(\theta)$ and the convergence $\kappa(\theta)$ are related by

$$
\begin{aligned}
& \bar{\kappa}(\theta)=\frac{2}{\theta^{2}} \int_{0}^{\theta} d \ln \theta^{\prime} \theta^{\prime 2} \kappa\left(\theta^{\prime}\right), \\
& \kappa(\theta)=\frac{1}{2 \theta^{2}} \frac{d\left(\theta^{2} \bar{\kappa}\right)}{d \ln \theta}
\end{aligned}
$$

For a given set of annular radii $\theta_{m}(m=1,2, \ldots, N)$, discretized estimators can be written in the following way:

$$
\begin{aligned}
& \bar{\kappa}_{m} \equiv \bar{\kappa}\left(\theta_{m}\right)=\frac{2}{\theta_{m}^{2}} \sum_{l=1}^{m-1} \Delta \ln \theta_{l} \bar{\theta}_{l}^{2} \kappa\left(\bar{\theta}_{l}\right), \\
& \kappa_{l} \equiv \kappa\left(\bar{\theta}_{l}\right)=\alpha_{2}^{l} \bar{\kappa}_{l+1}-\alpha_{1}^{l} \bar{\kappa}_{l}, \quad(l=1,2, \ldots, N-1),
\end{aligned}
$$

where

$$
\alpha_{1}^{l}=\frac{1}{2 \Delta \ln \theta_{l}}\left(\frac{\theta_{l}}{\bar{\theta}_{l}}\right)^{2}, \quad \alpha_{2}^{l}=\frac{1}{2 \Delta \ln \theta_{l}}\left(\frac{\theta_{l+1}}{\bar{\theta}_{l}}\right)^{2}
$$

with $\Delta \ln \theta_{l} \equiv\left(\theta_{l+1}-\theta_{l}\right) / \bar{\theta}_{l}$ and $\bar{\theta}_{l}$ being the area-weighted center of the $l$ th annulus defined by $\theta_{l}$ and $\theta_{l+1}$; in the continuous limit, we have

$$
\begin{aligned}
\bar{\theta}_{l} & \equiv 2 \int_{\theta_{l}}^{\theta_{l+1}} d \theta^{\prime} \theta^{2} /\left(\theta_{l+1}^{2}-\theta_{l}^{2}\right) \\
& =\frac{2}{3} \frac{\theta_{l}^{2}+\theta_{l+1}^{2}+\theta_{l} \theta_{l+1}}{\theta_{l}+\theta_{l+1}}
\end{aligned}
$$


The technique of the aperture densitometry (Fahlman et al. 1994; Clowe et al. 2000) allows us to measure the azimuthally averaged convergence $\bar{\kappa}(\theta)$ up to an additive constant $\bar{\kappa}_{b}$, corresponding to the mean convergence in the outer background annulus with inner and outer radii of $\theta_{\text {inn }}$ and $\theta_{\text {out }}$, respectively $(\S 5.3)$ :

$$
\bar{\kappa}(\theta)=\zeta_{c}(\theta)+\bar{\kappa}_{b}
$$

Substituting equation (A7) into equation (A4) yields the desired expression as

$$
\kappa\left(\theta_{l}\right)=\alpha_{2}^{l} \zeta_{c}\left(\theta_{l+1}\right)-\alpha_{1}^{l} \zeta_{c}\left(\theta_{l}\right)+\left(\alpha_{2}^{l}-\alpha_{1}^{l}\right) \bar{\kappa}_{b} .
$$

\section{APPENDIX B}

\section{THE NFW LENS MODEL}

The NFW universal density profile has a two-parameter functional form as

$$
\rho_{\mathrm{NFW}}(r)=\frac{\rho_{s}}{\left(r / r_{s}\right)\left(1+r / r_{s}\right)^{2}},
$$

where $\rho_{s}$ is a characteristic inner density, and $r_{s}$ is a characteristic inner radius. The virial properties are related. Instead of using $\rho_{s}$ and $r_{s}$, we introduce for an NFW halo the virial mass $M_{\mathrm{vir}}$ and the concentration parameter, $c_{\mathrm{vir}} \equiv r_{\mathrm{vir}} / r_{s}$, defined as the ratio of the virial radius $r_{\text {vir }}$ to the scale radius. The virial mass and virial radius are related through the following equation:

$$
M_{\mathrm{vir}}=\frac{4 \pi}{3} \bar{\rho}\left(z_{\mathrm{vir}}\right) \Delta_{\mathrm{vir}} r_{\mathrm{vir}}^{3}
$$

where $\Delta_{\text {vir }}$ is the mean overdensity with respect to the mean cosmic density $\bar{\rho}\left(z_{\mathrm{vir}}\right)$ at the virialization epoch $z_{\mathrm{vir}}$, predicted by the dissipationless spherical top-hat collapse model (Peebles 1980; Eke et al. 1996). We assume the cluster redshift $z_{d}$ is equal to the cluster virial redshift $z_{\text {vir. }}$. We use the following fitting formula in a flat three-space with cosmological constant (see Oguri et al. 2001):

$$
\Delta_{\mathrm{vir}}=18 \pi^{2}\left(1+0.4093 \omega_{\mathrm{vir}}^{0.9052}\right)
$$

where $\omega_{\text {vir }} \equiv 1 / \Omega_{m}\left(z_{\text {vir }}\right)-1$.

The inner density $\rho_{s}$ can be then expressed in terms of other virial properties of the NFW halo:

$$
\rho_{s}=\bar{\rho}\left(z_{\mathrm{vir}}\right) \frac{\Delta_{\mathrm{vir}}}{3} \frac{c_{\mathrm{vir}}^{3}}{\ln \left(1+c_{\mathrm{vir}}\right)-c_{\mathrm{vir}} /\left(1+c_{\mathrm{vir}}\right)} .
$$

Hence, for a given cosmological model and a halo virial redshift, we can specify the NFW model with the halo virial mass $M_{\text {vir }}$ and the halo concentration parameter $c_{\text {vir }}$.

For an NFW profile, it is useful to decompose the convergence $\kappa(\theta)$ and the averaged convergence $\bar{\kappa}(\theta)$ as

$$
\begin{aligned}
& \kappa_{\mathrm{NFW}}(x)=\frac{b}{2} f(x), \\
& \bar{\kappa}_{\mathrm{NFW}}(x)=\frac{b}{x^{2}} g(x),
\end{aligned}
$$

where $b=4 \rho_{s} r_{s} / \Sigma_{\text {crit }}\left(z_{d}, z_{s}\right)$ is the dimensionless scaling convergence, $x=\theta /\left(r_{s} / D_{d}\right)$ is the dimensionless angular radius, and $f(x)$ and $g(x)$ are dimensionless functions. We have analytic expressions for $f(x)$ and $g(x)$ as (Bartelmann 1996):

$$
\begin{gathered}
f(x)= \begin{cases}\frac{1}{1-x^{2}}\left(-1+\frac{2}{\sqrt{1-x^{2}}} \operatorname{arctanh} \sqrt{\frac{1-x}{1+x}}\right) & (x<1), \\
\frac{1}{3} & (x=1), \\
\frac{1}{x^{2}-1}\left(+1-\frac{2}{\sqrt{x^{2}-1}} \arctan \sqrt{\frac{x-1}{x+1}}\right) & (x>1),\end{cases} \\
g(x)=\ln \left(\frac{x}{2}\right)+ \begin{cases}\frac{2}{\sqrt{1-x^{2}}} \operatorname{arctanh} \sqrt{\frac{1-x}{1+x}} & (x<1), \\
\frac{2}{\sqrt{x^{2}-1}} \arctan \sqrt{\frac{x-1}{x+1}} & (x>1) .\end{cases}
\end{gathered}
$$


The tangential shear $\gamma_{+, \mathrm{NFW}}(\theta)$ is then evaluated by

$$
\gamma_{+, \mathrm{NFW}}(\theta)=\bar{\kappa}_{\mathrm{NFW}}(\theta)-\kappa_{\mathrm{NFW}}(\theta) .
$$

For a given source redshift $z_{s}$, the Einstein radius is then readily calculated by $1=\kappa_{\mathrm{NFW}}(\theta)$; or more explicitly, using equation (B8) we have

$$
\theta_{\mathrm{E}}^{2}=b \theta_{s}^{2} g\left(\theta_{\mathrm{E}} / \theta_{s}\right)
$$

where $\theta_{s} \equiv r_{s} / D_{d}=r_{\mathrm{vir}} /\left(c_{\mathrm{vir}} D_{d}\right)$ is the angular size of the NFW scale radius. This equation for $\theta_{\mathrm{E}}$ can be solved numerically, for example, by the Newton-Raphson method.

\section{REFERENCES}

Andersson, K. E., \& Madejski, G. M. 2004, ApJ, 607, 190

Baltz, E. A., Marshall, P., \& Oguri, M. 2007, preprint (arXiv: 0705.0682)

Bardeau, S., Soucail, G., Kneib, J.-P., Czoske, O., Ebeling, H., Hudelot, P., Smail, I., \& Smith, G. P. 2007, A\&A, 470, 449

Bardeau, S., et al. 2005, A\&A, 434, 433

Bartelmann, M. 1996, A\&A, 313, 697

Bartelmann, M., Narayan, R., Sfitz, S., \& Schneider, P. 1996, ApJ, 464, L115

Bartelmann, M., \& Schneider, P. 2001, Phys. Rep., 340, 291

Bertin, E., \& Arnouts, S. 1996, A\&AS, 117, 393

Bradač, M., et al. 2006, ApJ, 652, 937

Bridle, S. L., Hobson, M. P., Lasenby, A. N., \& Saunders, R. 1998, MNRAS, 299, 895

Broadhurst, T., \& Barkana, R. 2008, MNRAS, submitted (arXiv: 0801.1875)

Broadhurst, T., Takada, M., Umetsu, K., Kong, X., Arimoto, N., Chiba, M., \& Futamase, T. 2005a, ApJ, 619, L143 (B05a)

Broadhurst, T., Taylor, A. N., \& Peacock, J. A. 1995, ApJ, 438, 49

Broadhurst, T., Umetsu, K., Medezinski, E., Oguri, M., \& Rephaeli, Y. 2008, ApJ, submitted (arXiv: 0805.2617)

Broadhurst, T., et al. 2005b, ApJ, 621, 53 (B05b)

Bullock, J. S., Kolatt, T. S., Sigad, Y., Somerville, R. S., Kravtsov, A. V., Klypin, A. A., Primack, J. R., \& Dekel, A. 2001, MNRAS, 321, 559

Capak, P., et al. 2004, AJ, 127, 180

2007, ApJS, 172, 99

Clowe, D. 2003, in ASP Conf. Ser. 301, Matter and Energy in Clusters of Galaxies, ed. S. Bowyer \& C.-Y. Hwang (San Francisco: ASP), 271

Clowe, D., Luppino, G. A., Kaiser, N., \& Gioia, I. M. 2000, ApJ, 539, 540

Cobb, B. E., Bailyn, C. D., van Dokkum, P. G., \& Natarajan, P. 2006, ApJ, 651, L85

Comerford, J. M., \& Natarajan, P. 2007, MNRAS, 379, 190

Corless, V. L., \& King, L. J. 2007, MNRAS, 380, 149

Crittenden, R. G., Natarajan, P., Pen, U.-L., \& Theuns, T. 2002, ApJ, 568, 20 Czoske, O. 2004, in IAU Colloq. 195, Outskirts of Galaxy Clusters: Intense

Life in the Suburbs, ed. A. Diaferio (Cambridge: Cambridge Univ. Press), 18

Eke, V. R., Cole, S., \& Frenk, C. S. 1996, MNRAS, 282, 263

Erben, T., Van Waerbeke, L., Bertin, E., Mellier, Y., \& Schneider, P. 2001, A\&A, 366, 717

Fahlman, G., Kaiser, N., Squires, G., \& Woods, D. 1994, ApJ, 437, 56

Gavazzi, R., Fort, B., Mellier, Y., Pello, R., \& Dantel-Fort, M. 2003, A\&A, 403, 11

Girardi, M., Fadda, D., Escalera, E., Giuricin, G., Mardirossian, F., \& Mezzetti, M. 1997, ApJ, 490, 56

Grossi, M., Dolag, K., Branchini, E., Matarrese, S., \& Moscardini, L. 2007, MNRAS, 382, 1261

Halkola, A. Seitz, S., \& Pannella, M. 2006, MNRAS, 372, 1425

Hennawi, J. F., Dalal, N., Bode, P., \& Ostriker, J. P. 2007, ApJ, 654, 714

Heymans, C., et al. 2006, MNRAS, 368, 1323

Hilbert, S., White, S. D. M., Hartlap, J., \& Schneider, P. 2007, MNRAS, 382, 121

Hobson, M. P., Lasenby, A. N. 1998, MNRAS, 298, 905

Hoekstra, H., Franx, M., Kuijken, K., \& Squires, G. 1998, ApJ, 504, 636

Hudson, M. J., Gwyn, S. D. J., Dahle, H., \& Kaiser, N. 1998, ApJ, 503, 531

Jain, B., Seljak, U., \& White, S. 2000, ApJ, 530, 547

Jing, Y. P., \& Suto, Y. 2000, ApJ, 529, L69

2002, ApJ, 574, 538

Johnston, D. E., et al. 2007, preprint (arXiv: 0709.1159)

Kaiser, N. 1995, ApJ, 439, L1

Kaiser, N., \& Squires, G. 1993, ApJ, 404, 441

Kaiser, N., Squires, G., \& Broadhurst, T. 1995, ApJ, 449, 460
Kawahara, H., Suto, Y., Kitayama, T., Sasaki, S., Shimizu, M., Rasia, E., \& Dolag, K. 2007, ApJ, 659, 257

King, L., \& Corless, V. 2007, MNRAS, 374, L37

King, L. J., Clowe, D. I., \& Schneider, P. 2002, A\&A, 383, 118

Kneib, J.-P., et al. 2003, ApJ, 598, 804

Lemze, D., Barkana, L., Broadhurst, T., \& Rephaeli, Y. 2008, MNRAS, 386, 1092

Leonard, A., Goldberg, D. M., Haaga, J. L., \& Massey, R. 2007, ApJ, 666, 51 Limousin, M., et al. 2007, ApJ, 668, 643

Marshall, P. J., Hobson, M. P., Gull, S. F., \& Bridle, S. L. 2002, MNRAS, 335, 1037

Massey, R., et al. 2007, MNRAS, 376, 13

Maisinger, K., Hobson, M. P., \& Lasenby, A. N. 1997, MNRAS, 290, 313

Medezinski, E., Broadhurst, T., Umetsu, K., Coe, D., Benitez, N., Ford, H., Rephaeli, Y., Arimoto, N., \& Kong, X. 2007, ApJ, 663, 717

Navarro, J. F., Frenk, C. S., White, S. D. M. 1997, ApJ, 490, 493

Neto, A. F., et al. 2007, MNRAS, 381, 1450

Oguri, M., Takada, M., Umetsu, K., \& Broadhurst, T. 2005, ApJ, 632, 841

Oguri, M., Taruya, A., \& Suto, Y. 2001, ApJ, 559, 572

Okabe, N., \& Umetsu, K. 2008, PASJ, 60, 345

Okura, Y., Umetsu, K., \& Futamase, T. 2008, ApJ, 680, 1

Peebles, P. J. E. 1980, The Large-Scale Structure of the Universe (Princeton: Princeton Univ. Press)

Press, W. H., Teukolsky, S., Vetterling, T. W., \& Flannery, B. P. 1992, Numerical Recipes in Fortran (2nd ed.; Cambridge: Cambridge Univ. Press)

Sadeh, S., Rephaeli, Y., \& Silk, J. 2007, MNRAS, 380, 637

Sand, D. J., Treu, T., Smith, G. P., \& Ellis, R. S. 2004, ApJ, 604, 88

Sato, J., Umetsu, K., Futamase, T., \& Yamada, T. 2003, ApJ, 582, L67

Schneider, P., King, L., \& Erben, T. 2000, A\&A, 353, 41

Schneider, P., \& Seitz, C. 1995, A\&A, 294, 411

Seitz, S., Schneider, P., \& Bartelmann, M. 1998, A\&A, 337, 325

Seljak, U. 1998, ApJ, 506, 64

Sereno, M. 2007, MNRAS, 380, 1207

Shimizu, M., Kitayama, T., Sasaki, S., \& Suto, Y. 2003, ApJ, 590, 197

Smith, G. P., et al. 2005, MNRAS, 359, 417

Spergel, D. N., et al. 2003, ApJS, 148, 175

. 2007, ApJS, 170, 377

Springel, V., et al. 2005, Nature, 435, 629

Suyu, S. H., Marshall, P. J., Hobson, M. P., \& Blandford, R. D. 2006, MNRAS, 371, 983

Takada, M., \& Jain, B. 2003, MNRAS, 340, 580

Tasitsiomi, A., et al. 2004, ApJ, 607, 125

Taylor, A. N., Dye, S., Broadhurst, T., Benitez, N., \& van Kampen, E. 1998, ApJ, 501, 539

Taylor, A. N., Kitching, T. D., Bacon, D. J., \& Heavens, A. F. 2007, MNRAS, 374,1377

Teague, P. F., Carter, D., \& Gray, P. M. 1990, ApJS, 72, 715

Tegmark, M., et al. 2004, Phys. Rev. D, 69, 3501

Tyson, J. A., \& Fisher, P. 1995, ApJ, 446, L55

Tyson, J. A., Wenk, R. A., \& Valdes, F. 1990, ApJ, 349, L1

Umetsu, K., Tada, M., \& Futamase, T. 1999, Prog. Theor. Phys. Suppl., 133, 53

Umetsu, K., Takada, M., \& Broadhurst, T. 2007, Mod. Phys. Lett. A, 22, 2099

Wechsler, R. H., Zentner, A. R., Bullock, J. S., Kravtsov, A. V., \& Allgood, B. 2006, ApJ, 652, 71

Wechsler, R. H., et al. 2002, ApJ, 568, 52

Wright, C. O., \& Brainerd, T. G. 2000, ApJ, 534, 34

Zhang, P., \& Pen, U.-L. 2005, Phys. Rev. Lett., 95, 1302 